\title{
ARITHMETIC RESULTS ON ORBITS OF LINEAR GROUPS
}

\author{
MICHAEL GIUDICI, MARTIN W. LIEBECK, CHERYL E. PRAEGER, JAN SAXL, \\ AND PHAM HUU TIEP
}

\begin{abstract}
Let $p$ be a prime and $G$ a subgroup of $G L_{d}(p)$. We define $G$ to be $p$ exceptional if it has order divisible by $p$, but all its orbits on vectors have size coprime to $p$. We obtain a classification of $p$-exceptional linear groups. This has consequences for a well known conjecture in representation theory, and also for a longstanding question concerning $\frac{1}{2}$-transitive linear groups (i.e. those having all orbits on nonzero vectors of equal length), classifying those of order divisible by $p$.
\end{abstract}

\section{INTRODUCTION}

The study of orbits of linear groups acting on finite vector spaces has a long history. Zassenhaus [47] investigated linear groups for which all orbits on nonzero vectors are regular, classifying the insoluble examples, i.e. the insoluble Frobenius complements. If one merely assumes that there is at least one regular orbit, there are many examples and the investigation and classification of these is a lively area of current research. For example, if $p$ is the characteristic and $G$ is a quasisimple irreducible $p^{\prime}$-group, there is almost always a regular orbit, the exceptions being classified in $[19,28]$; this played a major role in the solution of the $k(G V)$-problem [16]. In a different direction, linear groups acting transitively on the set of nonzero vectors were determined by Hering [23], leading to the classification of 2-transitive permutation groups of affine type. Results on groups with few orbits, or a long orbit, or orbits with coprime lengths, can be found in $[11,30,33]$. A much weaker assumption than transitivity is that of $\frac{1}{2}$-transitivity - namely, that all orbits on nonzero vectors have the same size. The soluble linear groups with this property were classified by Passman [38, 39].

In this paper we study linear groups with the following property.

Definition Let $V=V_{d}(p)$ be a vector space of dimension $d$ over $\mathbb{F}_{p}$ with $p$ prime, and let $G \leq G L_{d}(p)=G L(V)$. We say that $G$ is $p$-exceptional if $p$ divides $|G|$ and $G$ has no orbits on $V$ of size divisible by $p$.

Note that if $d=a b$ for positive integers $a, b$, and $q=p^{a}$, then $\Gamma L_{b}(q) \leq G L_{d}(p)$, so the above definition also applies to subgroups of $\Gamma L_{b}(q)$.

If $G \leq G L_{d}(p)$ has a regular orbit on vectors, then $G$ is not $p$-exceptional. On the other hand, if $G$ is transitive (or $\frac{1}{2}$-transitive) on nonzero vectors and has order divisible by $p$, then $G$ is $p$-exceptional.

We shall obtain a classification of all $p$-exceptional linear groups, up to some undecided questions in the imprimitive case. We also give applications to $\frac{1}{2}$-transitive groups, and to a conjecture in representation theory.

We begin with our result for primitive linear groups. In the statement, by the deleted permutation module over $\mathbb{F}_{p}$ ( $p$ prime) for a symmetric group $\mathrm{S}_{c}$, we mean the irreducible $\mathbb{F}_{p} S_{c}$-module $S / S \cap T$, where $S=\left\{\left(a_{1}, \ldots, a_{c}\right): a_{i} \in \mathbb{F}_{p}, \sum a_{i}=0\right\}$ and $T=\{(a, \ldots, a)$ : $\left.a \in \mathbb{F}_{p}\right\}$, and $\mathrm{S}_{c}$ acts by permuting coordinates in the obvious way. Denote by $V^{\sharp}$ the set of nonzero vectors in a vector space $V$. 
Theorem 1. Let $G$ be an irreducible p-exceptional subgroup of $G L_{d}(p)=G L(V)$, and suppose $G$ acts primitively on $V$. Then one of the following holds:

(i) $G$ is transitive on $V^{\sharp}$ (a list can be found in [30, Appendix 1]);

(ii) $G \leq \Gamma L_{1}(q)\left(q=p^{d}\right)$, determined in Lemma 2.7;

(iii) $G$ is one of the following:
(a) $G=\mathrm{A}_{c}, \mathrm{~S}_{c}$ with $c=2^{r}-2$ or $2^{r}-1$, with $V$ the deleted permutation module over $\mathbb{F}_{2}$, of dimension $c-2$ or $c-1$ respectively (see Lemma 9.4);
(b) $S L_{2}(5) \triangleleft G<\Gamma L_{2}(9)<G L_{4}(3)$, orbit sizes $1,40,40$;
(c) $L_{2}(11) \triangleleft G<G L_{5}(3)$, orbit sizes $1,22,110,110$;
(d) $M_{11} \triangleleft G<G L_{5}(3)$, orbit sizes $1,22,220$;
(e) $M_{23}=G<G L_{11}(2)$, orbit sizes $1,23,253,1771$.

For the imprimitive case we first require a result on permutation groups. For a prime $p$, we say a subgroup $K \leq \mathrm{S}_{n}$ is $p$-concealed if it has order divisible by $p$, and all its orbits on the power set of $\{1, \ldots, n\}$ have size coprime to $p$. The following result is an extension of $[5,41]$, which classify primitive groups having no regular orbit on the power set.

Theorem 2. Let $H$ be a primitive subgroup of $\mathrm{S}_{n}$ of order divisible by a prime $p$. Then $H$ is $p$-concealed if and only if one of the following holds:

(i) $\mathrm{A}_{n} \triangleleft H \leq \mathrm{S}_{n}$, and $n=a p^{s}-1$ with $s \geq 1, a \leq p-1$ and $(a, s) \neq(1,1)$; also $H \neq \mathrm{A}_{3}$ if $(n, p)=(3,2)$;

(ii) $(n, p)=(8,3)$, and $H=A G L_{3}(2)=2^{3}: S L_{3}(2)$ or $H=A \Gamma L_{1}(8)=2^{3}: 7: 3$;

(iii) $(n, p)=(5,2)$ and $H=D_{10}$, a dihedral group of order 10 .

Here is our main result on imprimitive $p$-exceptional linear groups.

Theorem 3. Suppose $G \leq G L_{d}(p)=G L(V)$ is irreducible, $p$-exceptional and imprimitive, and also $G=O^{p^{\prime}}(G)$. Let $V=V_{1} \oplus \ldots \oplus V_{n}(n>1)$ be any imprimitivity decomposition for $G$. Then $\operatorname{Stab}_{G}\left(V_{1}\right)$ is transitive on $V_{1}^{\sharp}$, and $G$ induces a primitive p-concealed subgroup of $\mathrm{S}_{n}$ on $\left\{V_{1}, \ldots, V_{n}\right\}$.

There is a partial converse: if $X \leq G L\left(V_{1}\right)$ is transitive on $V_{1}^{\sharp}$ and $H \leq \mathrm{S}_{n}$ is primitive and $p$-concealed, then the full wreath product $X \imath H$ acting on $V=V_{1}^{n}$ is $p$-exceptional (see Lemma 2.5).

The following is a general structure theorem for irreducible $p$-exceptional groups.

Theorem 4. Let $G \leq G L_{d}(p)=G L(V)$ be an irreducible p-exceptional group, and let $G_{0}=O^{p^{\prime}}(G)$. Write $V \downarrow G_{0}=V_{1} \oplus \cdots \oplus V_{t}$ with $V_{i}$ irreducible $G_{0}$-modules. Then $G_{0}^{V_{1}}$ is either a primitive p-exceptional group (given by Theorem 1), or an imprimitive p-exceptional group (given by Theorem 3). Moreover, the $V_{i}$ are pairwise non-isomorphic $G_{0}$-modules, and $G$ acts on $\left\{V_{1}, \ldots, V_{t}\right\}$ as a transitive $p^{\prime}$-group.

Again, there is a partial converse (Lemma 2.5): the full wreath product of a $p$-exceptional group and a transitive $p^{\prime}$-group is $p$-exceptional.

The next result has important applications in the modular representation theory of finite groups. Recall that, if $p$ is any prime and $B$ is a Brauer $p$-block of any finite group $G$ with defect group $P$, then the Brauer height zero conjecture asserts that all irreducible complex characters in $B$ have height zero if and only if $P$ is abelian. One of the significant results of the representation theory of finite groups in the 1980's was to prove that if $G$ is $p$-soluble and $\lambda \in \operatorname{Irr}(Z)$ is an irreducible complex character of a normal subgroup $Z \triangleleft G$ such that $\chi(1) / \lambda(1)$ is not divisible by $p$ for all $\chi \in \operatorname{Irr}(G)$ lying over $\lambda$, then $G / Z$ has abelian Sylow $p$-subgroups. This theorem, established by D. Gluck and T. Wolf in $[17,18]$ led to a proof of the Brauer height zero conjecture for $p$-soluble groups. As shown by very recent results on the Brauer height zero conjecture, in particular, the proof [36] of the conjecture in the case $p=2$ and $P \in \operatorname{Syl}_{p}(G)$, as well as reduction theorems for the conjecture [34] and [35], one of the main obstacles to proving the conjecture in full generality is to obtain a proof of the Gluck-Wolf theorem for arbitrary finite groups. This has now been achieved in [37], which uses Theorem 5 in a crucial way. 
Theorem 5. Let $G$ be a non-identity finite group and let $p$ be an odd prime. Assume that $G=O^{p^{\prime}}(G)=O^{p}(G)$ and $G$ has abelian Sylow p-subgroups. Suppose that $V$ is a finitedimensional, faithful, irreducible $\mathbb{F}_{p} G$-module such that every orbit of $G$ on $V$ has length coprime to $p$. Then one of the following holds:

(i) $G=S L_{2}(q)$ and $|V|=q^{2}$ for some $q=p^{a}$;

(ii) $G$ acts transitively on the $n$ summands of a decomposition $V=\bigoplus_{i=1}^{n} V_{i}$, where $p<n<p^{2}, n \equiv-1 \bmod p$. Furthermore, $\operatorname{Stab}_{G}\left(V_{1}\right)$ acts transitively on $V_{1}^{\sharp}$, and the action of $G$ on $\left\{V_{1}, \ldots, V_{n}\right\}$ induces either $\mathrm{A}_{n}$, or the affine group $2^{3}: S L_{3}(2)$ for $(n, p)=(8,3)$;

(iii) $(G,|V|)=\left(S L_{2}(5), 3^{4}\right),\left(2_{-}^{1+4} \cdot \mathrm{A}_{5}, 3^{4}\right),\left(L_{2}(11), 3^{5}\right),\left(M_{11}, 3^{5}\right)$, or $\left(S L_{2}(13), 3^{6}\right)$.

Here is a further consequence concerning $\frac{1}{2}$-transitive linear groups.

Theorem 6. Let $G \leq G L_{d}(p)$ (p prime) be a $\frac{1}{2}$-transitive linear group, and suppose $p$ divides $|G|$. Then one of the following holds:

(i) $G$ is transitive on the set of nonzero vectors (given by [30, Appendix]);

(ii) $G \leq \Gamma L_{1}\left(p^{d}\right)$;

(iii) $S L_{2}(5) \triangleleft G \leq \Gamma L_{2}(9)<G L_{4}(3)$ and $G$ has two orbits on nonzero vectors of size 40 .

Concerning part (ii) of the theorem, some examples of $\frac{1}{2}$-transitive subgroups of $\Gamma L_{1}\left(p^{d}\right)$ are given in Lemma 2.7 .

Recall that a finite transitive permutation group is said to be $\frac{3}{2}$-transitive if all nontrivial orbits of a point stabiliser have the same size, this size being greater than 1 . By [45, Theorem 10.4], such groups are either Frobenius groups or primitive. Steps towards the classification of the primitive examples were taken in [2], where it was shown that they must be either almost simple or affine, and the former were classified. The next result deals with the modular affine case. The non-modular case will be the subject of a future paper.

Corollary 7. If $G \leq A G L_{d}(p)$ is a $\frac{3}{2}$-transitive affine permutation group with pointstabiliser $G_{0}$ of order divisible by $p$, then one of the following holds:

(i) $G$ is 2-transitive;

(ii) $G \leq A \Gamma L_{1}\left(p^{d}\right)$;

(iii) $S L_{2}(5) \triangleleft G_{0} \leq \Gamma L_{2}(9)<G L_{4}(3)$ and $G$ has rank 3 with subdegrees $1,40,40$.

The layout of the paper is as follows. Theorems 2 and 3 are proved in Section 3 ; then the proof of Theorem 1 is given in the next nine sections, culminating in Section 12. The deductions of Theorems 4, 5 and 6 can be found in the final Section 13 .

Notation: The following notation will be used throughout the paper. For a vector space $V$, an element $g \in G L(V)$ and a subgroup $H \leq G L(V)$,

$$
\begin{aligned}
& V^{\sharp}=V \backslash\{0\} \\
& C_{V}(g)=\{v \in V: v g=v\} \\
& C_{V}(H)=\{v \in V: v h=v \text { for all } h \in H\} \\
& V \downarrow H=\text { restriction of } V \text { to } H .
\end{aligned}
$$

Also $x^{G}$ denotes the conjugacy class of an element $x$ in a group $G$, and $J_{k}$ denotes a unipotent Jordan block of size $k$.

Acknowledgements The first four authors acknowledge the support of an ARC Discovery Grant; Giudici acknowledges the support of an Australian Research Fellowship; Praeger acknowledges a support of a Federation Fellowship; Tiep acknowledges the support of the NSF (grant DMS-0901241).

\section{Preliminaries}

We begin with a simple observation. 
Lemma 2.1. Let $H \leq G L_{d}(p)$ be p-exceptional on $V=\mathbb{F}_{p}^{d}$.

(i) If $K$ is a normal subgroup of $H$ and $p$ divides $|K|$, then $K$ is $p$-exceptional on $V$.

(ii) If $N \leq G L_{d}(p)$ has order coprime to $p$ and $N$ is normalised by $H$, then $N H$ is $p$-exceptional on $V$.

Proof (i) If $K$ had an orbit, say $\Delta$, in $V$ of length a multiple of $p$, then the $H$-orbit containing $\Delta$ would have length divisible by $|\Delta|$ since $K$ is normal in $H$, contradicting the fact that $H$ is $p$-exceptional.

(ii) Let $L:=N H$, let $v \in V^{\sharp}$, and consider $v^{L}$ and $v^{N}$, the $L$-orbit and $N$-orbit containing $v$, respectively. Since $N$ is normal in $L, v^{L}$ is the set theoretic union of a subset $\mathcal{B}_{0}$ of the set $\mathcal{B}$ of $N$-orbits in $V$, and $\mathcal{B}_{0}$ is an $H$-orbit in its induced action on $\mathcal{B}$. Moreover $v^{N} \in \mathcal{B}_{0}$ and $\left|v^{L}\right|=\left|v^{N}\right| .\left|\mathcal{B}_{0}\right|$. As $|N|$ is coprime to $p$, also $\left|v^{N}\right|$ is coprime to $p$. Since $H$ acts on $\mathcal{B}$, the $H$-orbit $v^{H}$ consists of a constant number of vectors from each $N$-orbit in $\mathcal{B}_{0}$. Thus $\left|\mathcal{B}_{0}\right|$ divides $\left|v^{H}\right|$ and hence $\left|\mathcal{B}_{0}\right|$ is coprime to $p$.

Lemma 2.2. Let $q=p^{a}$ with $p$ prime, let $Z=Z\left(G L_{n}(q)\right)$ and let $H$ be a subgroup of $\Gamma L_{n}(q)$. Then $H$ is p-exceptional if and only if $Z H$ is $p$-exceptional.

Proof If $Z H$ is $p$-exceptional then so is $H$, by Lemma 2.1(i). The converse follows from Lemma 2.1(ii).

Lemma 2.3. Let $G \leq \Gamma L(V)=\Gamma L_{n}(q)\left(q=p^{a}\right)$ be $p$-exceptional, and let $G_{0}=G \cap G L(V)$. Then one of the following holds:

(i) $p$ divides $\left|G_{0}\right|$ and $G_{0}$ is p-exceptional;

(ii) $G_{0}$ is a $p^{\prime}$-group, and $G$ contains a p-exceptional normal subgroup of the form $G_{0}\langle\sigma\rangle$, where $\sigma \in \Gamma L(V) \backslash G L(V)$ is a field automorphism of order $p$.

Proof If $p$ divides $\left|G_{0}\right|$ then $G_{0}$ is $p$-exceptional by Lemma 2.1, so (i) holds. Now assume $p$ does not divide $\left|G_{0}\right|$. As $G / G_{0}$ is cyclic, we have $G=G_{0}\langle x\rangle$ for some $x$ of order divisible by $p$. Taking $\sigma$ to be a power of $x$ of order $p$, we obtain (ii).

The next lemma will be used many times in the proof of Theorem 1.

Lemma 2.4. Let $G \leq G L(V)=G L_{d}(q)$ with $q=p^{a}$ (p prime), and suppose that $G$ is $p$ exceptional and $C_{V}\left(O^{p^{\prime}}(G)\right)=0$. Let $t$ be an element of $G$ of order $p$, and let $P \in S y l_{p}(G)$.

(i) Then $d=\operatorname{dim} V \leq r_{p} \log _{q}\left|G: N_{G}(P)\right|$, where $r_{p}$ is the minimal number of conjugates of $P$ generating $O^{p^{\prime}}(G)$.

(ii) We have $|V| \leq\left|C_{V}(t)\right| \cdot\left|t^{G}\right|$.

(iii) Suppose $O^{p^{\prime}}(G)$ is generated by $\alpha$ conjugates of $t$. Then $q^{d / \alpha} \leq\left|t^{G}\right|$.

Proof As $G$ is $p$-exceptional, every nonzero vector is fixed by some conjugate of $P$, so

$$
V=\bigcup_{g \in G} C_{V}\left(P^{g}\right) .
$$

Moreover, $\operatorname{dim} C_{V}(P) \leq d\left(1-\frac{1}{r_{p}}\right)$, since otherwise the group generated by $r_{p}$ conjugates of $P$ would have a nonzero centralizer in $V$, contrary to the hypothesis. Hence

$$
q^{d}=|V| \leq\left|G: N_{G}(P)\right| q^{d\left(1-\frac{1}{r_{p}}\right)} .
$$

This gives (i).

For (ii), observe that every nonzero vector in $V$ is fixed by a conjugate of $t$ (as $G$ is $p$ exceptional), so $V=\bigcup_{g \in G} C_{V}\left(t^{g}\right)$, which implies (ii). Finally, (iii) follows from (ii) together with the fact that $\operatorname{dim} C_{V}(t) \leq d\left(1-\frac{1}{\alpha}\right)$ (which follows from the argument of the first paragraph).

The next lemma proves the existence of many examples of imprimitive $p$-exceptional linear groups, giving partial converse statements to Theorems 3 and 4 . 
Lemma 2.5. Let $V_{1}=\mathbb{F}_{p}^{k}$, let $n$ be a positive integer, and let $V=V_{1}^{n}=\mathbb{F}_{p}^{k n}$. Suppose $G_{1} \leq G L\left(V_{1}\right)$ and $H \leq \mathrm{S}_{n}$ are such that one of the following conditions holds:

(i) $G_{1}$ is transitive on $V_{1}^{\sharp}$ and $H$ is p-concealed,

(ii) $G_{1}$ is p-exceptional and $H$ is a $p^{\prime}$-group.

Then the wreath product $G=G_{1}$ wr $H$, acting naturally on $V$, is p-exceptional.

Proof Suppose (i) holds, and let $0 \neq v=\left(v_{1}, \ldots, v_{n}\right) \in V_{1}^{n}=V$. Let $i_{1}, \ldots, i_{k}$ be the positions $i$ for which $v_{i} \neq 0$. Then the orbit $v^{G}$ has size $\left|V_{1}^{\sharp}\right|^{k} \cdot \delta$, where $\delta$ is the size of the orbit of $H$ on $k$-sets containing $\left\{i_{1}, \ldots, i_{k}\right\}$. As $H$ is $p$-concealed, $p$ does not divide $\delta$, and so $\left|v^{G}\right|$ is coprime to $p$. The argument for (ii) is similar.

We shall need the following upper bounds on the order of $p^{\prime}$-subgroups of $G L_{m}(q)$ for $m=2,3$.

Lemma 2.6. Let $q=p^{f} \geq 4$ and let $A$ be a $p^{\prime}$-subgroup of $G L_{m}(q)$.

(i) If $m=2$ and $q \neq 5,7,11$ or 19 , then $|A| \leq\left(q^{2}-1\right) \cdot(2, q-1)$.

(ii) If $m=3$ then $|A| \leq(q-1)\left(q^{3}-1\right)$.

Proof (i) It suffices to show that any $p^{\prime}$-subgroup of $P G L_{2}(q)(q \neq 5,7,11,19)$ has order at most $(q+1) \cdot(2, q-1)$. From the list of subgroups of $P G L_{2}(q)$ in [10, Chapter XII], any subgroup of order at least $(q+1) \cdot(2, q-1)$ has order dividing one of $2(q+1), q(q-1), 24$ (if $q=4$ or 9 ), or 60 (if $q=4,9$ or 29 ). The assertion follows.

(ii) The bound can be checked directly using [8] for $q \leq 11$, so we will assume $q \geq 13$. If $A$ is reducible on $\mathbb{F}_{q}^{3}$, then $A$ is contained in a maximal parabolic subgroup $P$ of $G L_{3}(q)$, and so $|A| \leq|P|_{p^{\prime}}=\left(q^{2}-1\right)(q-1)^{2}$. If $A$ is irreducible but imprimitive, then $|A| \leq 6(q-1)^{3}$. Finally, if $A$ is irreducible and primitive, then $|A| \leq q^{3} \cdot \log _{2} q^{3}$ by the main result of [14]. In all cases $|A|<(q-1)\left(q^{3}-1\right)$ since $q \geq 13$.

Now we consider the case of $p$-exceptional 1-dimensional semilinear groups. Here we identify $V=\mathbb{F}_{p}^{d}$ with the field $\mathbb{F}_{p^{d}}$. Let $\omega$ be a primitive element of $\mathbb{F}_{p^{d}}$ and let $\varphi: x \rightarrow x^{p}$ denote the Frobenius automorphism. The 1-dimensional semilinear groups are subgroups of $\Gamma L_{1}\left(p^{d}\right)=\langle\hat{\omega}, \varphi\rangle$, where $\hat{\omega}$ denotes the multiplication map $x \mapsto x \omega$. We determine all such $p$-exceptional groups and show that, when $p$ divides $d$, there exists a unique minimal example.

Lemma 2.7. Suppose that $H \leq \Gamma L_{1}\left(p^{d}\right)$ and $H$ is $p$-exceptional on $V=\mathbb{F}_{p^{d}}$. Then $p$ divides $d$ and there is a factorisation $d=p^{k} s$ for some $k \geq 1$ such that $H$ has a normal subgroup $K=\left\langle\hat{\omega}^{\left(p^{s}-1\right) / j}, \varphi^{s}\right\rangle$ of index coprime to $p$, for some $j$ dividing $p^{s}-1$. Moreover all such subgroups $H$ and $K$ are p-exceptional and each contains the p-exceptional group $\left\langle\hat{\omega}^{p^{d / p}-1}, \varphi^{d / p}\right\rangle$. The group $K$ is $\frac{1}{2}$-transitive on $V^{\sharp}$, having $\frac{p^{s}-1}{j}$ orbits of length $\frac{p^{d}-1}{p^{s}-1} \cdot j$.

Proof Write $H_{0}=H \cap\langle\hat{\omega}\rangle=\left\langle\hat{\omega}^{c}\right\rangle$, say, where $c$ divides $p^{d}-1$. Then $\left|H_{0}\right|=\left(p^{d}-1\right) / c$ is coprime to $p$, and $H / H_{0} \cong H\langle\hat{\omega}\rangle /\langle\hat{\omega}\rangle$ is isomorphic to a subgroup of $\langle\varphi\rangle$ and hence is cyclic of order dividing $d$. Since $p$ divides $|H|$ it follows that $p$ divides $d$. Let $d=p^{k} s$ where $p^{k}$ is the $p$-part of $|H|$. Then $H$ has a unique normal subgroup $K$ containing $H_{0}$ such that $\left|K / H_{0}\right|=p^{k}$. The group $K$ is generated by $\hat{\omega}^{c}$ and some element $\tau$ of the form $\varphi^{s} \hat{\omega}^{b}$. We may assume that $|\tau|=p^{k}$. A routine computation shows that $\tau^{p^{k}}=\hat{\omega}^{b\left(p^{d}-1\right) /\left(p^{s}-1\right)}$, and hence $p^{s}-1$ divides $b$, say $b=\left(p^{s}-1\right) b^{\prime}$.

By Lemma 2.1, $K$ is $p$-exceptional. This implies in particular that $\tau$ fixes setwise each of the $H_{0}$-orbits in $V^{\sharp}$, and these orbits are the multiplicative cosets $\omega^{i}\left\langle\omega^{c}\right\rangle$ for $0 \leq i \leq c-1$. Now $\tau$ maps $\omega^{i}$ to $\omega^{i p^{s}+b}$ and this element must therefore lie in $\omega^{i}\left\langle\omega^{c}\right\rangle$. It follows that $i\left(p^{s}-1\right)+b=\left(i+b^{\prime}\right)\left(p^{s}-1\right)$ is divisible by $c$. Choosing $i$ such that $i+b^{\prime} \equiv 1(\bmod c)$, we conclude that $c$ divides $p^{s}-1$, say $c=\left(p^{s}-1\right) / j$. This means that $\hat{\omega}^{b} \in H_{0}$, and hence that $K=\left\langle\hat{\omega}^{\left(p^{s}-1\right) / j}, \varphi^{s}\right\rangle$. 
The computation in the previous paragraph shows that $\varphi^{s}$ fixes each $H_{0}$-orbit setwise and hence that $K$ has $c$ orbits of length $\left(p^{d}-1\right) / c$ on nonzero elements of $V$. In particular $K$ is $p$-exceptional and hence any subgroup $H$ containing $K$ with index coprime to $p$, and intersecting $\langle\hat{\omega}\rangle$ in $H_{0}$ is also $p$-exceptional. Finally each of these subgroups $K$ contains the group $\left\langle\hat{\omega}^{p^{d / p}-1}, \varphi^{d / p}\right\rangle$, and our arguments (with $k=1$ ) show that this group is $p$-exceptional.

Next we analyse the possibilities for 2-dimensional semilinear $p$-exceptional groups. We use the following notation: $Z$ denotes the group of scalar matrices in $G L_{2}\left(p^{f}\right)$; the group of diagonal matrices is denoted by $T$; and $\varphi$ denotes the Frobenius map $\left(a_{i j}\right) \mapsto\left(a_{i j}^{p}\right)$.

Lemma 2.8. Suppose that $H \leq \Gamma L_{2}\left(p^{f}\right)$ and $H$ is $p$-exceptional on $V=\mathbb{F}_{p^{f}}^{2}$. Then one of the following holds.

(i) $H$ contains $S L_{2}\left(p^{f}\right)$.

(ii) $p$ divides $2 f, H \cap G L_{2}\left(p^{f}\right)$ is contained in $\left\langle T,\left(\begin{array}{cc}0 & 1 \\ -1 & 0\end{array}\right)\right\rangle$ if $p$ is odd, or $T$ if $p=2$.

(iii) $p$ divides $2 f$, and $H \leq \Gamma L_{1}\left(p^{2 f}\right)$ is as in Lemma 2.7.

(iv) $p^{f}=9$ and $S L_{2}(5) \triangleleft H \cap G L_{2}(9)$.

Proof If $H$ contains $S L_{2}\left(p^{f}\right)$ then $H$ is transitive on $V^{\sharp}$ so $H$ is $p$-exceptional. Suppose now that this is not the case, and let $H_{0}=H \cap G L_{2}\left(p^{f}\right)$.

Observe that, for a proper subfield $\mathbb{F}_{p^{c}}$ of $\mathbb{F}_{p^{f}}$ the group $S L_{2}\left(p^{c}\right)$ acts regularly on the orbit containing $(1, \omega)$ where $\omega$ is a primitive element of $\mathbb{F}_{p^{f}}$. If $S L_{2}\left(p^{c}\right)$ were normal in $H$ then the $H$-orbit containing $(1, \omega)$ would have length a multiple of $p$. Thus $H$ has no normal subgroup conjugate to $S L_{2}\left(p^{c}\right)$ for any proper divisor $c$ of $f$. Moreover if $f \geq 3 c$, then the stabiliser in $Z \circ S L_{2}\left(p^{c}\right)$ of the 1 -space $\langle(1, \omega)\rangle$ is $Z$. Hence, if $p$ divides $\left|H_{0}\right|$ then $H_{0}$ is not contained in a conjugate of $Z \circ S L_{2}\left(p^{c}\right)$ for any divisor $c$ of $f$ such that $f \geq 3 c$.

Observe that the $T$-orbits in $V$ have lengths $1, q-1, q-1,(q-1)^{2}$. Thus if $p$ divides $f$ then any subgroup of $T \cdot\langle\varphi\rangle$ containing $T$ and of order divisible by $p$ is $p$-exceptional. If $p$ is odd the same is true for such subgroups of T.2. $\langle\varphi\rangle$. These examples and some of their subgroups are listed in (ii). So suppose that $H_{0}$ is not conjugate to a subgroup of T.2.

Also if $H_{0}$ preserves on $V$ the structure of a 1-dimensional space over $\mathbb{F}_{p^{2 f}}$, then $H$ is a 1-dimensional semilinear group and we obtain the examples in (iii) by Lemma 2.7.

If $H_{0}$ has a non-trivial normal $p$-subgroup $K$ then for a vector $v$ not fixed by $K$, the $H$-orbit containing $v$ is a union of some $K$-orbits, each of length a nontrivial power of $p$. Thus no such subgroup exists.

Consider $\bar{H}_{0} \cong H_{0} Z / Z \leq P G L_{2}\left(p^{f}\right)$. From our arguments so far, and the classification of subgroups of $P G L_{2}\left(p^{f}\right)$ [10, Chapter XII], we may assume that $\bar{H}_{0} \cong \mathrm{A}_{4}, \mathrm{~S}_{4}$ (with $p$ odd) or $\mathrm{A}_{5}$ (with $\left.p^{f} \equiv \pm 1 \bmod 10\right)$, and that $H_{0}$ is not realisable modulo scalars over a proper subfield $\mathbb{F}_{p^{c}}$ with $f \geq 3 c$. In particular then, $p$ is odd and $f$ divides 4 . Thus $p$ divides $\left|H_{0}\right|$ and hence $p=3\left(\right.$ as $p \neq 5$ if $\left.\bar{H}_{0}=\mathrm{A}_{5}\right)$. If $\bar{H}_{0}=\mathrm{A}_{4}$ or $\mathrm{S}_{4}$ then $H_{0} \triangleright S L_{2}(3)$ which is not the case, so $\bar{H}_{0}=A_{5}$, and $p^{f}=9$ or 81 . In the latter case one checks that the orbit of $H_{0}$ containing the vector $(1, \omega)$ has size divisible by 3 . Hence $p^{f}=9$, which leads to the examples in (iv) since $Z \circ S L_{2}(5)$ is transitive on the nonzero vectors.

The next two lemmas concern the usual action of a group $G$ on a quotient group $G / V$ defined by $(V x)^{g}=V x^{g}$.

Lemma 2.9. Let $G$ be a finite group, $p$ a prime, and suppose $G$ has a normal subgroup $V$ which is an elementary abelian p-group. If $t \in G$ is a $p^{\prime}$-element, then $C_{G}(t) / C_{V}(t) \cong$ $C_{G / V}(t)$.

Proof Assume first that $[V, t]=V$. Let $g \in G$ be a preimage of an element of $C_{G / V}(t)$, so that $t^{g}=t v$ for some $v \in V$. By assumption there exists $u \in V$ such that $[t, u]=v$. Then $t^{u}=t v=t^{g}$, so $g \in V C_{G}(t)$. This shows that $C_{G / V}(t)=V C_{G}(t) / V$, as required. 
Now consider the general case. Writing $V$ additively, we have $V=[V, t] \oplus C_{V}(t)$. Again let $g \in G$ with $t^{g}=t v, v \in V$, and write $v=v_{1}+v_{2}$ with $v_{1} \in[V, t], v_{2} \in C_{V}(t)$. If $v_{2} \neq 0$ then $t v=t\left(v_{1}+v_{2}\right)$ has order divisible by $p$, a contradiction (as $t$ is a $p^{\prime}$-element). Hence $v_{2}=0$, and now we argue as in the first paragraph of the proof.

Corollary 2.10. Let $G$ be a finite group, $p$ a prime, and suppose $G$ has a normal p-subgroup $V$ such that $V / Z(V)$ is elementary abelian and $Z(V) \leq Z(G)$. If $t \in G$ is a $p^{\prime}$-element, then $C_{G}(t) / C_{V}(t) \cong C_{G / V}(t)$.

Proof Write $\bar{G}=G / Z(V)$. By Lemma 2.9, $\bar{V} C_{\bar{G}}(t) / \bar{V}=C_{\bar{G} / \bar{V}}(t)$. If $g \in G$ is a preimage of an element of $C_{\bar{G}}(t)$ then $t^{g}=t z$ for some $z \in Z(V)$. Since this has $p^{\prime}$ order, we must have $z=1$, and the conclusion follows.

\section{IMPRIMITIVE GROUPS}

In this section we prove Theorems 2 and 3. First, for Theorem 2, we determine the primitive $p$-concealed groups, i.e. primitive subgroups $H$ of $\mathrm{S}_{n}$, such that the prime $p$ divides $|H|$ and every orbit of $H$ on the set of all subsets of $\{1, \ldots, n\}$ has length coprime to $p$.

\section{Proof of Theorem 2}

Let $\Omega=\{1, \ldots, n\}$, and define $\Omega_{k}:=\{X \subseteq \Omega|| X \mid=k\}$ for $0 \leq k \leq n$. Let $H$ be a primitive subgroup of $S_{n}$ of order divisible by a prime $p$.

Assume first that $H \geq \mathrm{A}_{n}$. Since $p$ divides $|H|$, we have $n \geq p$ and $H \neq \mathrm{A}_{3}$ if $(n, p)=$ $(3,2)$, and $H$ has exactly one orbit on $\Omega_{p-1}$. Furthermore, $p$ is coprime to $\left|\Omega_{p-1}\right|$ precisely when $p$ does not divide any of the $p-1$ consecutive integers $n-p+2, n-p+3, \ldots, n$, that is, $p \mid(n+1)$. Now we can write $n=\sum_{i=1}^{s} a_{i} p^{i}-1$, where $s \geq 1, a_{s}>0$, and $p-1 \geq a_{i} \geq 0$. Suppose that $n \neq a_{s} p^{s}-1$. Choosing $k:=p^{s}-1$, we see that

$$
\left\lfloor\frac{n}{p^{s}}\right\rfloor-\left\lfloor\frac{k}{p^{s}}\right\rfloor-\left\lfloor\frac{n-k}{p^{s}}\right\rfloor=a_{s}-0-\left(a_{s}-1\right)=1,
$$

and so $p$ divides $\left|\Omega_{k}\right|$. Next suppose that $n=a_{s} p^{s}-1$. Write any $\ell$ between 0 and $n$ as $\ell=$ $\sum_{i=0}^{s} b_{i} p^{i}$ with $0 \leq b_{i} \leq p-1$. Then $b_{s} \leq a_{s}-1$ and $n-\ell=\left(a_{s}-b_{s}-1\right) p^{s}+\sum_{i=0}^{s-1}\left(p-b_{i}-1\right) p^{i}$. Hence, for $0 \leq r \leq s$,

$\left\lfloor\frac{n}{p^{r}}\right\rfloor-\left\lfloor\frac{\ell}{p^{r}}\right\rfloor-\left\lfloor\frac{n-\ell}{p^{r}}\right\rfloor=\left(a_{s} p^{s-r}-1\right)-\sum_{i=r}^{s} b_{i} p^{i-r}-\left(a_{s}-b_{s}-1\right) p^{s-r}-\sum_{i=r}^{s-1}\left(p-b_{i}-1\right) p^{i-r}=0$,

and so $p$ does not divide $\left|\Omega_{\ell}\right|$. Since $n \geq 3, H$ is transitive on $\Omega_{\ell}$. We have shown that $H$ is $p$-concealed if $n=a_{s} p^{s}-1$.

From now on we will assume that $H \nsupseteq \mathrm{A}_{n}$. Clearly, if $H$ contains a normal subgroup $K$ which is also primitive of order divisible by $p$ and has a regular orbit $\Delta$ on $2^{\Omega}$, then the $H$-orbit containing $\Delta$ has length divisible by $p$. Hence, we may assume that $H$ has no regular orbit on $2^{\Omega}$ and apply [41, Theorem 2] to $H$. In all but three of the cases listed in [41, Theorem 2] for $H$, either we can find a subgroup $K$ with the prescribed properties, or we can use GAP [15] or Magma [4] to show directly that $H$ is not a $p$-concealed group. The three exceptional cases give the examples in parts (ii) and (iii) of Theorem 2.

The main result of this section is the following:

Theorem 3.1. Assume $G<G L(V)$ is a (not necessarily irreducible) p-exceptional group which acts primitively as a permutation group on the $n$ summands of the direct sum decomposition

$$
\mathbb{F}_{q}^{m n}=V=V_{1} \oplus V_{2} \oplus \ldots \oplus V_{n},
$$

where $\operatorname{dim}_{\mathbb{F}_{q}} V_{i}=m \geq 1$ and $n \geq 2$. Let $H \leq \mathrm{S}_{n}$ be the subgroup induced by this primitive action. Then one of the following holds:

(i) $H$ is a $p^{\prime}$-group; 
(ii) One of (i), (ii) and (iii) of Theorem 2 holds for H. Moreover, $\operatorname{Stab}_{G}\left(V_{1}\right)$ is transitive on $V_{1}^{\sharp}$.

Proof Assume that $p$ divides $|H|$. First we show that $H$ is $p$-concealed, and so (i), (ii) or (iii) of Theorem 2 holds for $H$. Indeed, suppose that an $H$-orbit $\Delta$ of $H$ on the subsets of $\Omega:=\left\{V_{1}, \ldots, V_{n}\right\}$ has length divisible by $p$. Pick $X=\left\{V_{j_{1}}, \ldots, V_{j_{t}}\right\} \in \Delta$, $0 \neq v_{i} \in V_{j_{i}}$ for $1 \leq i \leq t$, and let $v:=v_{j_{1}}+\ldots+v_{j_{t}}$. Then $I:=\operatorname{Stab}_{G}(v)$ preserves $X$ and so $I K / K \leq \operatorname{Stab}_{H}(X)$ for $K:=\cap_{r=1}^{n} \operatorname{Stab}_{G}\left(V_{r}\right)$. Since $p$ divides $|\Delta|$, it must divide $[G: I]=\left|v^{G}\right|$, contrary to the $p$-exceptionality of $G$.

Now we assume that $H$ satisfies one of the conclusions of Theorem 2 , but $G_{1}:=\operatorname{Stab} b_{G}\left(V_{1}\right)$ has at least two orbits $u^{G_{1}}$ and $v^{G_{1}}$ on $V_{1}^{\sharp}$. For each $1 \leq i \leq n$, fix some $g_{i} \in G$ such that $V_{1} g_{i}=V_{i}\left(\right.$ and $\left.g_{1}=1\right)$, and set $u_{i}=u g_{i}, v_{i}=v g_{i}$. Choose any non-empty subsets $X, Y \subset \Omega$ with $X \cap Y=\emptyset$. Also consider $w=\sum_{i=1}^{n} w_{i}$, with $w_{i}=u_{i}$ if $V_{i} \in X, w_{i}=v_{i}$ if $V_{i} \in Y$, and $w_{i}=0$ otherwise. Observe that any $h \in \operatorname{Stab}_{G}(w)$ stabilises both $X$ and $Y$. (Indeed, $h$ fixes $X \cup Y$. Assume that $V_{i} h=V_{j} \in Y$ for some $V_{i} \in X$. Comparing the $V_{j}$-component of $w h=w$, we see that $v g_{j}=v_{j}=u_{i} h=u g_{i} h$, and so $u g_{i} h g_{j}^{-1}=v$. But $g_{i} h g_{j}^{-1}$ stabilises $V_{1}$, so we conclude that $v \in u^{G_{1}}$, a contradiction.) Now the $p$-exceptionality of $G$ implies that $p$ does not divide $|H: J|$ for $J:=\operatorname{Stab}_{H}(X, Y)$, the subgroup of $H$ consisting of all elements that stabilise $X$ and stabilise $Y$.

It remains to exhibit a pair $(X, Y)$ such that $p$ divides $|H: J|$ to get the desired contradiction. In the case (i) of Theorem 2 , we choose $X:=\left\{V_{1}\right\}, Y:=\left\{V_{2}, \ldots, V_{p}\right\}$. Then

$$
|H: J|=\left|\mathrm{S}_{n}:\left(\mathrm{S}_{p-1} \times \mathrm{S}_{n-p}\right)\right|=p \cdot \frac{n !}{p ! \cdot(n-p) !}
$$

is divisible by $p$. In the case (ii) of Theorem 2 , we can choose $X:=\{a\}$ and $Y:=\{b, c\}$ for some distinct $a, b, c \in \Omega$, and check that $|H: J|=168$ is divisible by $p=3$. In the case (iii) of Theorem 2, we can choose $X:=\{a\}$ and $Y:=\{b, c\}$ for some distinct $a, b, c \in \Omega$, and check that $|H: J|=10$ is divisible by $p=2$.

\section{Deduction of Theorem 3}

Theorem 3 follows very quickly from the above theorem. Indeed, suppose $G \leq G L(V)$ is irreducible, imprimitive and $p$-exceptional, with $G=O^{p^{\prime}}(G)$. Let $V=W_{1} \oplus \ldots \oplus W_{m}$ be an imprimitivity decomposition for $G$. Coarsen this to a decomposition $V=V_{1} \oplus \ldots \oplus V_{n}$ such that $G$ acts as a primitive permutation group on $\left\{V_{1}, \ldots, V_{n}\right\}$, where $V_{1}$ is, say, $W_{1} \oplus \ldots \oplus W_{k}$. As $G=O^{p^{\prime}}(G)$, conclusion (ii) of Theorem 3.1 holds for the action of $G$ on $\left\{V_{1}, \ldots, V_{n}\right\}$. In particular, $\operatorname{Stab}_{G}\left(V_{1}\right)$ acts transitively on the nonzero vectors of $V_{1}$. But it also permutes $W_{1}, \ldots, W_{k}$, so $k=1$. Theorem 3 follows.

\section{Tensor products I: $\mathcal{C}_{4}$ CASE}

In this section we handle $p$-exceptional groups preserving tensor product decompositions. These correspond to subgroups of groups in class $\mathcal{C}_{4}$ in Aschbacher's classification of maximal subgroups of classical groups [1], hence the title of this (and forthcoming) sections. If $U$ and $W$ are vector spaces over a field $\mathbb{F}_{q}$, then a central product $G L(U) \circ G L(W)$ acts naturally on $V=U \otimes W$. We also denote by $\operatorname{Stab}_{\Gamma L(V)}(U \otimes W)$ the stabiliser of the tensor decomposition, which is a group $(G L(U) \circ G L(W))\langle\sigma\rangle$ where $\sigma$ is a field automorphism fixing both factors. As usual, write $Z$ for the group $\mathbb{F}_{q}^{*}$ of scalars in $G L(V)$.

Theorem 4.1. Let $V$ be a vector space over $\mathbb{F}_{q}$ of characteristic $p$, and write $V=U \otimes W$, a tensor product over $\mathbb{F}_{q}$ with $\operatorname{dim} U, \operatorname{dim} W \geq 2$. Let $H \leq G L(U) \circ G L(W)<G L(V)$ and suppose $H$ is p-exceptional.

Then $p=2, \operatorname{dim} U=\operatorname{dim} W=2$, and $Z H=\left(G L_{1}\left(q^{2}\right) \circ G L_{1}\left(q^{2}\right)\right) .2$, where involutions in $H$ act nontrivially on both factors $G L_{1}\left(q^{2}\right)$. This group is p-exceptional, and acts reducibly on $V$. 
We also need a result for the semilinear case.

Theorem 4.2. Let $V$ be a vector space over $\mathbb{F}_{q^{p}}$ (of characteristic $p$ ), and write $V=U \otimes W$ with $2 \leq \operatorname{dim} U \leq \operatorname{dim} W$. Let $H \leq \operatorname{Stab}_{\Gamma L(V)}(U \otimes W)$, and assume $H$ is p-exceptional and $H \cap G L(V)$ is a $p^{\prime}$-group.

Then $p=\operatorname{dim} U=2$. In particular, $H \cap G L(V)$ is not absolutely irreducible on $V$.

The proofs are given in the following three subsections.

4.1. Some theory of tensor decompositions. First we give some general theory for tensor decompositions $V=U \otimes W$ of a vector space $V=\mathbb{F}_{q}^{n}$, where $q=p^{f}$ for a prime $p$, $a=\operatorname{dim} U \geq 2, b=\operatorname{dim} W \geq 2$, and $n=a b$.

Let $\left\{u_{1}, \ldots, u_{a}\right\}$ be a basis for $U,\left\{w_{1}, \ldots, w_{b}\right\}$ be a basis for $W$, and write elements of $G L(U), G L(W)$ as matrices with respect to these bases respectively. Then $V$ has an associated basis $\mathcal{B}:=\left\{u_{i} \otimes w_{j} \mid 1 \leq i \leq a, 1 \leq j \leq b\right\}$, which we refer to as the standard basis. For elements $u=\sum_{i} a_{i} u_{i} \in U$ and $\bar{w}=\sum_{j} b_{j} w_{j} \in W$ we denote the element $\sum_{i, j}\left(a_{i} b_{j}\right)\left(u_{i} \otimes w_{j}\right)$ of $V$ by $u \otimes w$. A vector $v \in V$ is called simple if it can be expressed as $v=u \otimes w$ for some $u \in U, w \in W$.

The stabiliser $X:=\operatorname{Stab}_{G L(V)}(U \otimes W)$ in $G L(V)$ of this decomposition is a central product of $X:=G L(U) \circ G L(W)$ and we view elements of $X$ as ordered pairs $(A, B) \in$ $G L(U) \times G L(W)$ modulo the normal subgroup $Z_{0}=\left\{\left(\lambda I, \lambda^{-1} I\right) \mid \lambda \in \mathbb{F}_{q}^{*}\right\}$, where $(A, B)$ : $u_{i} \otimes w_{j} \mapsto u_{i} A \otimes w_{j} B$ (extending linearly). The stabiliser $\hat{X}:=\operatorname{Stab}_{\Gamma L(V)}(U \otimes W)$ in $\Gamma L(V)$ is a semidirect product of $X$ and the group $\langle\sigma\rangle$ of field automorphisms, where $\sigma$ : $\sum_{i j} a_{i j} u_{i} \otimes w_{j} \mapsto \sum_{i j} a_{i j}^{p} u_{i} \otimes w_{j}$

For an arbitrary $v \in V$, the weight of $v$ is defined as the minimum number $k$ such that $v$ can be written as a sum of $k$ simple vectors. It is not difficult to prove that elements of $\hat{X}$ map weight $k$ vectors to weight $k$ vectors; in particular, the notion of a simple vector does not depend on the choice of standard basis. Also, the weight of a vector is well defined since any vector can be written as a sum of $n$ simple vectors (each a scalar multiple of an element of $\mathcal{B})$.

For subspaces $U_{0}$ of $U$ and $W_{0}$ of $W$ (not necessarily proper subspaces), $X_{U_{0} \otimes W_{0}}$ consists of all elements $(A, B)$ such that $U_{0}$ is $A$-invariant and $W_{0}$ is $B$-invariant, and $\hat{X}_{U_{0} \otimes W_{0}}$ is generated by $X_{U_{0} \otimes W_{0}}$ and a conjugate of $\sigma$.

Lemma 4.3. Let $v \in V$ of weight $k$, and suppose that $v=\sum_{i=1}^{k} x_{i} \otimes y_{i}$, where the $x_{i} \in$ $U, y_{i} \in W$. Write $U_{0}=\left\langle x_{1}, \ldots, x_{k}\right\rangle$ and $W_{0}=\left\langle y_{1}, \ldots, y_{k}\right\rangle$. Then

(i) $\operatorname{dim}\left(U_{0}\right)=\operatorname{dim}\left(W_{0}\right)=k$, so $k \leq \min \{a, b\}$.

(ii) If also $v=\sum_{i=1}^{k} x_{i}^{\prime} \otimes y_{i}^{\prime}$, where the $x_{i}^{\prime} \in U, y_{i}^{\prime} \in W$, then $\left\langle x_{1}^{\prime}, \ldots, x_{k}^{\prime}\right\rangle=U_{0}$ and $\left\langle y_{1}^{\prime}, \ldots, y_{k}^{\prime}\right\rangle=W_{0}$.

(iii) Let $A, B$ be the matrices representing the linear transformations $A: x_{i} \mapsto x_{i}^{\prime}, B$ : $y_{i} \mapsto y_{i}^{\prime}$ (for all $i$ ) of $U_{0}, W_{0}$ with respect to the bases $\left\{x_{1}, \ldots, x_{k}\right\}$ and $\left\{y_{1}, \ldots, y_{k}\right\}$ respectively, where $x_{i}^{\prime}, y_{i}^{\prime}$ are as in (ii). Then $B=A^{-T} \in G L(k, q)$.

(iv) $\hat{X}_{v} \leq \hat{X}_{U_{0} \otimes W_{0}}$, the group induced by $X_{v}$ on $U_{0} \otimes W_{0}$ is a diagonal subgroup of the group $G L(k, q) \circ G L(k, q)$ induced by $X_{U_{0} \otimes W_{0}}$, consisting of the pairs $\left(A, A^{-T}\right)$ (modulo $Z_{0}$ ) for $A \in G L(k, q)$ (with respect to the bases in (iii)), and $\hat{X}_{v}=\left\langle X_{v}, \sigma^{\prime}\right\rangle$, where $\sigma^{\prime}$ is conjugate to $\sigma$ and induces a generator of the group of field automorphisms of $G L\left(U_{0} \otimes W_{0}\right)$.

Proof Part (i) follows almost from the definition of $k$, since, for example, if $x_{k}=\sum_{i=1}^{k-1} a_{i} x_{i}$, then $v=\sum_{i=1}^{k-1} x_{i} \otimes\left(y_{i}+a_{i} y_{k}\right)$. For the rest of the proof we will assume without loss of generality that $x_{i}=u_{i}$ and $y_{i}=w_{i}$ for $1 \leq i \leq k$.

Suppose also that $v$ has an expression as in part (ii). For $1 \leq i \leq k$, in terms of the basis $\mathcal{B}$ we have $x_{i}^{\prime}=\sum_{j=1}^{a} a_{i j} u_{j}$ and $y_{i}^{\prime}=\sum_{j=1}^{b} b_{i j} w_{j}$. Write $A_{0}=\left(a_{i j}\right) \in \mathrm{M}_{k \times a}(q)$ and 


$$
\begin{aligned}
& B_{0}=\left(b_{i j}\right) \in \mathrm{M}_{k \times b}(q) \text {. Then } \\
& \qquad v=\sum_{i=1}^{k} \sum_{j=1}^{a} \sum_{\ell=1}^{b} a_{i j} b_{i \ell} u_{j} \otimes w_{\ell}=\sum_{j, \ell}\left(\sum_{i=1}^{k} a_{i j} b_{i \ell}\right) u_{j} \otimes w_{\ell}=\sum_{j, \ell}\left(A_{0}^{T} B_{0}\right)_{j, \ell} u_{j} \otimes w_{\ell} .
\end{aligned}
$$

Then since $v=\sum_{i=1}^{k} u_{i} \otimes w_{i}$, and since $\mathcal{B}$ is a basis for $V$, we deduce that $\left(A_{0}^{T} B_{0}\right)_{j \ell}=0$ if at least one of $j>k, \ell>k, j \neq \ell$, and $\left(A_{0}^{T} B_{0}\right)_{j j}=1$ for $1 \leq j \leq k$. In particular this implies that each $x_{i}^{\prime} \in U_{0}$ and each $y_{i}^{\prime} \in W_{0}$. Moreover by part (i) the $x_{i}^{\prime}$ are linearly independent, and also the $y_{i}^{\prime}$ are linearly independent. Thus part (ii) follows. We have also proved part (iii).

Finally if $g=\left(A_{1}, B_{1}\right) \in X_{v}$ (modulo $\left.Z_{0}\right)$, with $A_{1}=\left(a_{i j}\right) \in G L(U)$ and $B_{1}=\left(b_{i j}\right) \in$ $G L(W)$, then we have $v=\sum_{i=1}^{k}\left(u_{i} A_{1}\right) \otimes\left(w_{i} B_{1}\right)$. By part (ii), for each $i \leq k, u_{i} A_{1} \in U_{0}$ and $w_{i} B_{1} \in W_{0}$, so $g \in X_{U_{0} \otimes W_{0}}$. Moreover, if $A=\left.A_{1}\right|_{U_{0}}, B=\left.B_{1}\right|_{W_{0}}$, written as matrices with respect to the bases $\left\{x_{1}, \ldots, x_{k}\right\}$ and $\left\{y_{1}, \ldots, y_{k}\right\}$ respectively, then by part (iii), $B=A^{-T}$ so $g$ is one of the elements described in part (iv). For each $A \in G L(k, q)$, there exists an element $\left(A_{1}, B_{1}\right) \in X$ with $A=\left.A_{1}\right|_{U_{0}}, A^{-T}=\left.B_{1}\right|_{W_{0}}$, and the fact that this element fixes $v$ follows from the displayed computation above. Finally $\hat{X}_{v}$ contains a conjugate of $\sigma$ which induces on $U_{0} \otimes W_{0}$ the natural field automorphism with respect to the basis formed by the $x_{i} \otimes y_{j}$.

By Lemma 4.3(ii), the subspaces $U_{0}$ and $W_{0}$ are determined uniquely by $v$, and we denote them by $U_{0}(v)$ and $W_{0}(v)$ respectively.

4.2. Proof of Theorem 4.1. Suppose $H \leq \hat{X}$ preserves a non-trivial tensor decomposition $V=U \otimes W$ of $V=\mathbb{F}_{q}^{n}$, and that $\hat{X}, X, Z_{0}, q=p^{f}, a, b$ are as in Subsection 4.1. Suppose also that $H$ is $p$-exceptional on $V$. By Lemma 2.2 we may assume that $H$ contains $Z:=$ $Z(G L(V))=\left(Z_{U} \times Z_{W}\right) / Z_{0}$, where $Z_{U}=Z(G L(U)), Z_{W}=Z(G L(W))$.

For Theorem 4.1 we will have $H \leq X$, but our first lemma is more general and will be used also for the proof of Theorem 4.2. The natural projection maps $\phi_{U}: \hat{X} \rightarrow P \Gamma L(U)$ and $\phi_{W}$ : $\hat{X} \rightarrow P \Gamma L(W)$ have kernels $K_{U}=Z_{U} \circ G L(W) \cong G L(W)$ and $K_{W}=G L(U) \circ Z_{W} \cong G L(U)$ respectively. Also for subspaces $U_{0} \leq U, W_{0} \leq W$, we have maps $\phi_{U_{0}}: \hat{X}_{U_{0} \otimes W} \rightarrow P \Gamma L\left(U_{0}\right)$ and $\phi_{W_{0}}: \hat{X}_{U \otimes W_{0}} \rightarrow P \Gamma L\left(W_{0}\right)$ with kernels $K_{U_{0}}, K_{W_{0}}$ respectively. If an element $x \in \hat{X}$ or subgroup $L \leq \hat{X}$ lies in $\hat{X}_{U_{0} \otimes W}$ then we write $x^{U_{0}}=\phi_{U_{0}}(x), L^{U_{0}}=\phi_{U_{0}}(L)$ for the corresponding element or subgroup of $P \Gamma L\left(U_{0}\right)$; also by the fixed point subspace of $L^{U}$ we mean the largest $L^{U}$-invariant subspace $U_{0}$ of $U$ such that $L^{U_{0}}=1$. Similarly for subspaces of $W$. A subgroup $L \leq \hat{X}_{U_{0} \otimes W_{0}}$ is said to act diagonally on $U_{0} \otimes W_{0}$ if $L \cap K_{U_{0}}$ and $L \cap K_{W_{0}}$ both induce only scalar transformations on $U_{0} \otimes W_{0}$.

Lemma 4.4. Suppose that $H \leq \hat{X}$ and $H$ is p-exceptional. Let $U_{0}, W_{0}$ be 2-dimensional subspaces of $U, W$ respectively, and let $P$ be a Sylow p-subgroup of $H_{U_{0} \otimes W_{0}}$. Then $P$ is a Sylow p-subgroup of $H$ (and so $P \neq 1$ ), $P$ acts diagonally on $U_{0} \otimes W_{0}$, and moreover $P \cap K_{U_{0}}=P \cap K_{W_{0}}=1$, and $P \cap X$ is elementary abelian of order at most $q$.

Proof Choose a weight 2 vector $v \in V$ such that $U_{0}(v)=U_{0}$ and $W_{0}(v)=W_{0}$. Then by Lemma 4.3, $H_{v} \leq H_{U_{0} \otimes W_{0}}$, and by our assumption $\left|H: H_{v}\right|$ is coprime to $p$. Thus $P$ is a Sylow $p$-subgroup of $H$, and in particular $P \neq 1$. Also, $P$ is conjugate in $H_{U_{0} \otimes W_{0}}$ to a Sylow $p$-subgroup $P^{\prime}$ of $H_{v}$. Since $P^{\prime}$ induces a diagonal action on $U_{0} \otimes W_{0}$ by Lemma 4.3(iv), it follows that also $P$ induces a diagonal action on $U_{0} \otimes W_{0}$.

Let $Q=P \cap K_{U_{0}}$ and assume that $Q \neq 1$. Since $P$ acts diagonally on $U_{0} \otimes W_{0}$, it follows that $Q^{W_{0}}=1$ (since $Q$ is a $p$-group), and we deduce that $Q=P \cap K_{W_{0}}$. Further, since $Q \neq 1$, we may assume without loss of generality that $Q^{U} \neq 1$. We produce an element $g \in Q$ and a 2-dimensional $\left\langle g^{U}\right\rangle$-invariant subspace $U_{0}^{\prime}$ such that $g^{U_{0}^{\prime}} \neq 1$ as follows: the fixed point subspace $U_{1}$ of the nontrivial $p$-group $Q^{U}$ contains $U_{0}$ and $U_{1} \neq U$, and there is a 1-dimensional subspace $U_{2} / U_{1}$ of $U / U_{1}$ left invariant by $Q^{U}$ in its induced quotient action on $U / U_{1}$. Since $Q^{U_{1}}=1$ and $Q^{U_{2}}$ is a nontrivial $p$-group, there exists $u \in U_{2} \backslash U_{1}$ and $g \in Q$ 
such that $\left\langle u^{g}\right\rangle=\langle u+x\rangle$ for some non-zero $x \in U_{1}$. Let $U_{0}^{\prime}=\langle u, x\rangle$, and note that $x^{g}=x$ since $x \in U_{1}$. Thus $U_{0}^{\prime}$ is invariant under $\left\langle g^{U}\right\rangle$ and has dimension 2 , and $g^{U_{0}^{\prime}} \neq 1$. Moreover since $g \in Q \subset P$, it follows that $g \in H_{U_{0}^{\prime} \otimes W_{0}}$ and that $g$ does not act diagonally on $U_{0}^{\prime} \otimes W_{0}$ since $g^{W_{0}}=1$. This contradicts the diagonal action of a Sylow $p$-subgroup of $H_{U_{0}^{\prime} \otimes W_{0}}$ on $U_{0}^{\prime} \otimes W_{0}$. Thus $P \cap K_{U_{0}}=P \cap K_{W_{0}}=1$. In particular $P$ is isomorphic to a subgroup of $P \Gamma L\left(U_{0}\right)$ and hence $P \cap X$ is elementary abelian of order at most $q$.

Corollary 4.5. Assume that $H \leq X$, and let $1 \neq g \in H$ be a p-element. Then $g^{U}$ and $g^{W}$ are both regular unipotent of order $p$.

Proof We may assume that $1 \neq g \in P \leq H_{U_{0} \otimes W_{0}}$ with $U_{0}, W_{0}, P$ as in Lemma 4.4. Then $P$ is elementary abelian so $|g|=p$, and $g^{U_{0}}, g^{W_{0}}$ are both nontrivial. Also $g^{U}, g^{W}$ have non-zero fixed point subspaces. Suppose that $U_{1}$ is a 2-dimensional subspace of the fixed point subspace of $g^{U}$. Then $g$ is a nontrivial $p$-element in $H_{U_{1} \otimes W_{0}}$, contradicting the diagonal action of $p$-elements proved in Lemma 4.4. Thus the fixed point subspace of $g^{U}$ has dimension 1 , so $g^{U}$ is regular unipotent. Similarly $g^{W}$ is regular unipotent.

In the next lemma we use the following notation. For a vector space $V$ of dimension at least $k$, let $P_{k}(V)$ denote the set of $k$-dimensional subspaces of $V$. Also let $\Gamma L_{1}^{*}\left(q^{p}\right)=$ $G L_{1}\left(q^{p}\right) . p$, where the cyclic group of order $p$ on top is generated by a field automorphism (so that $\left.\Gamma L_{1}^{*}\left(q^{p}\right) \leq G L_{p}(q)\right)$.

Lemma 4.6. Assume that $H \leq X$ and let $k \leq \min \{\operatorname{dim}(U), \operatorname{dim}(W)\}$. Then $H$ acts transitively on $P_{k}(U) \times P_{k}(W)$ (in its natural product action). Moreover $p=\operatorname{dim}(U)=$ $\operatorname{dim}(W) \in\{2,3\}$, and $H \leq \Gamma L_{1}^{*}\left(q^{p}\right) \circ \Gamma L_{1}^{*}\left(q^{p}\right)$.

Proof By Corollary 4.5, each element of order $p$ in $H$ fixes unique $k$-dimensional subspaces of $U$ and of $W$, and this property is true also for each Sylow $p$-subgroup of $H$. For $i=1,2$, let $U_{i}, W_{i}$ be a $k$-dimensional subspace of $U, W$ respectively, and let $v_{i}=\sum_{j=1}^{k} x_{i j} \otimes y_{i j} \in$ $U_{i} \otimes W_{i}$ be a weight $k$-vector so that the $x_{i j}$ span $U_{i}$ and the $y_{i j}$ span $W_{i}$. Let $P_{i}$ be a Sylow $p$-subgroup of $H_{v_{i}}$. Since $H$ is $p$-exceptional, $P_{i}$ is a Sylow $p$-subgroup of $H$, and by Lemma 4.3, $H_{v_{i}} \leq H_{U_{i} \otimes W_{i}}$. Thus there is an element $x \in H$ such that $P_{1}^{x}=P_{2}$, and hence $P_{2}$ fixes the $k$-subspaces $U_{1}^{x}, U_{2}$ of $U$ and $W_{1}^{x}, W_{2}$ of $W$. By uniqueness, we have $U_{1}^{x}=U_{2}$ and $W_{1}^{x}=W_{2}$. This proves the first assertion.

By [31, Lemma 4.1], for the group $\phi_{U}(H)$ to be transitive on $k$-subspaces, one of the following holds: (i) $\phi_{U}(H) \geq P S L(U)$, or (ii) $k \in\{1, \operatorname{dim}(U)-1\}$ and $\phi_{U}(H) \nsupseteq P S L(U)$, or (iii) $\operatorname{dim}(U)=5, p=2$ and $\left|\phi_{U}(H)\right|=31.5$. Since $p$ divides $\left|\phi_{U}(H)\right|$, case (iii) does not arise, and by Corollary $4.5, \operatorname{dim}(U)=2$ in case (i). The same comments apply to $\phi_{W}(H)$. Since we may always take $k=2$ in the previous paragraph, it follows that each of $a:=\operatorname{dim}(U)$ and $b:=\operatorname{dim}(W)$ is at most 3 . Then by the classification of transitive linear groups (see [30, Appendix] $)$, and noting that $p$ divides $\left|\phi_{U}(H)\right|$, either $\phi_{U}(H) \leq \Gamma L_{1}\left(q^{a}\right) / Z_{U}$, or $a=2$ and $\phi_{U}(H) \geq P S L(U)$, or $\mathrm{A}_{5} \leq \phi_{U}(H) \leq P \Gamma L_{2}(9)$. We have the same three possibilities for $\phi_{W}(H)$.

Suppose first that $\phi_{U}(H) \geq P S L(U)$ with $a=2$. Then since $P$ acts diagonally by Lemma 4.4, it follows that $b=2$ and $H$ has a single composition factor $P S L_{2}(q)$. However in this case $H$ is not transitive on $P_{1}(U) \times P_{1}(W)$. A similar argument rules out the third possibility. Thus $\phi_{U}(H) \leq \Gamma L_{1}\left(q^{a}\right) / Z_{U}$, and similarly $\phi_{W}(H) \leq \Gamma L_{1}\left(q^{b}\right) / Z_{W}$. Since $p$ divides the order of each of these groups we must have $p=a=b \in\{2,3\}$.

Next we show that the case $p=3$ does not yield a $p$-exceptional group. The proof and two further proofs use the following simple fact.

Remark 4.7. Suppose that $a=\operatorname{dim}(U)=\operatorname{dim}(W)$. Let $\left\{u_{1}, u_{2}, \ldots, u_{a}\right\}$ be a basis for $U$. By Lemma 4.3, each weight $a$ vector $v$ in $U \otimes W$ has a unique representation of the form $v=\sum_{i=1}^{a} u_{i} \otimes w_{i}$ where the $w_{i}$ form a basis for $W$. Thus the number of weight $a$ vectors is $\left|G L_{a}(q)\right|$.

Lemma 4.8. If $p=3$, and $H \leq \Gamma L_{1}^{*}\left(q^{p}\right) \circ \Gamma L_{1}^{*}\left(q^{p}\right)$, then $H$ is not p-exceptional. 
Proof Suppose that $H$ is as stated and that $H$ is $p$-exceptional. By Lemma 2.2, we may assume that $Z<H$, and by Lemma 4.4 it follows that a Sylow 3-subgroup $P$ of $H$ has order 3 and acts diagonally on $U \otimes W$. Let $r:=q^{2}+q+1$. By Lemma 4.6, $r^{2}$ divides $|H|$. Also, since $\operatorname{gcd}(r, q-1)=1$, it follows that $H=Z \times\left(Z_{r}^{2} \cdot P\right)$. Now $P$ centralises $Z$ and $N_{H}(P)=Z P$ so that $H$ has exactly $r^{2}$ Sylow 3 -subgroups.

The group $P$ in its action on $U$ leaves invariant a unique 2-subspace $U_{2}$. The same is true for the $P$-action on $W$. Let $\left\{u_{1}, u_{2}, u_{3}\right\}$ be a $P$-orbit forming a basis for $U$. By Lemma 4.3, each weight 3 vector $v$ in $U \otimes W$ has a unique representation of the form $v=\sum_{i=1}^{3} u_{i} \otimes w_{i}$ where the $w_{i}$ form a basis for $W$, and it is straightforward to show that $v$ is fixed by $P$ if and only if the $w_{i}$ form a $P$-orbit in $W$; now each such $P$-orbit yields three weight 3 vectors fixed by $P$. Hence the number of weight 3 vectors fixed by $P$ is exactly $q^{3}-q^{2}$. By Remark 4.7 , the number of weight 3 vectors is $\left|G L_{3}(q)\right|$, and since each weight 3 vector is fixed by some Sylow 3-subgroup of $H$, it follows that $H$ has at least $\left|G L_{3}(q)\right| /\left(q^{3}-q^{2}\right)$ Sylow 3 -subgroups. Since $r^{2}<\left|G L_{3}(q)\right| /\left(q^{3}-q^{2}\right)$, this is a contradiction.

Finally we show that the case $p=2$ does lead to (reducible) $p$-exceptional examples. This result completes the proof of Theorem 4.1 .

Lemma 4.9. If $p=2$, and $H \leq \Gamma L_{1}^{*}\left(q^{2}\right) \circ \Gamma L_{1}^{*}\left(q^{2}\right)$, then $Z H$ is 2-exceptional if and only if $Z H=\left(G L_{1}\left(q^{2}\right) \circ G L_{1}\left(q^{2}\right)\right) .2$, an index 2 subgroup of $\Gamma L_{1}^{*}\left(q^{2}\right) \circ \Gamma L_{1}^{*}\left(q^{2}\right)$, with the Sylow 2 -subgroups acting diagonally. This group $Z H$ is reducible and 2-exceptional, with two orbits of length $q^{2}-1$ and $q-1$ orbits of length $\left(q^{2}-1\right)(q+1)$ on non-zero vectors.

Proof Suppose that $H$ is as stated and that $H$ is 2-exceptional. By Lemma 2.2, we may assume also that $Z<H$. Arguing as in the proof of Lemma 4.8, a Sylow 2-subgroup $P$ has order 2 and acts diagonally on $U \otimes W, N_{H}(P)=Z P$, and $H=Z \times\left(Z_{q+1}^{2} . P\right)=$ $\left(G L_{1}\left(q^{2}\right) \circ G L_{1}\left(q^{2}\right)\right) .2$.

Conversely suppose that $H=\left(G L_{1}\left(q^{2}\right) \circ G L_{1}\left(q^{2}\right)\right) .2$ with each Sylow 2-subgroup $P$ acting diagonally. Identify $U$ and $W$ with $\mathbb{F}_{q^{2}}$ and let $H_{1}$ be the index 2 subgroup of $H$ so that $H_{1}$ acts by field multiplication on both factors. Then $H=H_{1}\langle\tau\rangle$ where $\tau$ acts on both factors as the field automorphism of order 2. Let $\omega \in \mathbb{F}_{q^{2}}$ have order $q+1$ and note that $\omega^{2}=1+\lambda \omega$ where $\lambda=\omega+\omega^{q} \in \mathbb{F}_{q}$.

Let $v=1 \otimes 1+\omega \otimes \omega$ and $w=1 \otimes \omega+\omega \otimes 1$. Let $X=G L_{1}\left(q^{2}\right) \circ I$ and note that $v^{X}=\left\{\xi \otimes 1+\xi \omega \otimes \omega \mid \xi \in \mathbb{F}_{q^{2}}^{*}\right\}$ is a set of size $q^{2}-1$ and forms the set of nonzero vectors of an $\mathbb{F}_{q}$-subspace $U_{1}$ of $V$. Similarly, $w^{X}=\left\{\xi \otimes \omega+\xi \omega \otimes 1 \mid \xi \in \mathbb{F}_{q^{2}}^{*}\right\}$ is also the set of $q^{2}-1$ nonzero vectors of an $\mathbb{F}_{q}$-subspace $U_{2}$. Moreover, $U_{1} \cap U_{2}=\{0\}$. Note that an element of $X$ induces multiplication by the same element of $\mathbb{F}_{q^{2}}^{*}$ on each of $U_{1}$ and $U_{2}$.

Let $g \in H$ be the element that multiplies by $\omega$ in the both the first and second factors. Then

$$
\begin{aligned}
w^{g} & =\omega \otimes \omega^{2}+\omega^{2} \otimes \omega \\
& =\omega \otimes(1+\lambda \omega)+(1+\lambda \omega) \otimes \omega \\
& =\omega \otimes 1+(\lambda \omega+1+\lambda \omega) \otimes \omega=w
\end{aligned}
$$

Thus $\left|w^{H_{1}}\right| \leq q^{2}-1$ but since $w^{X} \subseteq w^{H_{1}}$ it follows that $w^{H_{1}}$ has size $q^{2}-1$. Also

$$
\begin{aligned}
(\xi \otimes \omega+\xi \omega \otimes 1)^{\tau} & =\xi^{q} \otimes \omega^{q}+\xi^{q} \omega^{q} \otimes 1 \\
& =\xi^{q} \otimes(\lambda+\omega)+\xi^{q}(\lambda+\omega) \otimes 1 \\
& =\xi^{q} \otimes \omega+\left(\xi^{q} \lambda+\xi^{q} \lambda+\xi^{q} \omega\right) \otimes 1 \\
& =\xi^{q} \otimes \omega+\xi^{q} \omega \otimes 1 \in U_{2}
\end{aligned}
$$

Hence $U_{2}$ is $H$-invariant.

Similar calculations, taking $g$ to be the element that multiplies the first factor by $\omega$ and the second by $\omega^{q}$, show that $U_{1}$ is also $H$-invariant.

Let $C$ be the subgroup of $G L_{1}\left(q^{2}\right) \times G L_{1}\left(q^{2}\right)$ acting on $U_{1} \oplus U_{2}$ given by

$$
C:=\left\{(\alpha, \beta) \mid \alpha, \beta \in \mathbb{F}_{q^{2}}^{*},\left(\alpha \beta^{-1}\right)^{q+1}=1\right\}
$$


Note that $|C|=\left(q^{2}-1\right)(q+1)=\left|H_{1}\right|$. We have already seen that elements of $X$ are elements of $C$. Now consider elements $y=(1, \xi)$ of $Y=I \circ G L_{1}\left(q^{2}\right) \leq H_{1}$ and let $a, b \in \mathbb{F}_{q}$ such that $\xi=a+b \omega$. Then

$$
\begin{aligned}
(1 \otimes 1+\omega \otimes \omega)^{y} & =1 \otimes(a+b \omega)+\omega \otimes(a+b \omega) \omega \\
& =a \otimes 1+b \otimes \omega+a \omega \otimes \omega+b \omega \otimes(1+\lambda \omega) \\
& =(a+b \omega) \otimes 1+(b+a \omega+b \lambda \omega) \otimes \omega \\
& =\xi \otimes 1+\xi \omega \otimes \omega
\end{aligned}
$$

and so $y$ induces multiplication by $\xi$ on $U_{1}$. Similarly, $(1 \otimes \omega+\omega \otimes 1)^{y}=\xi^{q} \otimes \omega+\xi^{q} \omega \otimes 1$ and so $y$ induces multiplication by $\xi^{q}$ on $U_{2}$. Thus the elements of $Y$ are also elements of $C$. Since each element of $H_{1}$ is the product of an element of $X$ and an element of $Y$, comparing orders yields $C=H_{1}$. We have already seen that $H_{1}$ has two orbits of length $q^{2}-1\left(U_{1}^{\sharp}\right.$ and $U_{2}^{\sharp}$ ), and using the fact that $H_{1}=C$ we see that it also has $q-1$ orbits of length $\left(q^{2}-1\right)(q+1)$, namely

$$
\Delta_{\lambda}:=\left\{(u, v) \mid u, v \in \mathbb{F}_{q^{2}}^{*},\left(u v^{-1}\right)^{q+1}=\lambda\right\}
$$

for $\lambda \in \mathbb{F}_{q}^{*}$, on $V^{\sharp}$. Also consider $s_{1}, s_{2} \in G L(V)$, where $s_{1}$ sends $(u, v) \in V$ to $\left(u^{q}, v\right)$ and $s_{2}$ sends $(u, v)$ to $\left(u, v^{q}\right)$. Then $\tilde{C}:=\left\langle C, s_{1}, s_{2}\right\rangle \cong C: 2^{2}$ has the same orbits as $C$ does on $V$. Moreover, $H=C:\left\langle s_{1} s_{2}\right\rangle$.

4.3. Proof of Theorem 4.2. Suppose now that $H \leq \Gamma L_{n}\left(q^{p}\right)$ acting on $V=\mathbb{F}_{q^{p}}^{n}$, is $p$ exceptional, that $p=|H: H \cap G L(V)|$, and that $|H \cap G L(V)|$ is coprime to $p$. Suppose moreover that $H \leq X:=\operatorname{Stab}_{\Gamma L(V)}(U \otimes W)$, where $a=\operatorname{dim}(U) \geq 2, b=\operatorname{dim}(W) \geq 2$ with $a \leq b$, and set $r=q^{p}$. By Lemma 2.2, we may assume that $H$ contains $Z=Z(G L(V))$. Theorem 4.2 follows from the following lemma.

Lemma 4.10. $p=a=2$.

Proof Choose 2-dimensional subspaces $U_{0}, W_{0}$ of $U, W$ respectively, set $V_{0}:=U_{0} \otimes W_{0}$, and consider the subgroup $L$ of $\operatorname{Stab}_{\Gamma L\left(V_{0}\right)}\left(U_{0} \otimes W_{0}\right)$ induced by $H_{U_{0} \otimes W_{0}}$. Let $\Delta$ denote the set of weight 2 vectors of $U_{0} \otimes W_{0}$ (considered as vectors of $V$ ), and let $v \in \Delta$. By Lemma 4.3, $H_{v} \leq H_{U_{0} \otimes W_{0}}$, and since $H$ is $p$-exceptional $\left|H: H_{v}\right|$ is coprime to $p$. It follows from Lemma 4.4 that $p$ divides $|L|$ and a Sylow $p$-subgroup $P$ of $L$ acts diagonally on $U_{0} \otimes W_{0}$. We may assume that $P$ acts as a group of field automorphisms of order $p$. Then the set of fixed points of $P$ in $U_{0} \otimes W_{0}$ forms an $\mathbb{F}_{q}$-space $U_{0}^{\prime} \otimes W_{0}^{\prime}=\mathbb{F}_{q}^{2} \otimes \mathbb{F}_{q}^{2}$. In particular we may choose a basis $u_{1}, u_{2}$ for $U_{0}$ from $U_{0}^{\prime}$.

By Remark 4.7, $|\Delta|=\left|G L_{2}(r)\right|$. Also each $v^{\prime} \in \Delta$ has a unique expression as $v^{\prime}=$ $u_{1} \otimes w_{1}+u_{2} \otimes w_{2}$ where $w_{1}, w_{2}$ span $W_{0}$, and it is straightforward to prove that $P$ fixes $v^{\prime}$ if and only if $P$ fixes $w_{1}$ and $w_{2}$, that is to say, if and only if $v^{\prime} \in U_{0}^{\prime} \otimes W_{0}^{\prime}$. Thus $P$ fixes exactly $\left|G L_{2}(q)\right|$ vectors in $\Delta$. Since each $v^{\prime} \in \Delta$ is fixed by at least one Sylow $p$-subgroup of $L$ (by $p$-exceptionality), it follows that the number $\left|L: N_{L}(P)\right|$ of Sylow $p$-subgroups of $L$ is at least

$$
y:=\frac{\left|G L_{2}(r)\right|}{\left|G L_{2}(q)\right|}=\frac{\left(r^{2}-1\right)\left(r^{2}-r\right)}{\left(q^{2}-1\right)\left(q^{2}-q\right)} .
$$

Let $Z_{0}=Z\left(G L\left(V_{0}\right)\right)$, and note that $Z_{0}$ is contained in $L_{0}:=L \cap G L\left(V_{0}\right)$ since $H$ contains $Z$. Now $N_{L}(P) \cap Z_{0} \cong Z_{q-1}$, and $N_{L}(P)$ contains $L \cap\left(G L\left(U_{0}^{\prime}\right) \circ G L\left(W_{0}^{\prime}\right)\right)$.

Recall the definitions of the maps $\phi_{U_{0}}, \phi_{W_{0}}$ at the beginning of Section 4.2. From the classification of subgroups of $P G L_{2}(r)$ (see [10, Chapter XII]), it follows that the $p^{\prime}$-group $L_{0}$ is such that each of $\phi_{U_{0}}\left(L_{0}\right), \phi_{W_{0}}\left(L_{0}\right)$ is either a subgroup of $D_{2(r \pm 1)}$ or equals one of $\mathrm{A}_{4}, \mathrm{~S}_{4}, \mathrm{~A}_{5}$. In the latter three cases, $p$ would be odd, and such subgroups would lie in a subfield subgroup $P G L_{2}(q)$, and be centralised by $P$. Thus if the latter cases held for at least one of $\phi_{U_{0}}\left(L_{0}\right), \phi_{W_{0}}\left(L_{0}\right)$, then we would have $\left|L: N_{L}(P)\right| \leq\left|Z: N_{Z}(P)\right| \cdot 2(r+1)=$ $2\left(r^{2}-1\right) /(q-1)$, which is strictly less than $y$, giving a contradiction. Thus $L_{0} / Z_{0} \leq$ $D_{2(r+\varepsilon)} \times D_{2\left(r+\varepsilon^{\prime}\right)}$, for some $\varepsilon, \varepsilon^{\prime} \in\{1,-1\}$. 
Suppose first that $p$ is odd. Then $N_{L_{0}}(P) / N_{Z_{0}}(P) \leq D_{2(q+\varepsilon)} \times D_{2\left(q+\varepsilon^{\prime}\right)}$, and we find

$$
\left|L: N_{L}(P)\right| \leq \frac{r-1}{q-1} \cdot \frac{r+\varepsilon}{q+\varepsilon} \cdot \frac{r+\varepsilon^{\prime}}{q+\varepsilon^{\prime}} \leq\left(\frac{r-1}{q-1}\right)^{3}
$$

which is less than $y$, contradiction. Hence $p=2$.

Suppose now that $a=\min \{a, b\} \geq 3$. We may repeat the above analysis with 3dimensional subspaces $U_{0}, W_{0}$ and $\Delta$ the set of weight 3 vectors in $V_{0}=U_{0} \otimes W_{0}$ : the cardinality $|\Delta|$ is $\left|G L_{3}(r)\right|, P$ fixes $\left|G L_{3}(q)\right|$ vectors in $\Delta$, and the number of Sylow 2subgroups of $L$ is at least

$$
y^{\prime}:=\frac{\left|G L_{3}(r)\right|}{\left|G L_{3}(q)\right|}=\frac{\left(r^{3}-1\right)\left(r^{3}-r\right)\left(r^{3}-r^{2}\right)}{\left(q^{3}-1\right)\left(q^{3}-q\right)\left(q^{3}-q^{2}\right)} .
$$

Again $L_{0}:=L \cap G L\left(V_{0}\right)$ is such that each of $\phi_{U_{0}}\left(L_{0}\right)$ and $\phi_{W_{0}}\left(L_{0}\right)$ is an odd order subgroup of $G L_{3}(r)$, and hence is completely reducible. Thus each of these subgroups is a subgroup of one of $Z_{r^{2}+r+1} \cdot 3,\left(Z_{r^{2}-1} \times Z_{r-1}\right) / Z_{0}$ or $Z_{r-1}^{3} / Z_{0}$, and it follows that

$$
\left|L: N_{L}(P)\right| \leq \frac{r-1}{q-1} \cdot\left(\max \left\{\frac{r^{2}+r+1}{q^{2}+q+1},(r+1) \frac{r-1}{q-1},\left(\frac{r-1}{q-1}\right)^{2}\right\}\right)^{2}
$$

which equals $(q+1)^{5}$ (recall that $r=q^{2}$ here). However this quantity is less than $y^{\prime}$ and we have a contradiction. Thus $a=2$.

\section{Tensor products II: $\mathcal{C}_{7}$ CASE}

In this section we classify $p$-exceptional groups which preserve tensor-induced decompositions. By this we mean the following. Let $V_{1}$ be a vector space over $\mathbb{F}_{q}$, and let $V=V_{1}^{\otimes t}=V_{1} \otimes V_{2} \otimes \cdots \otimes V_{t}$, a tensor product of $t$ spaces isomorphic to $V_{1}$. The group $\left(G L\left(V_{1}\right) \circ \cdots \circ G L\left(V_{t}\right)\right) . \mathrm{S}_{t}$ acts on $V$, where all centres are identified in the central product and the group $\mathrm{S}_{t}$ permutes the tensor factors. If $G$ is a subgroup of this group we say that $G$ preserves the tensor-induced decomposition $V=V_{1}^{\otimes t}$.

Theorem 5.1. Assume $G<G L(V)$ is a (not necessarily irreducible) p-exceptional group which preserves a tensor-induced decomposition

$$
\mathbb{F}_{q}^{n}=V=\left(V_{1}\right)^{\otimes t}=V_{1} \otimes V_{2} \otimes \ldots \otimes V_{t},
$$

where $\operatorname{dim}_{\mathbb{F}_{q}} V_{i}=m \geq 2$ and $t \geq 2$. Then $p=2$, and one of the following holds:

(i) $t=4$ and $m=q=2$;

(ii) $t=3$, and $m=2,3$. Moreover, if $m=3$ then $q=2$, and if $m=2$ then $q \leq 4$;

(iii) $t=2$, and $m=2,3$. Moreover, if $m=3$ then $q \leq 8$.

We shall also need the following result for the semilinear case.

Theorem 5.2. Let $G \leq \Gamma L(V)$, and assume that $G_{0} \triangleleft G=\left\langle G_{0}, \sigma\right\rangle$, where

: (i) $G_{0}$ is an absolutely irreducible $p^{\prime}$-subgroup of $G L(V)$ which preserves a tensorinduced decomposition

$$
V=\left(V_{1}\right)^{\otimes t}=V_{1} \otimes V_{2} \otimes \ldots \otimes V_{t},
$$

with $V=\mathbb{F}_{q}^{n}, \operatorname{dim}_{\mathbb{F}_{q}} V_{i}=m \geq 2, t \geq 2$, and

: (ii) $q=p^{p f}$, and $\sigma$ induces the field automorphism $x \mapsto x^{p^{f}}$ of $V$ modulo $G L(V)$.

Then $G$ is not p-exceptional.

The following result classifies the $p$-exceptional examples occurring in the cases left over by Theorem 5.1 .

Proposition 5.3. Let $G<G L(V)$ be p-exceptional as in the hypothesis of Theorem 5.1, and suppose $t, m, q$ are as in one of conclusions (i)-(iii) of the theorem. Suppose also that $G$ is irreducible on $V$. Then one of the following holds: 
(a) $m=3, t=2, q=2$ : there are two irreducible 2-exceptional groups $G$, of the form $7^{2} . \mathrm{S}_{3}$ (orbit lengths $1,21,49^{7}, 147$ ) and $(7.3)^{2} .2$ (orbit lengths $1,21,49,147^{3}$ ); both are imprimitive.

(b) $m=2, t=2$ : any irreducible 2-exceptional group $G$ in this case is conjugate to a subgroup of $G L_{2}\left(q^{2}\right)$, hence is given by Lemma 2.8 .

The proofs of these results are presented in the following three subsections.

5.1. Proof of Theorem 5.1. Throughout this section we assume that $G \leq G L(V)$ is a (not necessarily irreducible) $p$-exceptional group which preserves a tensor-induced decomposition

$$
V=\left(V_{1}\right)^{\otimes t}=V_{1} \otimes V_{2} \otimes \ldots \otimes V_{t},
$$

where $V=\mathbb{F}_{q}^{n}, \operatorname{dim}_{\mathbb{F}_{q}} V_{i}=m \geq 2, t \geq 2$, and $(m, t, p) \neq(2,2,2)$. Let $B:=G \cap\left(G L\left(V_{1}\right) \circ\right.$ $\left.\ldots \circ G L\left(V_{t}\right)\right)$ be the base group and let $H=G / B \leq \mathrm{S}_{t}$ be the permutation group induced by the action of $G$ on the $t$ tensor factors $V_{i}$.

5.1.1. First reduction. We begin with some elementary observations. Recall that a rational element of a finite group is an element which is conjugate to all of its powers which have the same order.

Lemma 5.4. Under the above hypothesis, the following statements hold.

(i) $B$ is a $p^{\prime}$-group.

(ii) $H$ is a transitive subgroup of $\mathrm{S}_{t}$ of order divisible by $p$. In particular $t \geq p$.

(iii) Let $1 \neq h \in G$ be any p-element and let $Q \leq G$ be any $p$-subgroup containing $h$. Then

$$
\left|G: N_{G}(Q)\right|>\left|V / C_{V}(h)\right| \text {. }
$$

(iv) $G \backslash B$ contains an element $g$ of order $p$, and for such an element,

$$
\frac{|G|}{p \cdot\left|C_{B}(g)\right|}>\left|V / C_{V}(g)\right| \text {. }
$$

If in addition the element $g B$ is rational in $H=G / B$, then

$$
\frac{|G|}{p(p-1) \cdot\left|C_{B}(g)\right|}>\mid V / C_{V}(g \mid .
$$

Proof If $p$ divides $|B|$ then $B$ is $p$-exceptional by Lemma 2.1, which contradicts Theorem 4.1 (since we are assuming that $(m, t, p) \neq(2,2,2)$ ). Part (i) follows. Likewise, if $H$ is intransitive, then $G$ preserves a nontrivial tensor decomposition of $V$ and we get a contradiction by the same result; hence (ii) holds. Next, the $p$-exceptionality of $G$ implies that any nonzero element $v \in V$ is fixed by a Sylow $p$-subgroup of $G$ and so by a conjugate of $Q$ as well. Hence,

$$
|V|-1=\left|V^{\sharp}\right| \leq\left|G: N_{G}(Q)\right| \cdot\left|C_{V}(Q)\right| \leq\left|G: N_{G}(Q)\right| \cdot\left|C_{V}(h)\right|,
$$

and (iii) follows. Since $p$ divides $|G|$ and $B$ is a $p^{\prime}$-group, we can find $g \in G \backslash B$ of order $p$. Now we choose $h:=g$ and $Q:=\langle g\rangle$ in (iii). Observe that $C_{G}(g)$ contains $g$ and the $p^{\prime}$-subgroup $C_{B}(g)$, whence the first inequality in (iv) follows. Finally, since $B$ is a $p^{\prime}$-group, the rationality of $g B$ in $G / B$ implies $g$ is rational in $G$ (see e.g. [42, Lemma 4.11]), in which case we have $\left|N_{G}(Q)\right|=(p-1)\left|C_{G}(g)\right|$. Hence the second inequality in (iv) follows.

We fix the element $g$ in Lemma $5.4\left(\right.$ iv) and bound $\kappa:=\left(\operatorname{dim} C_{V}(g)\right) /(\operatorname{dim} V)$. Replacing $G$ by some conjugate subgroup, we may assume that $g$ permutes $V_{1}, \ldots, V_{p}$ cyclically:

$$
g: V_{1} \mapsto V_{2} \mapsto V_{3} \mapsto \ldots \mapsto V_{p} \mapsto V_{1}
$$

Choose a basis $\left(e_{j}^{1} \mid 1 \leq j \leq m\right)$ of $V_{1}$ and let $e_{j}^{i}:=\left(e_{j}^{1}\right) g^{i-1}$ for $1 \leq i \leq p$. Since $|g|=p$, we see that $\left(e_{j}^{p}\right) g=e_{j}^{1}$ and $\left(e_{j}^{i} \mid 1 \leq j \leq m\right)$ is a basis of $V_{i}$ for $1 \leq i \leq p$. Clearly,

$$
\left(e_{j_{1}}^{1} \otimes e_{j_{2}}^{2} \otimes \ldots \otimes e_{j_{p}}^{p} \mid 1 \leq j_{1}, \ldots, j_{p} \leq m\right)
$$

is a basis of $U:=V_{1} \otimes \ldots \otimes V_{p}$. Let $J_{k}$ denote the Jordan block of size $k$ with eigenvalue 1. Then $g \downarrow U$ permutes the above basis vectors of $U$ in $\left(m^{p}-m\right) / p$ cycles of length 
$p$, hence has Jordan canonical form $\left(J_{1}^{a}, J_{p}^{b}\right)$, where $a:=m, b:=\left(m^{p}-m\right) / p$. Also let $W:=V_{p+1} \otimes \ldots \otimes V_{t}$ so that $V=U \otimes W$.

Define $\kappa:=\frac{\operatorname{dim} C_{V}(g)}{\operatorname{dim} V}$, and observe that $\left|V / C_{V}(g)\right|=|V|^{1-\kappa}$.

Lemma 5.5. We have

$$
\kappa=\frac{\operatorname{dim} C_{V}(g)}{\operatorname{dim} V} \leq \frac{1}{p}+\frac{1-\frac{1}{p}}{m^{p-1}} .
$$

Proof Consider any indecomposable direct summand $W^{\prime}$ of the $\langle g\rangle$-module $W$. Suppose $g$ acts on $W^{\prime}$ via Jordan block $J_{k}$. Then $g$ acts on $U \otimes W^{\prime}$ with Jordan canonical form $\left(J_{1}^{a}, J_{p}^{b}\right) \otimes J_{k}=\left(J_{k}^{a}, J_{p}^{b k}\right)$. It follows using the values of $a, b$ above, that

$$
\frac{\operatorname{dim} C_{U \otimes W^{\prime}}(g)}{\operatorname{dim}\left(U \otimes W^{\prime}\right)}=\frac{a+b k}{k(a+b p)} \leq \frac{a+b}{a+b p}=\frac{\operatorname{dim} C_{U}(g)}{\operatorname{dim} U}=\frac{1}{p}+\frac{1-\frac{1}{p}}{m^{p-1}} .
$$

Applying this observation to every indecomposable direct summand $W^{\prime}$ of the $\langle g\rangle$-module $W$, we get $\kappa \leq(a+b) /(a+b p)$, yielding the desired inequality.

Next we estimate $\left|B: C_{B}(g)\right|$.

Lemma 5.6. Let $X$ be a $p^{\prime}$-subgroup of $P G L_{m}(q)$ of largest possible order.

(i) Let $h \in G$ be an arbitrary element. Then

$$
\left|B: C_{B}(h)\right| \leq|X|^{t} \leq\left(\left|P G L_{m}(q)\right|_{p^{\prime}}\right)^{t} .
$$

(ii) If, in addition, $g$ acts trivially on $W$, then

$$
\left|B: C_{B}(g)\right| \leq|X|^{p-1} \leq\left(\left|P G L_{m}(q)\right|_{p^{\prime}}\right)^{p-1} .
$$

Proof Recall that for a tensor product space $\mathbb{F}_{q}^{k} \otimes \mathbb{F}_{q}^{l}$, if $A, C \in G L_{k}(q)$ and $D, E \in G L_{l}(q)$ are such that $A \otimes D=C \otimes E$, then $C=\alpha A$ and $E=\alpha^{-1} D$ for some $\alpha \in \mathbb{F}_{q}^{*}$. It follows that the map

$$
f: B \rightarrow P G L\left(V_{1}\right) \times \ldots \times P G L\left(V_{t}\right),
$$

defined by $f(x)=\left(\bar{A}_{1}, \ldots \bar{A}_{t}\right)$ if $x=A_{1} \otimes A_{2} \otimes \ldots \otimes A_{t} \in B, A_{i} \in G L\left(V_{i}\right)$, and $\bar{A}_{i}$ denotes the coset containing $A_{i}$ in $P G L\left(V_{i}\right)$, is a well-defined homomorphism. Observe that each fibre of $f$ is contained in exactly one $C_{B}(h)$-coset in $B$. Indeed, if $f(x)=f\left(x^{\prime}\right)$, then $x^{\prime}=\beta x$ for some $\beta \in \mathbb{F}_{q}^{*}$, and so $x^{\prime} x^{-1} \in C_{B}(h)$. Furthermore, since each $x \in B$ is a $p^{\prime}$-element by Lemma 5.4(i), the elements $\bar{A}_{i}$ are $p^{\prime}$-elements in $P G L\left(V_{i}\right)$. Composing $f$ with the projection $P G L\left(V_{1}\right) \times \ldots \times P G L\left(V_{t}\right) \rightarrow P G L\left(V_{i}\right)$, we therefore get a homomorphism $f_{i}: B \rightarrow$ $P G L\left(V_{i}\right)$ with $f_{i}(B)$ being a $p^{\prime}$-group. It follows that $\left|f_{i}(B)\right| \leq|X| \leq\left|P G L_{m}(q)\right|_{p^{\prime}}$. Now $|f(B)| \leq \prod_{i=1}^{t}\left|f_{i}(B)\right|$, whence (i) follows.

For (ii), notice that

$$
Y_{i}:=\left\{C \in G L\left(V_{i}\right) \mid C=A_{i} \text { for some } h=A_{1} \otimes \cdots \otimes A_{t} \in B\right\}
$$

is a $p^{\prime}$-subgroup of $G L\left(V_{i}\right)$. Given the action of $g$ on $V_{1}, \ldots, V_{p}$, we can identify $Y_{i}, 1 \leq i \leq p$, with $Y_{1}$. Consider the homomorphism

$$
f^{*}: Y:=Y_{1} \times \ldots \times Y_{t} \rightarrow Y_{1} \circ \cdots \circ Y_{t}
$$

given by $f^{*}\left(A_{1}, \ldots, A_{t}\right)=A_{1} \otimes \ldots \otimes A_{t}$, and note that $B \leq f^{*}(Y)$. Let

$$
\begin{aligned}
K & :=\left\{(\underbrace{A, \ldots, A}_{p}, D_{p+1}, \ldots, D_{t}) \mid A \in Y_{1}, D_{i} \in Y_{i}\right\}, \\
Z_{0} & :=\left\{\left(a_{1} I_{m}, \ldots, a_{t} I_{m}\right) \mid a_{i} \in \mathbb{F}_{q}^{*}\right\} .
\end{aligned}
$$

Since the element $g$ of order $p$ acts trivially on $W$ and permutes $V_{1}, \ldots, V_{p}$ cyclically, $f^{*}\left(K Z_{0}\right)$ centralizes $g$. Thus $f^{*}\left(K Z_{0}\right) \leq C_{f^{*}(Y)}(g)$ and

$$
\left|B: C_{B}(g)\right|=\left|g^{B}\right| \leq\left|g^{f^{*}(Y)}\right| \leq \frac{\left|f^{*}(Y)\right|}{\left|f^{*}\left(K Z_{0}\right)\right|}=\frac{|Y|}{\left|K Z_{0}\right|}=\left(\frac{\left|Y_{1}\right|}{q-1}\right)^{p-1} .
$$

It remains to observe that $\left|Y_{1}\right| \leq(q-1)|X|$. 
Lemma 5.7. Under the above assumptions, one of the following holds.

(i) $p=3$ and $(m, t)=(2,4)$, $(2,3)$.

(ii) $p=2$. Furthermore, either $t=2$ or $(m, t)=(4,3),(3,3),(2,6),(2,5),(2,4),(2,3)$.

Proof By Lemma 5.6, for $g$ the element defined before Lemma 5.5 we have

$$
\begin{aligned}
\left|G: C_{B}(g)\right| & \left.\leq|H| \cdot \mid B: C_{B}(g)\right] \leq(t !) \cdot\left(\left|P G L_{m}(q)\right|_{p^{\prime}}\right)^{t} \\
& <\left(\frac{t+1}{2} \cdot q^{m(m+1) / 2-1}\right)^{t}<|V|^{f(m, t, q)},
\end{aligned}
$$

where

$$
f(m, t, q)=t \cdot \frac{m(m+1) / 2+\log _{q} \frac{t+1}{2}-1}{m^{t}} .
$$

In particular, if $t \geq 5$, then $f(m, t, q)<0.6$. By Lemma 5.4(iv),

$$
\left|G: C_{B}(g)\right|>p\left|V / C_{V}(g)\right|>q^{\operatorname{dim} V-\operatorname{dim} C_{V}(g)}=|V|^{1-\kappa},
$$

and so $f(m, t, q)+\kappa>1$.

If $p \geq 5$, then $t \geq 5$ by Lemma 5.4 (ii) and so $f(m, t, q)<0.6$. Then by Lemma 5.5 , $\kappa \leq 1 / 4$, a contradiction.

Now assume that $p=3$, so $t \geq 3$ by Lemma 5.4 (ii). If $t \geq 5$, then $f(m, t, q)<0.47$. If $t=4$ and $m \geq 3$, then $f(m, t, q)<0.3$. If $t=3$ and $m \geq 4$, then $f(m, t, q)<0.46$. In all these cases $\kappa \leq 1 / 2$, and we arrive at a contradiction as above.

Consider the case $m=t=3$ (still with $p=3$ ). If $q \geq 9$, then $f(m, t, q)+\kappa<$ $0.5907+11 / 27<1$, again a contradiction. Assume $q=3$. Then $\left|C_{V}(g)\right| \leq 3^{11}$ by Lemma 5.5. On the other hand, by Lemma 5.6 we have

$$
\left|G: C_{B}(g)\right| \leq 2 \cdot\left(\left|P G L_{3}(3)\right|_{3^{\prime}}\right)^{3}<3^{16},
$$

contradicting Lemma 5.4(iv). The remaining cases are listed in (i).

Now we consider the case $p=2$. If $t \geq 7$, then $f(m, t, q)+\kappa<0.22+0.75<1$. If $m \geq 3$ and $t \geq 4$, then $f(m, t, q)+\kappa<0.32+2 / 3<1$. And if $m \geq 5$ and $t \geq 3$, then $f(m, t, q)+\kappa<0.36+0.6<1$. The remaining cases are listed in (ii).

5.1.2. The case $p=3$.

Proposition 5.8. The case $p=3$ is impossible.

Proof Observe that the subgroup $X$ in Lemma 5.6 has order $2(q+1)$ by Lemma 2.6(i), and $\kappa \leq \frac{1}{2}$ by Lemma 5.5. So $\left|V / C_{V}(g)\right| \geq|V|^{1-\kappa} \geq q^{m^{t} / 2}$. By Lemma 5.7 we need to consider two cases.

(1) Suppose $(m, t)=(2,4)$. In this case, Lemma 5.4(iv) and Lemma 5.6 imply

$$
q^{8} \leq\left|V / C_{V}(g)\right|<8 \cdot(2(q+1))^{4},
$$

whence $q=3$. Assume in addition that $g$ acts nontrivially on $V_{4}=\mathbb{F}_{3}^{2}$, i.e. $V_{4} \downarrow g=J_{2}$. Then

$$
V \downarrow g=\left(J_{1}^{2}, J_{3}^{2}\right) \otimes J_{2}=\left(J_{2}^{2}, J_{3}^{4}\right)
$$

and so Lemma 5.4(iv) implies

$$
q^{10} \leq\left|V / C_{V}(g)\right|<8 \cdot(2(q+1))^{4}
$$

again a contradiction.

Thus $g$ acts trivially on $V_{4}=W$. In this case, by Lemma 5.6 we have $q^{8}<\left|G: C_{G}(g)\right| \leq$ $8 \cdot(2(q+1))^{2}$, again a contradiction.

(2) Assume now that $(m, t)=(2,3)$. In particular, $H=\mathrm{A}_{3}$ or $\mathrm{S}_{3}$, and in the latter case $g$ is rational. Also, $g$ acts trivially on $W$. Hence by Lemma 5.4(iv) we have

$$
q^{4} \leq\left|V / C_{V}(g)\right|<\left|B: C_{B}(g)\right| \leq(2(q+1))^{2},
$$

a contradiction as $q \geq 3$. 


\subsubsection{The case $p=2$.}

Lemma 5.9. Assume $p=2$ and $t \geq 4$. Then $t=4$ and $m=q=2$, i.e. $V=\mathbb{F}_{2}^{16}$.

Proof Recall that $H$ is an even-order transitive subgroup of $\mathrm{S}_{t}$. We claim that $H$ contains an involution $h=d k$ with $d \in \mathrm{S}_{4}$ a double transposition and $k \in \mathrm{S}_{t-4}$ disjoint from $d$. (If not, then we may assume $H \ni x=(1, t)$. Since $H$ is transitive, for any $2 \leq i \leq t-1$ we can find $u \in H$ with $1^{u}=i$, and so $H \ni x^{u}=\left(i, t^{u}\right)$. If $t^{u} \neq 1, t$ then $H \ni x \cdot x^{u}=(1, t)\left(i, t^{u}\right)$, contrary to our assumption. If $t^{u}=t$, then $x^{u}=(i, t) \in H$. If $t^{u}=1$, then $H \ni x^{u x}=(i, t)$. We have shown that $(i, t) \in H$ for all $i$ with $1 \leq i \leq t-1$, and so $H=\mathrm{S}_{t} \ni(12)(34)$, again a contradiction.)

Without loss of generality we may now assume that $G$ contains an involution $h$ which permutes $V_{1}$ with $V_{2}, V_{3}$ with $V_{4}$, and acts on $V_{5}, \ldots, V_{t}$. Arguing as in the discussion about $g$ preceding Lemma 5.5, we see that

$$
\left(V_{1} \otimes V_{2}\right) \downarrow h=\left(V_{3} \otimes V_{4}\right) \downarrow h=\left(J_{1}^{a}, J_{2}^{b}\right) .
$$

Then setting $M:=V_{1} \otimes \ldots \otimes V_{4}$ and arguing as in the proof of Lemma 5.5 we obtain

$$
\gamma:=\frac{\operatorname{dim} C_{V}(h)}{\operatorname{dim} V} \leq \frac{\operatorname{dim} C_{M}(h)}{\operatorname{dim} M}=\frac{a^{2}+2 a b+2 b^{2}}{m^{4}}=\frac{1}{2}+\frac{1}{2 m^{2}} .
$$

In particular, $\gamma \leq 5 / 8$. Now we will apply Lemmas 5.4 and 5.6(i) to $h$ instead of $g$, and treat the cases described in Lemma 5.7 separately.

Assume first that $(m, t)=(2,6)$. Then $\left|C_{V}(h)\right| \leq|V|^{\gamma} \leq q^{40}$, so $\left|V / C_{V}(h)\right| \geq q^{24}$. On the other hand, by Lemmas 5.6 and 2.6(i), we have

$$
\frac{1}{2}\left|G: C_{B}(h)\right| \leq\left|G: C_{G}(h)\right| \leq 360 \cdot\left(q^{2}-1\right)^{6}<q^{24},
$$

contradicting Lemma 5.4(iv).

Next assume that $(m, t)=(2,5)$. Then $\left|C_{V}(h)\right| \leq|V|^{\gamma} \leq q^{20}$, so $\left|V / C_{V}(h)\right| \geq q^{12}$. Hence by Lemmas $5.4(\mathrm{iv})$ and 5.6 we must have

$$
q^{12}<\frac{1}{2}\left|G: C_{B}(h)\right| \leq\left|G: C_{G}(h)\right| \leq 60 \cdot\left(q^{2}-1\right)^{5},
$$

and so $q=2$ or 4 . If $q=4$, then by Lemmas 2.6 and 5.6 we have $\left|B: C_{B}(h)\right| \leq(q+1)^{5}$, whence

$$
\left|G: C_{G}(h)\right| \leq 60 \cdot(q+1)^{5}<q^{12},
$$

a contradiction. If $q=2$, then for $Q \in S y l_{2}(G)$ we have

$$
\left|G: N_{G}(Q)\right| \leq 15 \cdot 3^{5}<2^{12},
$$

again a contradiction by Lemma 5.4 (iii).

Finally, we assume that $(m, t)=(2,4)$ and $q \geq 4$. Then $\left|C_{V}(h)\right| \leq|V|^{\gamma} \leq q^{10}$. Also, any $2^{\prime}$-subgroup of $P G L_{2}(q)$ has order at most $q+1$ by Lemma $2.6(\mathrm{i})$, and $|\bar{H}| \leq\left|\mathrm{S}_{4}\right|$. In particular, the involution $h$ is central in some $Q \in \operatorname{Syl}_{2}(G)$. Furthermore, $C_{B}(h)$ has odd order, so $\left|C_{G}(h)\right| \geq|Q| \cdot\left|C_{B}(h)\right|$. It follows that

$$
\left|G: C_{G}(h)\right| \leq|H: Q| \cdot\left|B: C_{B}(h)\right| \leq 3 \cdot(q+1)^{4}<q^{6},
$$

a contradiction for $q \geq 4$.

Lemma 5.10. Suppose that $p=2$ and $t=3$. Then either $m=3$ and $q=2$, or $m=2$ and $q \leq 4$.

Proof By Lemma 5.7 we need to distinguish two cases.

(1) Assume that $(m, t)=(4,3)$. Then $\kappa \leq 5 / 8$ by Lemma 5.5 and so $\left|C_{V}(g)\right| \leq q^{40}$. Observe that any $2^{\prime}$-subgroup $X$ of $P G L_{4}(q) \cong S L_{4}(q)$ has order $\leq\left(q^{3}-1\right)\left(q^{2}-1\right)(q-1)$. (Indeed, this follows from Lemma 2.6 if $X$ acts reducibly on $V_{1}=\mathbb{F}_{q}^{4}$. Suppose that this action is irreducible. Then $\operatorname{Hom}_{X}\left(V_{1}\right) \cong \mathbb{F}_{q^{a}}$ for some $a \mid 4$, and $V_{1}$ is a $(4 / a)$-dimensional absolutely irreducible $\mathbb{F}_{q^{a}} X$-module. Since $X$ is soluble, any irreducible Brauer character of $X$ lifts to a complex character by the Fong-Swan Theorem [12, 72.1]; in particular, 4/a 
divides $|X|$ and so $a=4$. This in turn implies that $X \leq G L_{1}\left(q^{4}\right)$, and so we are done.) Hence, applying Lemma 5.6 to the element $g$ defined before Lemma 5.5, we have

$$
q^{24}<\left|G: C_{G}(g)\right| \leq 3 \cdot\left(\left(q^{3}-1\right)\left(q^{2}-1\right)(q-1)\right)^{3},
$$

a contradiction.

(2) Consider the case $(m, t)=(3,3)$ and $q \geq 4$. Note that any $2^{\prime}$-subgroup of $P G L_{3}(q)$ has order $\leq q^{3}-1$ by Lemma 2.6(ii). Assume in addition that the involution $g$ acts nontrivially on $V_{3}=\mathbb{F}_{q}^{3}$, i.e. $V_{3} \downarrow g=\left(J_{1}, J_{2}\right)$. Then

$$
V \downarrow g=\left(J_{1}^{3}, J_{2}^{3}\right) \otimes\left(J_{1}, J_{2}\right)=\left(J_{1}^{3}, J_{2}^{12}\right)
$$

and so $\left|C_{V}(g)\right|=q^{15}$. Lemmas 5.4(iv) and 5.6 now imply that

$$
q^{12}<\left|G: C_{G}(g)\right| \leq 3 \cdot\left(q^{3}-1\right)^{3},
$$

a contradiction.

Thus $g$ acts trivially on $V_{3}=W$. In this case, by Lemma 5.6(ii) we have

$$
q^{9} \leq\left|V / C_{V}(g)\right|<\left|G: C_{G}(g)\right| \leq 3 \cdot\left(q^{3}-1\right),
$$

again a contradiction.

(3) Now assume $(m, t)=(2,3)$ and $q \geq 8$. Note that any $2^{\prime}$-subgroup of $P G L_{2}(q)$ has order $\leq q+1$ by Lemma 2.6(i). Assume in addition that the involution $g$ acts nontrivially on $V_{3}=\mathbb{F}_{q}^{2}$, i.e. $V_{3} \downarrow g=J_{2}$. Then

$$
V \downarrow g=\left(J_{1}^{2}, J_{2}\right) \otimes J_{2}=J_{2}^{4}
$$

and so $\left|C_{V}(g)\right|=q^{4}$. Lemmas 5.4(iv) and 5.6 now imply that

$$
q^{4}<\left|G: C_{G}(g)\right| \leq 3 \cdot(q+1)^{3},
$$

a contradiction. Thus $g$ acts trivially on $V_{3}=W$. In this case, by Lemma 5.6(ii) we have

$$
q^{2} \leq\left|V / C_{V}(g)\right|<\left|G: C_{G}(g)\right| \leq 3 \cdot(q+1),
$$

again a contradiction.

The rest of this subsection is devoted to the case $t=2$, so $V=V_{1} \otimes V_{2}$ and $p=2$ by Lemma 5.4(ii), and $V_{1}, V_{2}$ are interchanged by $g$. As before, we fix the basis $\left(e_{j}:=e_{j}^{1} \mid 1 \leq j \leq m\right)$ of $V_{1}$ and $\left(f_{j}:=e_{j}^{2}=e_{j} g \mid 1 \leq j \leq m\right)$ of $V_{2}$. Then we can identify both $V_{1}$ and $V_{2}$ with $\mathbb{F}_{q}^{m}$. Consider the subgroups $Y_{1} \cong Y_{2}$ of $G L_{m}(q)$ defined in (1).

The key observation in the case $t=2$ is the following:

Lemma 5.11. Suppose $t=2$. Then the subgroup $Y_{1}<G L_{m}(q)$ is transitive on $k$ dimensional subspaces of $\mathbb{F}_{q}^{m}$ for any $k \leq m-1$.

Proof Recall that if $0 \neq v \in V$ has weight $k: v=\sum_{i=1}^{k} x_{i} \otimes y_{i}$, then

$$
[v]_{1}:=\left\langle x_{1}, \ldots, x_{k}\right\rangle_{\mathbb{F}_{q}},[v]_{2}:=\left\langle y_{1}, \ldots, y_{k}\right\rangle_{\mathbb{F}_{q}}
$$

are $k$-dimensional subspaces of $\mathbb{F}_{q}^{m}$ uniquely determined by $v$. In particular, if $v g=v$, then

$$
\sum_{i=1}^{k} x_{i} \otimes y_{i}=v=v g=\sum_{i=1}^{k} y_{i} \otimes x_{i},
$$

and so $\left([v]_{1}\right) g=[v]_{2}$.

Next we show that if $0 \neq v \in V$ has weight $k$ and is fixed by some involution $g^{\prime}$ of $G$, then there is some element $a \otimes b \in B$ such that $[v]_{2} b=\left([v]_{1} a\right) g$. Indeed, since $|G|=2|B|$ and $|B|$ is odd, we must have $g^{\prime}=x g x^{-1}$ for some $x=a \otimes b \in B$. Writing $v=\sum_{i=1}^{k} x_{i} \otimes y_{i}$, we see that $w:=v x=\sum_{i=1}^{k} x_{i} a \otimes y_{i} b$ is fixed by $g$. Hence, according to the first paragraph we then have

as stated.

$$
\left([v]_{1} a\right) g=\left([w]_{1}\right) g=[w]_{2}=\left([v]_{2}\right) b,
$$


Now we set $L:=\left\langle e_{1}, \ldots, e_{k}\right\rangle_{\mathbb{F}_{q}}, M:=\left\langle f_{1}, \ldots, f_{k}\right\rangle_{\mathbb{F}_{q}}$, and consider any $k$-dimensional subspace $N:=\left\langle u_{1}, \ldots, u_{k}\right\rangle_{\mathbb{F}_{q}}$ of $V_{1}$. Then $v=\sum_{i=1}^{k} u_{i} \otimes f_{i} \in V$ has weight $k$, with $[v]_{1}=N,[v]_{2}=M$. The $p$-exceptionality of $G$ implies that $v$ is fixed by some involution $g^{\prime} \in G$. By the previous paragraph, there is some $x=a \otimes b \in B$ such that

$$
(N a) g=\left([v]_{1} a\right) g=\left([v]_{2}\right) b=M b=(L g) b,
$$

i.e. $N a=L g b g^{-1}$. Writing $f_{j} b=\sum_{i=1}^{m} b_{i j} f_{i}$ for some $b_{i j} \in \mathbb{F}_{q}$, we have $\left(e_{j}\right) g b g^{-1}=$ $\sum_{i=1}^{m} b_{i j} e_{i}$. Thus, under our identification of $V_{1}$ and $V_{2}$ with $\mathbb{F}_{q}^{m}$, we have $N a=L g b g^{-1}=$ $L b$, and so $L b a^{-1}=N$. It remains to observe that $B$ contains $x^{g} x^{-1}=\left(b a^{-1}\right) \otimes\left(a b^{-1}\right)$, whence $b a^{-1} \in Y_{1}$.

Proposition 5.12. Assume $t=2$ ( so $p=2)$. Then either $m=2$, or $m=3$ and $q \leq 8$.

Proof By Lemma 5.11, $Y_{1}$ is transitive on $k$-spaces for all $k$, and has odd order. Hence [31, Lemma 4.1] shows that if $m>2$ then either $m=3$ or $(m, q)=(5,2)$. In the latter case $Y_{1}=\Gamma L_{1}(32), V=\mathbb{F}_{2}^{25}$, and $\left|C_{V}(g)\right| \leq 2^{15}$ by Lemma 5.5. Applying Lemma 5.4(iv), $\left|B: C_{B}(g)\right|>2^{10}$. On the other hand, the proof of Lemma 5.6(ii) shows that $\left|B: C_{B}(g)\right| \leq$ $\left|Y_{1}\right|=155$, a contradiction

Next suppose that $m=3$. By Lemma 5.5 and Lemma 5.4(iv) we have $\left|B: C_{B}(g)\right|>$ $\left|V / C_{V}(g)\right| \geq q^{3}$. Now the proof of Lemma 5.6(ii) shows that $q^{3}<\left|B: C_{B}(g)\right| \leq\left|Y_{1}\right| /(q-$ 1) $\leq 3 f\left(q^{2}+q+1\right)$, which can happen only when $q \leq 8$.

This completes the proof of Theorem 5.1.

5.2. Proof of Theorem 5.2. Throughout this section we assume that $G \leq \Gamma L(V)$ is a $p$-exceptional group such that $G_{0} \triangleleft G=\left\langle G_{0}, \sigma\right\rangle$, where

(i) $G_{0}$ is an absolutely irreducible $p^{\prime}$-subgroup of $G L(V)$ which preserves a tensor-induced decomposition

$$
V=\left(V_{1}\right)^{\otimes t}=V_{1} \otimes V_{2} \otimes \ldots \otimes V_{t},
$$

with $V=\mathbb{F}_{q}^{n}, \operatorname{dim}_{\mathbb{F}_{q}} V_{i}=m \geq 2, t \geq 2$, and

(ii) $q=q_{0}^{p}, q_{0}=p^{f}$, and $\sigma$ induces the field automorphism $x \mapsto x^{q_{0}}$ of $V$ modulo $G L(V)$. Note that $\left|C_{V}(\sigma)\right| \leq|V|^{1 / p}$. Indeed, since $\mathcal{G}:=G L\left(V \otimes_{\mathbb{F}_{q}} \overline{\mathbb{F}}_{q}\right)$ is connected (where $\overline{\mathbb{F}}_{q}$ is the algebraic closure of $\left.\mathbb{F}_{q}\right), \sigma$ is $\mathcal{G}$-conjugate to the standard Frobenius morphism $\sigma_{0}: x \mapsto x^{q_{0}}$. Hence

$$
\left|C_{V}(\sigma)\right| \leq\left|C_{\left(V \otimes_{\mathbb{F}_{q}} \overline{\mathbb{F}}_{q}\right)}(\sigma)\right|=\left|C_{\left(V \otimes_{\mathbb{F}_{q}} \overline{\mathbb{F}}_{q}\right)}\left(\sigma_{0}\right)\right|=|V|^{1 / p}
$$

5.2.1. First reductions. The following lemma simplifies further computations.

Lemma 5.13. Under the above assumptions,

(i) $m^{t}$ divides $\left|G_{0}\right|$; in particular, $p$ does not divide $m$, and

(ii) $\left|G_{0}\right|>|V|^{1-1 / p}$.

Proof Part (i) follows from the assumptions that $G_{0}$ is a $p^{\prime}$-group and absolutely irreducible. For (ii), one can argue as in the proof of Lemma 5.4(iii), taking $Q=\langle\sigma\rangle$.

Next we rule out most of the cases using Lemma 5.13:

Proposition 5.14. Under the above assumptions, $t=2$.

Proof Assume to the contrary that $t \geq 3$. The proof of Lemma 5.6 implies that the base subgroup $G_{0} \cap\left(G L\left(V_{1}\right) \circ \ldots \circ G L\left(V_{t}\right)\right)$ of $G_{0}$ has order at most $(q-1) \cdot|X|^{t}$, where $X$ is a $p^{\prime}$-subgroup of largest possible order of $P G L_{m}(q)$. Hence

$$
\left|G_{0}\right| \leq t ! \cdot(q-1) \cdot\left(\left|P G L_{m}(q)\right|_{p^{\prime}}\right)^{t}<\left(\frac{t+1}{2}\right)^{t} \cdot q^{t\left(\frac{m(m+1)}{2}-1\right)+1}
$$


and Lemma 5.13(ii) yields that

$$
f(m, t, p):=m^{t}\left(1-\frac{1}{p}\right)-t \cdot \frac{m^{2}+m-2}{2}-1-t \cdot \log _{p} \frac{t+1}{2}<0 .
$$

Note that the function $f(m, t, p)$ is non-decreasing for each of its variables. Now direct computations show that the latter inequality is impossible unless one of the following holds.

(a) $t=5, m=p=2$. This is ruled out by Lemma $5.13(\mathrm{i})$.

(b) $t=4, m=2, p \leq 3$. By Lemma 5.13(i) we must have $p=3$ and so $q=q_{o}^{p} \geq 27$. Since for $m=2$ we have $|X| \leq 2(q+1)$ by Lemma 2.6(i),

$$
\left|G_{0}\right| \leq 24 \cdot(2(q+1))^{4} \cdot(q-1)<q^{32 / 3}=|V|^{2 / 3},
$$

a contradiction by Lemma 5.13(ii).

(c) $(t, m, p)=(3,3,2)$. In this case $q \geq 4$, and $|X| \leq q^{3}-1$ as $m=3$ by Lemma 2.6(ii). It follows that

again contradicting Lemma $5.13(\mathrm{ii})$.

$$
\left.\left|G_{0}\right| \leq 6 \cdot\left(q^{3}-1\right)\right)^{3} \cdot(q-1)<q^{27 / 2}=|V|^{1 / 2},
$$

(d) $(t, m)=(3,2)$. By Lemma $5.13(\mathrm{i})$ we must have $p \geq 3$ and so $q \geq 27$. Also, by Lemma 2.6(i) we have $|X| \leq 2(q+1)$ as $m=2$. Hence

$$
\left|G_{0}\right| \leq 6 \cdot(2(q+1))^{3} \cdot(q-1)<q^{16 / 3} \leq|V|^{1-1 / p},
$$

again a contradiction.

5.2.2. The case $t=2$. Throughout this subsection we assume $t=2$.

Lemma 5.15. We have $G_{0} \leq G L\left(V_{1}\right) \circ G L\left(V_{2}\right)$ and $p=2$.

Proof Let $B=G_{0} \cap\left(G L\left(V_{1}\right) \circ G L\left(V_{2}\right)\right)$, and suppose $G_{0} \neq B$. Then $G_{0}=B\langle s\rangle=B .2$, where $s$ interchanges $V_{1}$ and $V_{2}$. Since $G_{0}$ is a $p^{\prime}$-group this implies that $p>2$. As $\sigma$ has order $p$ it therefore fixes $V_{1}$ and $V_{2}$, and so $B\langle\sigma\rangle$ is a normal subgroup of index 2 in $G$, hence is $p$-exceptional. This contradicts Theorem 4.2 .

Hence $G_{0}=B$. If $p>2$ then again $\sigma$ fixes $V_{1}, V_{2}$ and we contradict Theorem 4.2. Hence $p=2$, completing the proof.

Proposition 5.16. The case $t=p=2$ cannot occur.

Proof Assume to the contrary that $t=p=2$. By Lemma 5.15 we have $G_{0}=B$ and $G=B\langle\sigma\rangle$ with $\sigma$ of order 2 , and $G_{0}$ is an absolutely irreducible $2^{\prime}$-group on $V$. By Lemma $5.13, m$ is odd; in particular, $m \geq 3$. If $\sigma$ fixes both $V_{1}$ and $V_{2}$, then Theorem 4.2 gives a contradiction. So $\sigma$ interchanges $V_{1}$ and $V_{2}$ and also it is semilinear: $(\lambda v)^{\sigma}=\lambda^{r} v$ with $q=2^{2 f}=r^{2}$.

We will now follow the arguments in Subsection 5.1.3 for the corresponding case in Theorem 4.1, and indicate necessary modifications because of the semilinearity of $\sigma$. We fix the basis $\left(e_{j}:=e_{j}^{1} \mid 1 \leq j \leq m\right)$ of $V_{1}$ and $\left(f_{j}:=e_{j}^{2}=\sigma\left(e_{j}\right) \mid 1 \leq j \leq m\right)$ of $V_{2}$. Then we can identify both $V_{1}$ and $V_{2}$ with $\mathbb{F}_{q}^{m}$. Consider the subgroups $Y_{1} \cong Y_{2}$ of $G L_{m}(q)$ defined in (1). Note that if $x=X \otimes Y \in B$, then $x^{\sigma}=Y^{(r)} \otimes X^{(r)}$, where $X^{(r)}=\left(x_{i j}^{r}\right)$ if $X=\left(x_{i j}\right)$. In particular, if $X \in Y_{1}$ then $X^{(r)} \in Y_{2}$ and vice versa, whence $Y_{1} \cong Y_{2}$. Now the proof of Lemma 5.11 can be carried over verbatim, except that we have to replace $b a^{-1}$ by $b^{(r)} a^{-1}$. Thus $Y_{1}$ (and $Y_{2}$ ) is transitive on $k$-spaces of $\mathbb{F}_{q}^{m}$ for all $k$, and $\left|Y_{1}\right|$ is odd (and contains all scalar matrices). Now [31, Lemma 4.1] implies that $m=3$ or $(m, q)=(5,2)$. The latter is impossible as $q=r^{2}$.

Hence $m=3$. Now we consider the homomorphism $f^{*}: Y_{1} \times Y_{2} \rightarrow Y_{1} \otimes Y_{2}$ defined by $f^{*}(X, Y)=X \otimes Y$, and the subgroup $K:=\left\{\left(X, X^{(r)}\right) \mid X \in Y_{1}\right\}$ of $Y_{1} \times Y_{2}$. Note that $\sigma$ centralizes $f^{*}(K)$. As in the proof of Lemma 5.6(ii), this implies that $\left|B: C_{B}(\sigma)\right|$ has order at most $\left|\left(Y_{1} \times Y_{2}\right): K\right|=\left|Y_{1}\right|$. On the other hand, $\left|C_{V}(\sigma)\right|=|V|^{1 / 2}=q^{9 / 2}$. Hence the 2-exceptionality of $G$ implies $\left|G: C_{G}(\sigma)\right| \geq\left|V / C_{V}(\sigma)\right|=q^{9 / 2}$ by Lemma 2.4(ii). Since $\left|C_{G}(\sigma)\right|=2\left|C_{B}(\sigma)\right|$, we get

$$
q^{9 / 2} \leq\left|Y_{1}\right| \leq 6 f\left(q^{3}-1\right)
$$


again a contradiction.

This completes the proof of Theorem 5.2.

5.3. Proof of Proposition 5.3. Now we prove Proposition 5.3 by treating the cases left in conclusions (i)-(iii) of Theorem 5.1. Let $G$ be as in the hypothesis of the proposition. If $(m, t) \neq(2,2)$ then there are just a small number of cases for $(m, t, q)$ to consider, all with $q \leq 8$ and $m^{t} \leq 16$, and a MAGMA computation shows that the only irreducible $p$-exceptional examples are those in part (a) of Proposition 5.3. (For $(m, t)=(3,2)$ and $q=4,8$ we first use Lemmas $5.4(\mathrm{i})$ and 5.11 to reduce to $G$ being a subgroup of $\left(\Gamma L_{1}\left(q^{m}\right) \circ \Gamma L_{1}\left(q^{m}\right)\right) .2$ before doing the MAGMA computations.)

The remaining case $(m, t)=(2,2)$ is handled by the following result.

Proposition 5.17. Suppose $G<G L(V)$ is an irreducible p-exceptional subgroup satisfying the assumptions of Theorem 5.1 with $m=t=p=2$. Then $G$ is conjugate to a subgroup of $G L_{2}\left(q^{2}\right)$ (and so is known by Lemma 2.8).

Proof Without loss of generality, we may assume that $G$ contains $Z=Z(G L(V))$. Consider the base subgroup $B=G \cap\left(G L\left(V_{1}\right) \circ G L\left(V_{2}\right)\right)$ of index at most 2 in $G$. Then all $B$-orbits on $V$ have odd lengths. Hence, by Theorem 4.1, either $|B|$ is odd, or $B$ is (conjugate to) the group $H$ appearing in the conclusion of that theorem.

Consider the latter case. We may identify $B$ with a subgroup of index 2 in the group $\tilde{C}$ defined in the proof of Lemma 4.9. Adopt the notation of that proof. Since $G$ normalizes $C=O_{2^{\prime}}(B), G$ permutes the two $C$-orbits $U_{1}^{\sharp}$ and $U_{2}^{\sharp}$ of length $q^{2}-1$. Since $G$ is irreducible, $G$ cannot fix either of them. Thus $G$ interchanges them, and so $G$ has an orbit of length $2\left(q^{2}-1\right)$, contradicting the 2-exceptionality of $G$.

Hence $|B|$ is odd. Thus $|G|$ is not divisible by 4 and so $G$ is soluble. Also $G$ is irreducible on $V$. Hence by the Fong-Swan theorem, the dimension $d$ of $V$ over $\operatorname{End}_{G}(V) \supseteq \mathbb{F}_{q}$ cannot be 4 (but divides 4 ), and so it is either 1 or 2 . If $d=1$, then $|G|$ divides $\left|G L_{1}\left(q^{4}\right)\right|$ and so it is odd, a contradiction. Thus $d=2$ and $G \leq G L_{2}\left(q^{2}\right)$ as in the conclusion.

This completes the proof of Proposition 5.3.

\section{SubFiELDS}

It turns out that a $p$-exceptional group $H \leq \Gamma L_{n}(q)$ cannot be realisable modulo scalars over a proper subfield $\mathbb{F}_{q_{0}}$ of $\mathbb{F}_{q}$. Such groups are conjugate to subgroups of $Z \circ G L_{n}\left(q_{0}\right) \cdot\langle\phi\rangle$, for some proper subfield $\mathbb{F}_{q_{0}}$ of $\mathbb{F}_{q}$, where $\phi$ generates the group of field automorphisms of $G L_{n}(q)$ and $Z=Z\left(G L_{n}(q)\right)$. We prove

Theorem 6.1. Let $V=\mathbb{F}_{q}^{n}$, and $q=q_{0}^{s}$ with $s>1$. Suppose $H \leq\left(Z \circ G L_{n}\left(q_{0}\right)\right)\langle\phi\rangle<$ $\Gamma L(V)$, where $\phi$ generates the group of field automorphisms of $G L_{n}(q)$. Then $H$ is not p-exceptional.

Proof Suppose that $H$ is $p$-exceptional on $V=\mathbb{F}_{q}^{n}$. By Lemma 2.1, $Z H$ is also $p$ exceptional, so we may assume that $Z \subseteq H$. Then $H \cap G L_{n}(q)=Z \circ H_{0}$, where $H_{0}=$ $H \cap G L_{n}\left(q_{0}\right)$, and note that $H_{0}$ is normal in $H$. Let $\left\{b_{1} \ldots, b_{s}\right\}$ be a basis for $\mathbb{F}_{q}$ as an $\mathbb{F}_{q_{0}}$-vector space. Then each $v \in V$ may be written as a sum $\sum_{i=1}^{s} b_{i} v_{i}$ with $v_{i} \in V_{0}=\mathbb{F}_{q_{0}}^{n}$. If the non-zero $v_{i}$ are linearly independent in $V_{0}$ then the stabiliser $\left(H_{0}\right)_{v}$ must fix each of the $v_{i}$.

Suppose first that $p$ divides $\left|H_{0}\right|$, or equivalently that $p$ divides $\left|H \cap G L_{n}(q)\right|$. Then $H_{0}$ is $p$-exceptional on $V$, and hence also on $V_{0}$, by Lemma 2.1. Let $x \in H_{0}$ have order $p$. Then there exists a 2-dimensional $x$-invariant $\mathbb{F}_{q_{0}}$-subspace $\left\langle v_{1}, v_{2}\right\rangle_{\mathbb{F}_{q_{0}}}$ of $V_{0}$ which is not fixed pointwise by $x$. Let $v=b_{1} v_{1}+b_{2} v_{2}$. Then as we observed in the previous paragraph, $\left(H_{0}\right)_{v}$ fixes both $v_{1}$ and $v_{2}$ and hence leaves the $\mathbb{F}_{q}$-space $U:=\left\langle v_{1}, v_{2}\right\rangle_{\mathbb{F}_{q}}$ invariant, fixing it 
pointwise. Moreover $\left(H_{0}\right)_{v}=\left(H_{0}\right)_{v_{1}, v_{2}}$ is the kernel of the action of $\left(H_{0}\right)_{U}$ on $U$. It follows that $x \in\left(H_{0}\right)_{U} \backslash\left(H_{0}\right)_{v}$ and hence that $p$ divides $\left|\left(H_{0}\right)_{U}:\left(H_{0}\right)_{v}\right|$, which divides $\left|H_{0}:\left(H_{0}\right)_{v}\right|$, a contradiction to $p$-exceptionality.

Thus $\left|H_{0}\right|$ is coprime to $p$, and $H$ has a normal subgroup which contains $H \cap G L_{n}(q)$ as a subgroup of index $p$. By Lemma 2.1, this normal subgroup is $p$-exceptional and so, without loss of generality, we may assume that $H=\left(H \cap G L_{n}(q)\right) \cdot\langle x\rangle=\left(Z \circ H_{0}\right) \cdot\langle x\rangle$, where $x$ is a field automorphism of order $p$, and so $x$ induces a (possibly trivial) field automorphism on $G L_{n}\left(q_{0}\right)$. Thus each element of $H$ has the form $x^{i} c h$, for some $i$, with $c \in Z$ and $h=\left(a_{i j}\right) \in G L_{n}\left(q_{0}\right)$. Recall that $q=q_{0}^{s}$, and note that $x$ fixes pointwise (at least) an $\mathbb{F}_{p}$-subspace $\mathbb{F}_{p}^{n}$ of $V_{0}$. Let $q_{1}:=q^{1 / p}$, so $\mathbb{F}_{q_{1}}$ is the fixed field of $x$.

Choose $v_{1}, v_{2}$ linearly independent vectors in $V_{0}$ fixed by $x$, let $U:=\left\langle v_{1}, v_{2}\right\rangle_{\mathbb{F}_{q}}$, and define

$$
X(U):=\left\{c_{1} v_{1}+c_{2} v_{2} \mid c_{1}, c_{2} \in \mathbb{F}_{q}^{*}, c_{1} c_{2}^{-1} \notin \mathbb{F}_{q_{0}}\right\} .
$$

First we show that (i) for $v \in X(U)$, the stabiliser $H_{v} \subseteq H_{U}$, and (ii) $X(U)$ is $H_{U}$-invariant. Let $v=c_{1} v_{1}+c_{2} v_{2} \in X(U)$ and extend $\left\{v_{1}, v_{2}\right\}$ to a basis $\left\{v_{1}, v_{2}, \ldots, v_{n}\right\}$ for $V$ over $\mathbb{F}_{q}$.

(i) Let $x^{i} c h \in H_{v}$ with $c, h$ as in the previous paragraph, with $h$ represented with respect to the basis $\left\{v_{1}, \ldots, v_{n}\right\}$. Since $x$ and $Z$ leave $U$ invariant it is sufficient to show that $h$ does also. Now

$$
v=v^{x^{i} c h}=c \sum_{j=1}^{n}\left(c_{1}^{x^{i}} a_{1 j}+c_{2}^{x^{i}} a_{2 j}\right) v_{j}
$$

and so, for each $j \geq 3, c_{1}^{x^{i}} a_{1 j}+c_{2}^{x^{i}} a_{2 j}=0$ which implies that $\left(c_{1} c_{2}^{-1}\right)^{x^{i}} a_{1 j}=-a_{2 j}$. Since $a_{1 j}, a_{2 j} \in \mathbb{F}_{q_{0}}$ while $\left(c_{1} c_{2}^{-1}\right)^{x^{i}} \notin \mathbb{F}_{q_{0}}$, it follows that $a_{1 j}=a_{2 j}=0$. Thus $h$ leaves $U$ invariant, proving part (i). Also we have, for $j=1,2$, that $c_{j}=c\left(c_{1}^{x^{i}} a_{1 j}+c_{2}^{x^{i}} a_{2 j}\right)$, whence

$$
c^{-1}=c_{1}^{-1} c_{1}^{x^{i}} a_{11}+c_{1}^{-1} c_{2}^{x^{i}} a_{21}=c_{2}^{-1} c_{1}^{x^{i}} a_{12}+c_{2}^{-1} c_{2}^{x^{i}} a_{22}
$$

and since $h$ is nonsingular, $a_{11} a_{22}-a_{12} a_{21} \neq 0$.

(ii) Since $c_{1}^{x^{i}}\left(c_{2}^{x^{i}}\right)^{-1}=\left(c_{1} c_{2}^{-1}\right)^{x^{i}} \notin \mathbb{F}_{q_{0}}$, and since $\left(c c_{1}\right)\left(c c_{2}\right)^{-1}=c_{1} c_{2}^{-1} \notin \mathbb{F}_{q_{0}}$, it follows that $x^{i}$ and each element of $Z$ leaves $X(U)$ invariant. It remains to consider $v^{h}$ where $h=\left(a_{i j}\right) \in H_{U}$. Now

$$
v^{h}=\left(c_{1} a_{11}+c_{2} a_{21}\right) v_{1}+\left(c_{1} a_{12}+c_{2} a_{22}\right) v_{2} .
$$

If the coefficient of $v_{2}$ were 0 we would have $a_{22}=-a_{12}\left(c_{1} c_{2}^{-1}\right) \in \mathbb{F}_{q_{0}}$ and hence $a_{22}=$ $a_{12}=0$. However $a_{1 j}=a_{2 j}=0$ for each $j \geq 3$, and this would imply that $h$ is singular, a contradiction. A similar argument shows that the coefficient of $v_{1}$ is also nonzero. Suppose for a contradiction that $v^{h} \notin X(U)$. Then

$$
d:=\left(c_{1} a_{11}+c_{2} a_{21}\right)\left(c_{1} a_{12}+c_{2} a_{22}\right)^{-1} \in \mathbb{F}_{q_{0}}^{*}
$$

and we have $c_{1}\left(a_{11}-d a_{12}\right)=c_{2}\left(d a_{22}-a_{21}\right)$. If $d a_{22}-a_{21} \neq 0$ then $c_{2} c_{1}^{-1}=\left(d a_{22}-\right.$ $\left.a_{21}\right)^{-1}\left(a_{11}-d a_{12}\right) \in \mathbb{F}_{q_{0}}$ which is a contradiction. Thus $a_{21}=d a_{22}$, and hence also $a_{11}=$ $d a_{12}$. This again implies that $h$ is singular, and finally we conclude that $v^{h} \in X(U)$, proving (ii).

We have $\langle x\rangle \leq H_{U}$, and since the $p$-part of $|H|$ is $p$, it follows that $\langle x\rangle$ is a Sylow $p$ subgroup of $H_{U}$. By $p$-exceptionality, $\left|H_{v}\right|$ is divisible by $p$ and hence, by Sylow's Theorem, it follows that $x$ must fix at least one vector of $X(U)$. Without loss of generality we may assume that $x$ fixes $v$. Now observe that $v=v^{x}$ if and only if $c_{j}=c_{j}^{x}$ for $j=1,2$, and hence $\left(c_{1} c_{2}^{-1}\right)^{x}=c_{1} c_{2}^{-1} \in \mathbb{F}_{q_{1}} \backslash \mathbb{F}_{q_{0}}$. Thus $\mathbb{F}_{q_{1}} \nsubseteq \mathbb{F}_{q_{0}}$.

Now we consider a special element of $X(U)$, namely $v^{\prime}=v_{1}+b v_{2}$, where $b$ is a primitive element of $\mathbb{F}_{q_{1}}$ (which we have just shown does not lie in $\mathbb{F}_{q_{0}}$ ). Suppose that $x^{i} c h$ fixes $v^{\prime}$, and note that $b^{x^{i}}=b$ since $b$ lies in the fixed field $\mathbb{F}_{q_{1}}$ of $x$. Then equation (2) in the computation in (i) shows that

$$
c^{-1}=a_{11}+b a_{21}=b^{-1} a_{12}+a_{22}
$$

which implies that $b^{2} a_{21}+b\left(a_{11}-a_{22}\right)-a_{12}=0$. Since $b \notin \mathbb{F}_{q_{0}}$, it follows that $b$ has minimal polynomial over $\mathbb{F}_{q_{0}}$ of degree 2 . The extension field $\mathbb{F}_{q_{0}}(b)$ contains the maximal subfield 
TABLE 1

\begin{tabular}{|l|l|l|l|}
\hline$R$ & $d$ & $N_{G L(V)}(R) / R Z$ & $q$ \\
\hline$r^{1+2 m}, r$ odd & $r^{m}$ & $S p_{2 m}(r)$ & $q \equiv 1 \bmod r$ \\
$4 \circ 2^{1+2 m}$ & $2^{m}$ & $S p_{2 m}(2)$ & $q \equiv 1 \bmod 4$ \\
$2_{ \pm}^{1+2 m}$ & $2^{m}$ & $O_{2 m}^{ \pm}(2)$ & $q \equiv 1 \bmod 2$ \\
\hline
\end{tabular}

$\mathbb{F}_{q_{1}}$ of $\mathbb{F}_{q}$ as a proper subfield, and hence $\mathbb{F}_{q_{0}}(b)=\mathbb{F}_{q}$ and $s=2$. Also, since $\mathbb{F}_{q_{1}} \nsubseteq \mathbb{F}_{q_{0}}, p$ is odd.

We may therefore write $q=r^{2 p}, q_{0}=r^{p}, q_{1}=r^{2}$, and then $\mathbb{F}_{q_{1}} \cap \mathbb{F}_{q_{0}}=\mathbb{F}_{r}$. Now $|X(U)|=$ $(q-1)\left(q-q_{0}\right)=r^{p}\left(r^{2 p}-1\right)\left(r^{p}-1\right)$. We showed above that the vectors of $X(U)$ fixed by $x$ are $c_{1} v_{1}+c_{2} v_{2}$ with each $c_{i} \in \mathbb{F}_{q_{1}}$ but $c_{1} c_{2}^{-1} \notin \mathbb{F}_{q_{0}}$. Thus $x$ fixes precisely $\left(r^{2}-1\right)\left(r^{2}-r\right)$ vectors in $X(U)$. Since each Sylow $p$-subgroup of $H_{U}$ fixes the same number of vectors in $X(U)$, the number of Sylow $p$-subgroups of $H_{U}$ is at least $\frac{r^{p}\left(r^{2 p}-1\right)\left(r^{p}-1\right)}{r\left(r^{2}-1\right)(r-1)}>r^{p-1} r^{2 p-2} r^{p-1}=r^{4(p-1)}$.

We now consider the induced group $H_{U}^{U} \leq \Gamma L(U)$. Since $|H \cap G L(V)|$ is coprime to $p$, and since $x$ acts nontrivially on $U$, it follows that $H_{U}^{U}$ is of the form $\left(Z^{U} \circ L\right) \cdot\left\langle x^{U}\right\rangle$ with $L$ a $p^{\prime}$-subgroup of $G L_{2}\left(q_{0}\right)$, where $Z^{U}$ is the group induced by $Z$. Note that the normaliser $N$ of $\left\langle x^{U}\right\rangle$ in $H_{U}^{U}$ intersects $Z^{U}$ in a subgroup of order $r^{2}-1$. Thus $\left|H_{U}^{U}: N Z^{U}\right|=$ $\left|H_{U}^{U}: N\right| /\left(\frac{r^{2 p}-1}{r^{2}-1}\right)$, which is at least $\frac{r^{p}\left(r^{p}-1\right)}{r(r-1)}>r^{2 p-2}$. Since $N Z^{U}$ contains $Z^{U} \cdot\left\langle x^{U}\right\rangle$, we have $\left|L /\left(L \cap Z^{U}\right)\right| \geq\left|H_{U}^{U}: N Z^{U}\right|>r^{2 p-2} \geq 3^{4}$. It then follows from the classification of subgroups of $P G L_{2}\left(q_{0}\right)$, that the $p^{\prime}$-group $L /\left(L \cap Z^{U}\right)$ is contained in a dihedral group $D_{2\left(q_{0}-1\right)}$ or $D_{2\left(q_{0}+1\right)}$. The number of Sylow $p$-subgroups of $H_{U}^{U}$ in the case where $L /\left(L \cap Z^{U}\right) \leq D_{2\left(q_{0} \pm 1\right)}$ is at most the number if equality holds. In the case of equality the normaliser of $\left\langle x^{U}\right\rangle$ in $H_{U}^{U}$ is $Z_{r^{2}-1} \cdot D_{2(r \pm 1)} \cdot\left\langle x^{U}\right\rangle$, and so the number of Sylow $p$-subgroups is $\frac{\left(r^{2 p}-1\right)\left(r^{p} \pm 1\right)}{\left(r^{2}-1\right)(r \pm 1)}<4 r^{3(p-1)}$, which is less than the lower bound $r^{4(p-1)}$. This contradiction completes the proof.

\section{Extraspecial type nORMALIZERs: $\mathcal{C}_{6}$ SUbGroups}

Let $r$ be a prime, $m$ a positive integer, and let $R$ be an $r$-group of symplectic type such that $|R / Z(R)|=r^{2 m}, R$ is of exponent $r \cdot(2, r)$, and $R$ is as in Table 1 . Let $V=V_{d}(q)$ be a faithful, absolutely irreducible $\mathbb{F}_{q} R$-module, where $r$ does not divide $q$. Then $d=\operatorname{dim} V=r^{m}$, and $N_{G L(V)}(R)$ is as in the table. Assume further that $R$ is not realised over a proper subfield of $\mathbb{F}_{q}$. Then $q$ is a minimal power of the characteristic $p$, subject to the conditions in the last column of the table.

Here we prove

Theorem 7.1. Let $r$ be a prime, and assume that $R$ and $V=V_{d}(q)$ are as above. Suppose $R \triangleleft G \leq N_{\Gamma L(V)}(R)$ and $G$ is p-exceptional, and is not transitive on $V^{\sharp}$. Then $G$ is imprimitive on $V$, and one of the following holds.

: (i) $r=2, q=3, d=4$ and $G=2^{1+4} \mathrm{~A}_{4}$ or $2^{1+4} \mathrm{~S}_{4}$, with orbits on vectors of sizes $1,16,64$.

: (ii) $r=3, q=4, d=3$ : here there are five 2-exceptional groups of the form $3^{1+2} . X$; they are $3^{1+2} .2,3^{1+2} .6,3^{1+2} \cdot S_{3}$ (two such groups), and $3^{1+2} \cdot D_{12}$; the first has orbit sizes $1,9^{4}, 27$, the rest have $1,9,27^{2}$.

: (iii) $r=2, q=3, d=8$ : here there are five 3 -exceptional groups; they are $2_{+}^{1+6} . X$ with $X=L_{3}(2), 2^{3} . L_{3}(2)$ or $2^{3} .7 .3$ (all with orbit sizes $1,16,112,128^{2}, 224,448$, $896,1024,1792^{2}$ ), and $2_{-}^{1+6} . Y$ with $Y=2^{4} . \mathrm{A}_{5}$ or $2^{4} . \mathrm{S}_{5}$ (all with orbit sizes 1,160 , 1280, 5120). 
7.1. Reductions. Let $G$ be a $p$-exceptional group as in the hypothesis of Theorem 7.1, and assume that $G \leq G L(V)$. We shall handle the case where $G \leq \Gamma L(V)$ and $G \not \leq G L(V)$ at the end of the proof in Section 7.4.

We begin with a technical lemma concerning the Jordan block structure of certain elements of $N_{G L(V)}(R)$. Write $J_{k}$ for a unipotent Jordan block of size $k$.

Lemma 7.2. Let $R$ be as in Table 7. Assume that the characteristic $p$ is a primitive prime divisor of $r^{2 m}-1$ or a primitive prime divisor of $r^{m}-1$ with $m$ odd, and also that $p=7$ when $(r, m)=(2,3)$. Let $t$ be an element of order $p$ in $N_{G L(V)}(R)$. Then $t$ acts on $V$ as $\left(J_{p}^{k}, J_{\ell}\right)$, where $r^{m}=k p+\ell$ and $0 \leq \ell<p$.

Proof First we consider the case where $m$ is odd and $p$ is a primitive prime divisor of $r^{m}-1$ (so $\left.\ell=1\right)$. If $R=2_{ \pm}^{1+2 m}$, then this in particular implies that $R=2_{+}^{1+2 m}$. In all cases, embedding $t R Z$ in a subgroup $G L_{m}(r)$ of $N_{G L(V)}(R) / R Z$, one can check that there exists a $t$-stable elementary abelian $r$-subgroup $A<R$ of order $r^{m}$ such that $V \downarrow$ $A$ affords the regular representation of $A$ and moreover $t$ acts fixed-point-freely on the nontrivial irreducible characters of $A$. Thus $t$ also permutes fixed-point-freely the nontrivial $A$-eigenspaces in $V$. Since $|t|=p$ and $\operatorname{dim} C_{V}(A)=1$, it follows that $t$ acts on $V$ as $\left(J_{p}^{k}, J_{1}\right)$ as stated.

Assume now that $p$ is a primitive prime divisor of $r^{2 m}-1$ (so $\ell=p-1$ ). Note that $Z(R) Z$ acts trivially on $V \otimes V^{*}, B:=R / Z(R)$ is elementary abelian of order $r^{2 m},\left(V \otimes V^{*}\right) \downarrow B$ affords the regular representation of $B$, and $t$ acts fixed-point-freely on the nontrivial irreducible characters of $B$. Thus $t$ also permutes fixed-point-freely the nontrivial $B$-eigenspaces in $V \otimes V^{*}$. As before, it follows that $t$ acts on $V \otimes V^{*}$ as $\left(J_{p}^{n}, J_{1}\right)$ with $n:=\left(r^{2 m}-1\right) / p$.

Recall [13, Theorem VIII.2.7] that the Jordan canonical form of $J_{a} \otimes J_{b}$ equals $\left(J_{a+b-1}, J_{a+b-3}, \ldots, J_{b-a+1}\right)$ if $1 \leq a \leq b<a+b \leq p$, and

$$
\left(J_{p}^{(a+b-p)}, J_{2 p-a-b-1}, J_{2 p-a-b-3}, \ldots, J_{b-a+1}\right)
$$

if $1 \leq a \leq b<p<a+b$. It follows, that $J_{a} \otimes J_{b}$ can contain no block of size between 2 and $p-1$ only when $a=b \in\{1, p-1\}$. Applying this observation to $\left(V \otimes V^{*}\right) \downarrow t$, we see that $V \downarrow t=\left(J_{p}^{c}, J_{e}^{d}\right)$ for some $c, d$ and some $e \in\{1, p-1\}$. In fact, since $\left(V \otimes V^{*}\right) \downarrow t$ contains $J_{1}$ only once, $d \leq 1$. But $p c+d e=r^{m}=k p+(p-1)$, whence $(d, e)=(1, p-1)$, as stated.

The next lemma reduces the number of possibilities for $R$ to a finite number.

Lemma 7.3. The possibilities for $R$ are as follows:

$$
\begin{aligned}
& 4 \circ 2^{1+2 m}, m \leq 10 \\
& 2_{ \pm}^{1+2 m}, m \leq 11 \\
& 3^{1+2 m}, m \leq 5 \\
& 5^{1+2 m}, m \leq 2 \\
& 7^{1+2 m}, m \leq 2 \\
& 11^{1+2}
\end{aligned}
$$

Proof First consider $R=4 \circ 2^{1+2 m}$. Assume $m \geq 11$. Here $N_{G L(V)}(R) / R Z=S p_{2 m}(2)$ and $G \leq N_{G L(V)}(R)$. Let $t \in G$ be an element of order $p$. By [22, 4.3], there are $m+3$ conjugates of $t$ which generate a subgroup of $N_{G L(V)}(R)$ covering $S p_{2 m}(2)$. In fact these conjugates generate the whole of $N_{G L(V)}(R)$ since otherwise they would generate a covering group of $S p_{2 m}(2)$; but there is no such group in dimension $2^{m}$ by [29]. It follows that $\operatorname{dim} C_{V}(t) \leq 2^{m}\left(1-\frac{1}{m+3}\right)$. Hence, as $G$ is $p$-exceptional, Lemma 2.4(ii) implies that

$$
q^{2^{m}} \leq q^{2^{m}\left(1-\frac{1}{m+3}\right)}\left|t^{G}\right| \text {. }
$$

It follows that $q^{2^{m} /(m+3)} \leq\left|t^{G}\right| \leq 2^{2 m}\left|S p_{2 m}(2)\right|$. Since $q \geq 5$ in this case (as it is $1 \bmod 4$ ), this is a contradiction for $m \geq 11$.

An entirely similar argument shows that $m \leq 11$ in the case where $R=2_{ \pm}^{1+2 m}$.

Now consider $R=r^{1+2 m}$ with $r$ odd. As above, take an element $t$ of order $p$ in $G$. By [22], there are $m+3$ conjugates of $t$ which generate a subgroup of $N_{G L(V)}(R)$ which covers 
$S p_{2 m}(r)$. Such a subgroup fixes no nonzero vectors in $V$ (note that the restriction of $V$ to a subgroup $S p_{2 m}(r)$ is the sum of two irreducible Weil modules - see [44]), and so again $\operatorname{dim} C_{V}(t) \leq r^{m}\left(1-\frac{1}{m+3}\right)$, giving

$$
q^{r^{m} /(m+3)} \leq\left|t^{G}\right| \leq r^{2 m}\left|S p_{2 m}(r)\right| .
$$

Also $r$ divides $q-1$. The bound (4) implies that $R$ is as in the conclusion, except that $R=13^{1+2}$ is also possible; but this can be ruled out by noting that only 3 conjugates of $t$ are required (rather than 4 ), by $[22,3.1]$.

Lemma 7.4. We have $r<5$.

Proof Suppose $r \geq 5$. Then $r=5,7$ or 11 by Lemma 7.3.

First consider $r=5$. Here $m \leq 2$. Suppose $m=1$, so $G / Z \leq 5^{2} . S p_{2}(5), d=\operatorname{dim} V=5$ and $q \equiv 1 \bmod 5$. As $p$ divides $|G|$ we have $p=2$ or 3 and $q=16$ or 81 . For $p=2$, Lemma 7.2 shows that an involution $t \in G$ acts on $V$ as $\left(J_{2}^{2}, J_{1}\right)$, so that $\operatorname{dim} C_{V}(t)=3$. Hence Lemma 2.4(ii) gives $16^{2} \leq\left|t^{G}\right|$. However, $\left|t^{G}\right| \leq 5^{2}$, so this is a contradiction. And for $p=3$, an element $t \in G$ of order 3 acts on $V$ as $\left(J_{3}, J_{2}\right)$ and we argue similarly.

Now suppose $m=2$ (still with $r=5$ ). Here $G / Z \leq 5^{4} \cdot S p_{4}(5), d=\operatorname{dim} V=25$ and $q \equiv 1 \bmod 5$. As $p$ divides $|G|$ we have $p=2,3$ or 13 and $q=16,81$ or $13^{4}$. Let $t \in G$ be an element of order $p$. For $p=2$, involutions in $G$ lie in a subgroup $5^{1+2} S p_{2}(5) \circ 5^{1+2} S p_{2}(5)$ acting on $V$ as a tensor product of two 5-spaces, and hence act on $V$ as either $\left(J_{2}^{2}, J_{1}\right) \otimes$ $\left(J_{2}^{2}, J_{1}\right)$ or $\left(J_{2}^{2}, J_{1}\right) \otimes I_{5}$; hence $\operatorname{dim} C_{V}(t) \leq 15$. For $p=3$, elements of order 3 in $G$ also lie in this subgroup, so act as $\left(J_{3}, J_{2}\right) \otimes\left(J_{3}, J_{2}\right)$ or $\left(J_{3}, J_{2}\right) \otimes I_{5}$; hence $\operatorname{dim} C_{V}(t) \leq 10$. And for $p=13$, elements of order 13 in $G$ act as $\left(J_{13}, J_{2}\right)$, hence $\operatorname{dim} C_{V}(t)=2$. We conclude that $\operatorname{dim} C_{V}(t) \leq 15$ in any characteristic. Now Lemma 2.4(ii) gives a contradiction.

The cases $r=7$ and $r=11$ are ruled out in entirely similar fashion.

7.2. The case $r=2$. In this section we handle the case where $r=2$. By Lemma 7.3, $R$ is either $4 \circ 2^{1+2 m}$ with $m \leq 10$, or $2_{ \pm}^{1+2 m}$ with $m \leq 11$.

Lemma 7.5. We have $m \leq 5$.

Proof Consider the case $m=6$. Here $d=\operatorname{dim} V=64, R=2_{ \pm}^{1+12}$ or $4 \circ 2^{1+12}$ and $G / R Z \leq O_{12}^{ \pm}(2)$ or $S p_{12}(2)$, respectively. Moreover $p=3,5,7,11,13,17$ or 31 .

Suppose first that $p=3$ and $R=2_{ \pm}^{1+12}$. Then $q=3$. We shall use Lemma 2.4(ii). Let $t \in G$ have order 3. Modulo $R, t$ is conjugate to an element $t_{k}$ of order 3 in a subgroup $O_{2}^{-}(2)^{k}$ of $O_{12}^{ \pm}(2)$, where $1 \leq k \leq 6$, projecting nontrivially to each factor. We can work out the Jordan form of $t_{k}$ on $V$ as an element of $2^{1+2} O_{2}^{-}(2) \otimes \cdots \otimes 2^{1+2} O_{2}^{-}(2) \otimes I_{2^{6-k}}$ : so on $V, t_{k}$ acts as $J_{2} \otimes \cdots \otimes J_{2} \otimes I_{2^{6-k}}$ where there are $k$ factors $J_{2}$. The Jordan forms of these tensor products are easily worked out, and we find that the number of Jordan blocks of $t_{k}$ is as follows:

\begin{tabular}{l|llllll}
$k$ & 1 & 2 & 3 & 4 & 5 & 6 \\
\hline $\operatorname{dim} C_{V}\left(t_{k}\right)$ & 32 & 32 & 24 & 24 & 22 & 22
\end{tabular}

By Corollary 2.10, modulo $R$ the centralizer of $t_{k}$ in $R . O_{12}^{ \pm}(2)$ is $C_{O_{12}^{-}(2)}\left(t_{k}\right)$, which contains $O_{12-2 k}(2) \times 3^{k} \cdot S_{k}$. Hence

$$
\left|t_{k}^{G}\right| \leq \frac{2^{12}\left|O_{12}^{ \pm}(2)\right|}{2^{12-2 k}\left|O_{12-2 k}(2)\right| \cdot 3^{k} \cdot k !} .
$$

For each $k$ between 1 and 6 , we check that $\left|V: C_{V}\left(t_{k}\right)\right|=3^{64-\operatorname{dim} C_{V}\left(t_{k}\right)}$ is greater than $\left|t_{k}^{G}\right|$. So this contradicts Lemma 2.4(ii).

When $p=3$ and $R=4 \circ 2^{1+12}$ we have $q=9($ as $q \equiv 1 \bmod 4$ in this case). Here an element $t \in G$ of order 3 lies in a subgroup $S p_{2}(2)^{k}$ of $S p_{12}(2)$ (modulo $R$ ) for some $k$ with $1 \leq k \leq 6$, and we argue as above.

Now suppose $p=5$. Here $q=5$ for both cases $R=4 \circ 2^{1+12}$ and $R=2_{ \pm}^{1+10}$. In the former case, an element $t \in G$ of order 5 is conjugate to an element $t_{k}$ in a subgroup $S p_{4}(2)^{k}$ of $S p_{12}(2)$, where $1 \leq k \leq 3$, projecting nontrivially to each factor. Then $t_{k}$ acts on $V$ as an 
element of a tensor product of $k$ factors $4 \circ 2^{1+4} \cdot S p_{4}(2)$ acting in dimension 4 . By Lemma 7.2 , an element of order 5 in such a factor acts as $J_{4}$, so $t_{k}$ acts on $V$ as $J_{4} \otimes \cdots \otimes J_{4} \otimes I_{64 / 4^{k}}$. Working out these tensor products of Jordan blocks, it follows that $\operatorname{dim} C_{V}\left(t_{k}\right)=16,16,13$ according as $k=1,2,3$ respectively. Moreover as above

$$
\left|t_{k}^{G}\right| \leq \frac{2^{12}\left|S p_{12}(2)\right|}{2^{12-4 k}\left|S p_{12-4 k}(2)\right| \cdot 5^{k} \cdot k !} .
$$

It now follows that $\left|V: C_{V}\left(t_{k}\right)\right|=5^{64-\operatorname{dim} C_{V}\left(t_{k}\right)}$ is greater than $\left|t_{k}^{G}\right|$ for each $k$, contradicting Lemma 2.4(ii) again. The $R=2_{ \pm}^{1+10}$ case is entirely similar.

For $p=7$, we see as above that an element $t \in G$ of order 7 is conjugate to an element $t_{k}(k=1$ or 2$)$ acting on $V$ as $\left(J_{7}, J_{1}\right) \otimes I_{8}$ or $\left(J_{7}, J_{1}\right) \otimes\left(J_{7}, J_{1}\right)$ (using Lemma 7.2 to see that the action of an element of order 7 in $4 \circ 2^{1+6} . S p_{6}(2)<S L_{8}\left(7^{a}\right)$ is $\left.\left(J_{7}, J_{1}\right)\right)$. Hence $\operatorname{dim} C_{V}\left(t_{k}\right)=16$ or 10 and we contradict Lemma 2.4 as before.

For larger values of $p$ there is only one class of elements of order $p$ in $N_{G L(V)}(R)$, and its action on $V$ can be computed as above using Lemma 7.2. We find that for $t \in G$ of order $p$, $\operatorname{dim} C_{V}(t)$ is $6,5,4$ or 4 according as $p=11,13,17$ or 31 . In each case $\left|V: C_{V}(t)\right|$ is much bigger than $\left|N_{G L(V)}(R)\right|$, contradicting Lemma 2.4 once more.

This completes the argument for $m=6$. For $m \geq 7$ the same method applies.

Lemma 7.6. $m$ is not 5 .

Proof Suppose $m=5$. The method is very similar to the previous proof, but the bounds are tighter and more work is needed for the case $q=3$.

Consider first the case where $p=3$. Let $R=2_{ \pm}^{1+10}$. Then $q=3$. If $t \in G$ is an element of order 3 , then $t$ is conjugate to an element $t_{k}$ lying in a subgroup $O_{2}^{-}(2)^{k}$ of $O_{10}^{ \pm}(2)$ and acting on $V$ as $J_{2} \otimes \cdots \otimes J_{2} \otimes I_{32 / 2^{k}}$. We compute that $\operatorname{dim} C_{V}\left(t_{k}\right)=16,16,12,12,11$ for $k=1,2,3,4,5$ respectively. If $k=1$ then Lemma 2.4 (ii) gives $3^{16}<\left|t_{1}^{G}\right|$; however $\left|t_{1}^{G}\right| \leq \mid O_{10}(2): 2^{8} .\left(3 \times O_{8}(2) \mid\right.$ which is less than $3^{16}$. Hence (again by Lemma 2.4(ii)), $G / R$ is a subgroup of $O_{10}^{ \pm}(2)$ containing no conjugates of $t_{1}$ and at least $3^{32-\operatorname{dim} C_{V}\left(t_{k}\right)} / 2^{2 k}$ conjugates of $t_{k}$ for some $k \geq 2$. However a MAGMA computation shows that there is no such subgroup.

In the case where $p=3$ and $R=4 \circ 2^{1+12}$ we have $q=9$. Here an element $t \in G$ of order 3 is conjugate to some $t_{k}$ as in the previous paragraph but no MAGMA computation is required as $9^{32-\operatorname{dim} C_{V}\left(t_{k}\right)}>\left|t_{k}^{G}\right|$ for all $k$, which contradicts Lemma 2.4.

Now consider $p=5$. Here $q=5$ and an element $t \in G$ of order 5 is conjugate to an element $t_{k}(k=1$ or 2$)$ lying in a subgroup $O_{4}^{-}(2)^{k}$ of $O_{10}(2)$ or $S p_{10}(2)$ and acting on $V$ as $J_{4} \otimes I_{8}\left(\right.$ for $k=1$ ) or $J_{4} \otimes J_{4} \otimes I_{2}$ (for $k=2$ ). So $\operatorname{dim} C_{V}\left(t_{k}\right)=8$ for $k=1,2$. However we check as above that $5^{24}>\left|t_{k}^{G}\right|$ for $k=1,2$, which contradicts Lemma 2.4(ii).

The other possible values of $p$ are $7,11,17$ and 31 . For these values there is only one class of elements of order $p$ in $N_{G L(V)}(R)$, and its action on $V$ can be computed as above using Lemma 7.2. In each case $\left|V: C_{V}(t)\right|$ is much bigger than $\left|N_{G L(V)}(R)\right|$, contradicting Lemma 2.4 once more.

Lemma 7.7. $m$ is not 4 .

Proof Suppose $m=4$. Then $p$ divides $\left|S p_{8}(2)\right|$, so is $3,5,7$ or 17 . The cases $p=7$ or 17 are easily handled as in the last paragraph of the previous proof.

Consider now the case $p=5$. Here $q=5$ and $G \leq\left(4 \circ 2^{1+8}\right) \cdot S p_{8}(2)<G L_{16}(5)$. An element $t \in G$ of order 5 is conjugate to an element $t_{k}(k=1$ or 2$)$ lying in $S p_{4}(2)^{k}$ and acting on $V$ as $J_{4} \otimes I_{4}$ or $J_{4} \otimes J_{4}$; so $\operatorname{dim} C_{V}\left(t_{k}\right)=4$ for both $k=1,2$. Hence $5^{12}<\left|t^{G}\right|$ by Lemma 2.4 (ii). It follows that $G / R$ contains no conjugates of $t_{1}$ and contains at least $5^{12} / 2^{8}$ conjugates of $t_{2}$. Using [8], one checks that the only possible maximal subgroup of $S p_{8}(2)$ containing $G / R$ is $O_{8}^{+}(2)$, and then that the only subgroup of this containing enough conjugates of $t_{2}$ are $\Omega_{8}^{+}(2)$ and $O_{8}^{+}(2)$. But these contain conjugates of $t_{1}$, a contradiction.

Now suppose $p=3$. Consider first the case where $R=4 \circ 2^{1+8}$. Here $q=9$. An element $t \in G$ of order 3 is conjugate to some $t_{k}\left(1 \leq k \leq 4\right.$ lying in $S p_{2}(2)^{k}$ and acting on $V$ as 
$J_{2} \otimes \cdots \otimes J_{2} \otimes I_{16 / 2^{k}}$, and we find that $\operatorname{dim} C_{V}\left(t_{k}\right)=8,8,6,6$ according as $k=1,2,3,4$ respectively. We compute that $9^{\operatorname{dim} V-\operatorname{dim} C_{V}\left(t_{k}\right)}>\left|t_{k}^{G}\right|$ for $k=1,3,4$. Hence $G / R$ is a subgroup of $S p_{8}(2)$ containing no conjugates of $t_{1}, t_{3}, t_{4}$, and at least $9^{8} / 2^{4}$ conjugates of $t_{2}$. One checks using [8] that there are no such subgroups.

Finally suppose $p=3$ and $R=2_{\epsilon}^{1+8}$, so $G \leq 2_{\epsilon}^{1+8} \cdot O_{8}^{\epsilon}(2)<G L_{16}(3)$. Here the usual bounding methods do not work and we use MAGMA computation along the following lines. For $\epsilon=-$, we compute that the group $R$ has a regular orbit on vectors that has 48960 images under $R . O_{8}^{-}(2)$. Moreover, $O_{8}^{-}(2)$ has 4683 subgroups of order divisible by 3 , and all of them have an orbit of length divisible by 3 in the action on 48960 points. Similarly, for $\epsilon=+$ the group $R$ has an orbit of length 128 that has 1575 images under $R . O_{8}^{+}(2)$, and $O_{8}^{+}(2)$ has 5988 subgroups with order divisible by 3; all of them have an orbit of length divisible by 3 on the set of size 1575 .

Lemma 7.8. If $m=3$, then $q=3$ and $G$ is as in conclusion (iii) of Theorem 7.1.

Proof Suppose $m=3$. Then $p$ divides $\left|S p_{6}(2)\right|$, so is 3,5 or 7 . We make heavy use of computation in this proof.

Let $p=3$. First consider $R=2_{+}^{1+6}$, so $G \leq 2_{+}^{1+6} \cdot O_{6}^{+}(2)<G L_{8}(3)$. We compute that the group $R$ has an orbit of length 16 on vectors, that has 30 images under $X:=R . O_{6}^{+}(2)$. Let $\Delta$ be this set of 30 images, and let $G=R . Y \leq X$ with $Y \leq O_{6}^{+}(2)$. Then $|Y|$ is divisible by 3. If $Y$ has an orbit $\Delta_{0}$ on $\Delta$ of length divisible by 3 , then $G$ has an orbit on vectors of length $16\left|\Delta_{0}\right|$, contrary to 3-exceptionality. We compute that the group $O_{6}^{+}(2)$ has 176 subgroups of order divisible by 3 , and all but 12 have an orbit of length divisible by 3 in the action on $\Delta$. Hence $Y$ is one of the remaining 12 groups. When pulled back to subgroups of $X$ containing $R$, all but three of these have an orbit on nonzero vectors of length divisible by 3 . The three remaining groups are 3-exceptional - they are $R . L_{3}(2), R .2^{3} . L_{3}(2)$ and $R .2^{3} .7 .3$. All three are imprimitive on $V$, preserving a decomposition into eight 1-dimensional spaces, and are as in Theorem 7.1(iii).

Now consider $R=2_{-}^{1+6}$, so $G \leq 2_{-}^{1+6} \cdot O_{6}^{-}(2)<G L_{8}(3)$. Here $R$ has an orbit on vectors of length 32 that has 45 images under $X:=R . O^{-}(6,2)$. Now $O^{-}(6,2)$ has 238 subgroups of order divisible by 3 and all but 7 have an orbit of length divisible by 3 in the action on 45 points. Pulling these remaining 7 subgroups back to subgroups of $X$ containing $R$ we see that all but two have an orbit on nonzero vectors of length divisible by 3 . The two groups are $R .\left(2^{4} . \mathrm{A}_{5}\right)$ and $R .\left(2^{4} \cdot \mathrm{S}_{5}\right)$, as in Theorem 7.1 (iii). Both are imprimitive on $V$ with a decomposition into two 4-dimensional spaces.

Finally, if $R=4 \circ 2^{1+6}$, then $q=9$ and $R$ has an orbit of length 32 that has 270 images under $X:=R . S p(6,2)$. Then $S p_{6}(2)$ has 2777 subgroups of order divisible by 3 , and all but 13 of these have an orbit of length divisible by 3 in the action on 270 points. Pulling back these 13 subgroups to subgroups of $X$ containing $R$, we find that they all have an orbit of length divisible by 3 on vectors.

Now let $p=5$, so $G \leq X:=4 \circ 2^{1+6} . S p_{6}(2)<G L_{8}(5)$. Here $R$ has an orbit on vectors of length 32 that has 135 images under $X:=R . S p_{6}(2)$. Now $S p_{6}(2)$ has 82 subgroups with order divisible by 5 and all have an orbit of length divisible by 5 in the action on 135 points.

Lastly let $p=7$. If $q=49$ the usual method using Lemma 2.4 yields a contradiction, so suppose $q=7$ and $R=2_{+}^{1+6}$ (note that 7 does not divide $\left|O_{6}^{-}(2)\right|$ so only the + type is possible). An element $t \in G$ of order 7 acts on $V$ as $\left(J_{7}, J_{1}\right)$, so Lemma 2.4(ii) implies that $G / R$ is a subgroup of $O_{6}^{+}(2)$ containing at least $7^{6} / 2^{6}$ conjugates of $t$. This implies that $G / R$ contains $\Omega_{6}^{+}(2)$. Now one computes that $R . \Omega_{6}^{+}(2)$ has an orbit on vectors of length divisible by 7 .

Lemma 7.9. If $m=2$ then $q=3$ and $G$ is a subgroup $2_{-}^{1+4} \cdot \mathrm{A}_{4}$ or $2_{-}^{1+4}$. $\mathrm{S}_{4}$ of $G L_{4}(3)$. These subgroups are imprimitive and 3-exceptional, with orbits on vectors of sizes 1, 16, 64 .

Proof Here $G$ is a subgroup of one of $2_{\epsilon}^{1+4} . O_{4}^{\epsilon}(2)<G L_{4}(3)$, or $4 \circ 2^{1+4} . S p_{4}(2)<G L_{4}(9)$ or $G L_{4}(5)$. A routine computation of all subgroups of $R . O_{4}^{\epsilon}(2)$ and $R . S p_{4}(2)$ containing $R$ gives the conclusion. 
This completes the proof of Theorem 7.1 in the case where $G \leq G L(V)$ and $r=2$.

7.3. The case $r=3$. Now suppose $r=3$. By Lemma 7.3, we have $R=3^{1+2 m}$ with $m \leq 5$. Also $G \leq R . S p_{2 m}(3)<G L(V)=G L_{3^{m}}(q)$ with $q \equiv 1 \bmod 3$.

Lemma 7.10. We have $m \leq 2$.

Proof First consider $m=5$. The bound (4) forces $q=4$ or 7 . If $q=7$ and $t \in G$ is an element of order 7, then modulo $R, t$ lies in a subgroup $S p_{6}(3) \times S p_{4}(3)$ of $S p_{10}(3)$. Hence by Lemma 7.2, $t$ acts on $V$ as $\left(J_{7}^{27}, J_{6}^{9}\right)$ and $\operatorname{dim} C_{V}(t)=36$. Now Lemma 2.4 gives a contradiction. Similarly, when $q=4$ an involution $t \in G$ lies in a subgroup $S p_{2}(3)^{k}$ and acts as a tensor product of $k$ factors $\left(J_{2}, J_{1}\right)$ with an identity matrix, whence we see that $\operatorname{dim} C_{V}(t) \leq 162$, and again Lemma 2.4 is violated.

Now let $m=4$. Then $p$ divides $\left|S p_{8}(3)\right|$. If $t \in G$ has order $p$, we see using Lemma 7.2 in the usual way that $\operatorname{dim} C_{V}(t) \leq 54$. Now Lemma 2.4 (ii) forces $p=2$ and $q=4$. The involution $t$ is conjugate to an element $t_{k}$ lying in $S p_{2}(3)^{k}(1 \leq k \leq 4)$ and acting on $V$ as a tensor product of $k$ factors $\left(J_{2}, J_{1}\right)$ with an identity matrix. Hence $\operatorname{dim} C_{V}\left(t_{k}\right)=$ $54,45,42,41$ according as $k=1,2,3,4$ respectively. We find that for each $k$ we have $\mid V$ : $C_{V}(t)|>| t_{k}^{G} \mid$, contrary to Lemma 2.4 .

The case $m=3$ is dealt with in exactly the same fashion, and we leave this to the reader.

Lemma 7.11. We have $m=1, q=4$ and $G=3^{1+2} .2$ or $3^{1+2} .6$ in $G L_{3}(4)$, as in Theorem 7.1(iii). Both are imprimitive on $V$, with orbit sizes $1,9^{4}, 27$ or $1,9,27^{2}$, respectively.

Proof Suppose $m=2$. Then $p$ divides $\left|S p_{4}(3)\right|$, so $p=2$ or 5 . The usual arguments rule out $p=5$ (for which $q=25$ ), so $p=2, q=4$ and $G \leq R . S p_{4}(3)<G L_{9}(4)$. A computation of all the subgroups of $S p_{4}(3)$ of even order shows that there are no 3-exceptional groups in this case.

Hence $m=1$. Then $p$ divides $\left|S p_{2}(3)\right|$, so $p=2, q=4$ and a computation gives the examples in the lemma.

This completes the proof of Theorem 7.1 in the case where $G \leq G L(V)$.

7.4. The semilinear case. We now complete the proof of Theorem 7.1 by handling the case where the $p$-exceptional group $G$ in the hypothesis lies in $\Gamma L(V)$ and not in $G L(V)$, where $V=V_{d}(q)$. Let $G_{0}=G \cap G L(V)$. If $p$ divides $\left|G_{0}\right|$ then $G_{0}$ is $p$-exceptional, hence is given by the linear case of the theorem which we have already proved. The only possibility is that $d=3, q=4$ and $G_{0}$ is one of the two groups in the conclusion of Lemma 7.11. Computation in $\Gamma L_{3}(4)$ reveals one further 2-exceptional group $G$ in this case, the group $3^{1+2} \cdot D_{12}$ in Theorem 7.1(iii).

Assume now that $p$ does not divide $\left|G_{0}\right|$. By Lemma 2.3, $G$ has a $p$-exceptional normal subgroup $G_{0}\langle\sigma\rangle$, where $\sigma \in \Gamma L(V) \backslash G L(V)$ is a field automorphism of order $p$. Hence $q=p^{k p}$ for some integer $k$. If $r=2$ then $q=p$ or $p^{2}$ (as $G$ is not realisable over a proper subfield of $\mathbb{F}_{q}$ ), and also $q$ is odd; so it is impossible to have $q=p^{k p}$. Therefore $r \geq 3$. Also the field automorphism $\sigma$ acts on $V$, fixing pointwise a subset $V_{d}\left(q^{1 / p}\right)$, and so $\left|V: C_{V}(\sigma)\right|=q^{\left(1-\frac{1}{p}\right) r^{m}}$. Hence Lemma 2.4(ii) gives

$$
q^{\left(1-\frac{1}{p}\right) r^{m}} \leq\left|\sigma^{G}\right| \leq r^{2 m}\left|S p_{2 m}(r)\right| .
$$

If $p \neq 2$ then $q \geq 27$ and one checks that (5) cannot hold. Hence $p=2$ and $q=4^{k}$.

If $k \geq 2$ then (5) implies that $m=1, q=16$ and $r=5$. For $k=1$, we have $r=3$ and (5) gives $m \leq 3$. Moreover if $m=3$ then $G_{0} / R$ is an odd order subgroup of $S p_{6}(3)$. Computation shows that the largest such subgroup has order $3^{7} \cdot 13$. Hence $\left|G_{0} / R\right| \leq 3^{7} \cdot 13$, and so (5) gives $2^{27} \leq 3^{14} \cdot 13$, which is false. Hence $m \leq 2$. The possibilities remaining are 
as follows, where we write $K$ for the odd order group $G_{0} / R \leq S p_{2 m}(r)$ :

$$
\begin{aligned}
& \text { (1) } G=5^{1+2} \cdot K \cdot 2<\Gamma L_{5}(16) \\
& (2) \quad G=3^{1+2} \cdot K \cdot 2<\Gamma L_{3}(4) \\
& \text { (3) } G=3^{1+4} \cdot K \cdot 2<\Gamma L_{9}(4)
\end{aligned}
$$

In cases (1) and (3) we check computationally that there are no 2-exceptional examples, and in case (2) we get the two examples $3^{1+2} \cdot S_{3}$ in the conclusion of Theorem 7.1 (iii).

This completes the proof of Theorem 7.1.

\section{8. $\mathcal{C}_{9}$ CASE I: PREliminaries And $\operatorname{Lie}(p)$}

Define $G \leq \Gamma L(V)=\Gamma L_{d}(q)\left(q=p^{a}\right)$ to be in class $\mathcal{C}_{9}$ if $G / Z$ is almost simple, with socle absolutely irreducible and not realisable over a proper subfield of $\mathbb{F}_{q}$. Let $L=\operatorname{soc}(G / Z)$, a simple group, and let $\hat{L}$ be a perfect preimage of $L$ in $G$.

The case where the simple group $L$ is of Lie type in characteristic $p$ turns out to be very easy. Define Lie $(p)$ to be the set of simple groups of Lie type in characteristic $p$, excluding $S p_{4}(2)^{\prime}, G_{2}(2)^{\prime},{ }^{2} F_{4}(2)^{\prime}$ in characteristic 2 , and ${ }^{2} G_{2}(3)^{\prime}$ in characteristic 3 . The first two of these and the last are dealt with in Sections 9 and 11 (in their guises as $A_{6}, U_{3}(3)$ and $\left.L_{2}(8)\right)$, and ${ }^{2} F_{4}(2)^{\prime}$ in the remark below after Corollary 8.2.

Lemma 8.1. Suppose that $G \leq \Gamma L(V)=\Gamma L_{d}(q)\left(q=p^{a}\right)$ is in class $\mathcal{C}_{9}$ and $L=$ $\operatorname{soc}(G / Z) \in \operatorname{Lie}(p)$. If $G$ is p-exceptional, then $G$ (and $\hat{L}$ ) is transitive on $P_{1}(V)$.

Proof From the structure of the exceptional Schur multipliers of simple groups in Lie $(p)$ (see [27, Table 5.1.D] for example), we see that the perfect group $\hat{L}$ must be an image of the simply connected group of type $L$ (since the extra parts of such multipliers are always $p$-groups which hence act trivially on irreducible modules in characteristic $p$ ). Therefore it follows from $[9$, Theorem 4.3(c)] that a Sylow $p$-subgroup $P$ of $\hat{L}$ fixes a unique 1-space in $V$. If $G$ is $p$-exceptional then so is $\hat{L}$, and so every nonzero vector in $V$ is fixed by an $\hat{L}$-conjugate of $P$. For 1 -spaces $\langle v\rangle,\langle w\rangle$ fixed by $P^{g}, P^{h}$, we then have $\langle v\rangle g^{-1} h=\langle w\rangle$, and the conclusion follows.

Corollary 8.2. If $G$ is as in Lemma 8.1 and is p-exceptional, then $G$ is transitive on $V^{\sharp}$.

Proof By Lemma 8.1, $\hat{L}$ is transitive on $P_{1}(V)$. Hence $\hat{L}$ is given by Hering's Theorem (see [30, Appendix 1]): so $\hat{L}$ is $S L_{d}(q), S p_{d}(q)$ or $G_{2}(q)$ ( $q$ even, $d=6$ ), and in each case $\hat{L}$ is transitive on $V^{\sharp}$.

Remark The case where $L=\operatorname{soc}(G / Z)={ }^{2} F_{4}(2)^{\prime}$ and $p=2$ is quickly ruled out, as follows. Suppose $G$ is 2-exceptional. The 2-modular characters of $L$ are given in [26], and the bound in Lemma 2.4(i) implies that $d=26, q=2$. As 13 does not divide $\left|V^{\sharp}\right|=2^{26}-1$, an element $t \in L$ of order 13 fixes a nonzero vector $v$. By 2-exceptionality, $v$ is also fixed by a Sylow 2-subgroup $P$ of $L$. But $t$ and $P$ generate $L$ (see $[8$, p.74]), so this is impossible.

\section{9. $\mathcal{C}_{9}$ Case II: Alternating groups}

In this section we deal with the case where the simple group $L=\operatorname{soc}(G / Z)$ is an alternating group. We prove

Theorem 9.1. Let $G \leq \Gamma L(V)=\Gamma L_{d}(q)\left(q=p^{a}\right)$ be such that $G / Z$ is almost simple with socle $L \cong \mathrm{A}_{c}$, an alternating group with $c \geq 5$, and suppose the perfect preimage $\hat{L}$ of $L$ in $G$ is absolutely irreducible on $V$ and realisable over no proper subfield of $\mathbb{F}_{q}$. Assume $G$ is p-exceptional and not transitive on $V^{\sharp}$. Then one of the following holds:

(i) $G=\mathrm{A}_{c}$ or $\mathrm{S}_{c}$ with $c=2^{r}-2$ or $2^{r}-1$, with $V$ the deleted permutation module for $G$ over $\mathbb{F}_{2}$, of dimension $d=c-2$ or $c-1$ respectively;

(ii) $\hat{L}=S L_{2}(5) \triangleleft G<\Gamma L_{2}(9)$, with orbit sizes $1,40,40$ on vectors.

Conversely, the groups $G$ in (i) are p-exceptional. 
We shall need the following.

Proposition 9.2. If $r$ is a prime with $r \leq c$ (and $c \geq 5$ ), then $\mathrm{A}_{c}$ is generated by two of its Sylow r-subgroups.

Proof If $r=2$ this follows from [21], so assume that $r>2$. Write $c=k r+s$ for integers $k, s$ with $0 \leq s \leq r-1$.

First consider the case where $k=1$. If $s=0$, observe that $(12 \cdots r)$ and $(12 \cdots r)(123)$ are both $r$-cycles, and the group they generate contains a 3-cycle, hence is $\mathrm{A}_{r}$. If $s=1$ then $c=r+1$ and the $r$-cycles $(12 \cdots r),(23 \cdots r+1)$ generate $\mathrm{A}_{c}$ as their commutator contains a 3-cycle. A similar argument applies for $s=2$, taking $(12 \cdots r)$ and $(34 \cdots r+2)$ : the group these generate is a primitive group containing both an $r$-cycle and a 5 -cycle, hence is $\mathrm{A}_{c}$. Finally, if $s>2$ then $(12 \cdots r)$ and $(s+1 s+2 \cdots c)$ generate a primitive group containing an $r$-cycle fixing more than 2 points, hence generate $\mathrm{A}_{c}$ by Jordan's Theorem $[45,13.9]$.

Now suppose $k \geq 2$. Take $R$ to be a Sylow $r$-subgroup of $\mathrm{A}_{c}$ containing the $r$-cycles $(1 \cdots r),(2 r-1 \cdots 3 r-2), \ldots,(2 i r-2 i+1 \cdots(2 i+1) r-2 i)$ up to the maximal $i$ such that $(2 i+1) r-2 i \leq c$, and take $S$ to be a Sylow $r$-subgroup containing the $r$-cycles $(r \cdots 2 r-1)$, $(3 r-2 \cdots 4 r-3), \ldots,((2 i-1) r-2 i+2 \cdots 2 i r-2 i+1)$. Then the group generated by $R$ and $S$ contains a subgroup $\mathrm{A}_{c-r+1}$, and now we can include a further $r$-cycle in $S$ to generate $\mathrm{A}_{c}$.

Now we embark upon the proof of Theorem 9.1. Let $G \leq \Gamma L(V)=\Gamma L_{d}(q)\left(q=p^{a}\right)$ be as in the hypothesis, with $L=\operatorname{soc}(G / Z) \cong \mathrm{A}_{c}$. Note that $|\operatorname{Out}(L)|=2$ or 4 , so $p$ divides $G \cap G L(V)$ which is therefore $p$-exceptional by Lemma 2.1. Hence we may replace $G$ by $G \cap G L(V)$ and assume that $G \leq G L(V)$. By Lemma 2.4, together with Lemma 9.2, we have

$$
d=\operatorname{dim} V \leq 2 \log _{q}(c !)-2 \log _{q}\left(\left(\frac{c !}{2}\right)_{p}\right),
$$

where $n_{p}$ denotes the $p$-part of an integer $n$.

Since $A_{5} \cong L_{2}(5) \cong L_{2}(4), \mathrm{A}_{6} \cong L_{2}(9)$ and $\mathrm{A}_{8} \cong L_{4}(2)$, Lemma 8.1 shows that we may exclude these groups from consideration in these characteristics.

Lemma 9.3. One of the following holds:

(i) $\hat{L}=L$ and $V$ is the deleted permutation module over $\mathbb{F}_{p}$;

(ii) $c \leq 13$ and $d \leq 32$.

Proof Suppose $V$ is not the deleted permutation module. Assume that $d>250$. Then the bound (6) forces $c>40$. By [25, Theorem 7] (for $\hat{L}=\mathrm{A}_{c}$ ) and [43] (for $\hat{L}=2 . \mathrm{A}_{c}$ ), we have $d \geq \frac{1}{4} c(c-5)$. But this is greater than the upper bound for $d$ given by (6) when $c>40$. Hence $d \leq 250$. Now all the possibilities for the $\mathbb{F}_{q} \hat{L}$-module $V$ are given by [24]. We check that the only possibilities for which the bound (6) is satisfied have $c \leq 13$ and $d \leq 32$.

Lemma 9.4. Suppose $V$ is the deleted permutation module for $\hat{L}=\mathrm{A}_{c}$ over $\mathbb{F}_{p}$. Then $p=2$, $c=2^{r}-1$ or $2^{r}-2$, and $V$ has dimension $d=c-1$ or $c-2$ respectively. Moroever, in these representations $\mathrm{A}_{c}$ and $\mathrm{S}_{c}$ are 2-exceptional.

Proof Here $V=S /(S \cap T)$ where $S=\left\{\left(a_{1}, \ldots, a_{c}\right) \in \mathbb{F}_{p}^{c}: \sum a_{i}=0\right\}, T=\{(a, \ldots, a)$ : $\left.a \in \mathbb{F}_{p}\right\}$. If $p \geq 5$ define

$$
v=\left(1,2,-3,1,-1,2,-2, \ldots, \frac{p-3}{2},-\frac{p-3}{2}, 0, \ldots, 0\right) \in S
$$

and let $x=v+(S \cap T) \in V$. Then $(\hat{L})_{x}=\mathrm{A}_{c-p} \times H$ where $H \leq \mathrm{A}_{p}$ and $|H|$ is coprime to $p$; hence $p$ divides $\left|x^{\hat{L}}\right|$, contradicting the fact that $G$ is $p$-exceptional. If $p=3$ and $c \neq 6$ the same argument applies, taking $v=(1,1,-1,-1,0, \ldots, 0)$; and if $p=3$ and $c=6$ then taking the same $v$, we have $\left|\hat{L}_{x}\right|_{3}=3$, so $\left|x^{\hat{L}}\right|$ is divisible by $|\hat{L}|_{3} / 3=3$, again a contradiction. 
Hence $p=2$. Here $d=\operatorname{dim} V=c-(c, 2)$. For $c$ odd, the orbit sizes of $\hat{L}=\mathrm{A}_{c}$ on nonzero vectors are $\left(\begin{array}{l}c \\ i\end{array}\right)$ for $1 \leq i \leq c-1$; and for $c$ even the orbit sizes are $\left(\begin{array}{c}c \\ 2 i\end{array}\right)(i \neq c / 2)$ and also $\frac{1}{2}\left(\begin{array}{c}c \\ c / 2\end{array}\right)$ when $4 \mid c$. It follows that we get 2-exceptional examples when $c=2^{r}-1$ or $2^{r}-2$. And when $c$ is not of one of these forms, let $2^{e}$ be the smallest power of 2 that is missing in the binary expansion of $c$; then $\left(\begin{array}{c}c \\ 2^{e}\end{array}\right)$ is even, so $\hat{L}$ (and hence $G$ ) is not 2-exceptional, a contradiction.

Assume from now on that $V$ is not the deleted permutation module.

Lemma 9.5. We have $c \leq 7$.

Proof From [24] and (6), we see that the possibilities for $c, d, p$ and $q$ are as in the following table:

\begin{tabular}{|l|l|l|l|l|}
\hline Case & $c$ & $d$ & $p$ & $q$ \\
\hline 1 & 13 & 32 & 3 & 3 \\
2 & 12 & 16 & 2,3 & 4,3 \\
3 & 11 & 16 & $2,3,5,7$ & $p$ or $p^{2}$ \\
4 & 10 & 8 & 5 & 5 \\
5 & & 16 & 2,3 & 2,3 \\
6 & 9 & 8 & $2,3,5,7$ & $p$ \\
7 & & 21 & 3 & 3 \\
8 & 8 & 8 & $3,5,7$ & $p$ \\
9 & & 13 & 3 & 3 \\
\hline
\end{tabular}

In cases 1, 2, 3, 4 and 7 we adopt a unified approach: for the respective primes $r=13,11,11,7$ and 7 we observe that $r$ does not divide $q^{d}-1$, and hence there is a nonzero vector $v \in V$ fixed by an element $t \in \hat{L}$ of order $r$. As $G$ is $p$-exceptional, $v$ is also fixed by a Sylow $p$-subgroup $P$ of $\hat{L}$. However it is easy to see that $\langle t, P\rangle=\hat{L}$ in all these cases, so this is a contradiction.

Now consider case 5 . Here $c=10, p=2$ or 3 and $d=\operatorname{dim} V=16$. The Brauer character $\chi$ of $V$ is given in [26]. For $p=3$ we have $\chi(t)=1$ where $t$ is a $5 B$-element of $\hat{L}=2 . \mathrm{A}_{10}$; this implies that $t$ fixes a nonzero vector $v$-however $\langle t, P\rangle=\hat{L}$ for any Sylow 3-subgroup $P$, so this is not possible. For $p=2$ we apply the same argument with $t$ a $9 A$-element of $\hat{L}=\mathrm{A}_{10}$, noting that $t$ and any Sylow 2-subgroup generate $\hat{L}$.

Next consider case 6 . For $p=2$ we have $\hat{L}=\mathrm{A}_{9}<O_{8}^{+}(2)=O(V)$, and $\hat{L}$ has two orbits on nonzero vectors (see [8]), one of which has even size, contrary to 2-exceptionality. For $p=3$ or 5 we argue as above that an element $t$ in class $7 A$ or $9 A$ fixes a nonzero vector, and generates $\hat{L}$ with any Sylow $p$-subgroup, a contradiction. The case $p=7$ requires a little more argument. Let $S$ be a Sylow 3 -subgroup of $\hat{L}=2$. $A_{9}$. Since $|V|=7^{8} \equiv 4 \bmod 9$, there is an orbit of $S$ on nonzero vectors of size 1 or 3 , and hence there is a nonzero vector $v$ which is fixed by a subgroup $S_{0}$ of $S$ of index 3. However, $S_{0}$ and any Sylow 7-subgroup generate $\hat{L}$, so this is impossible.

Now consider case 8 . For $p=5$ we argue with the Brauer character that an element in class $4 A$ in $\hat{L}=2 . A_{8}$ fixes a nonzero vector; but this element generates $\hat{L}$ with any Sylow 5 -subgroup. For $p=3$, the Brauer character $\chi$ of $V$ shows that an element $t \in \hat{L}$ of order 7 fixes a nonzero vector $v$. Then $v$ is stabilised by $\langle t, P\rangle$ for some Sylow 3-subgroup $P$ of $\hat{L}$, and this contains 2. $A_{7}$. However $\chi \downarrow 2 . A_{7}$ is a sum of two irreducibles of degree 4 , so $2 . A_{7}$ does not fix a nonzero vector, a contradiction. For $p=7$ we argue similarly using a $4 B$-element $t$.

Finally, consider case 9. Here a $7 A$ element $t \in \hat{L}=\mathrm{A}_{8}$ fixes a nonzero vector $v$, and so $v$ is fixed by $\langle t, P\rangle$ for some Sylow 3 -subgroup $P$ of $\hat{L}$. This contains $A_{7}$. However we see from [26] that $\mathrm{A}_{7}$ acts irreducibly on $V$, so this is impossible.

Lemma 9.6. $c$ is not 7 . 
Proof Suppose $c=7$. Here [24] and (6) show that $d, q$ are as in the following table of possibilities:

\begin{tabular}{|l|l|l|}
\hline Case & $d$ & $q$ \\
\hline 1 & 15 & 3 \\
2 & 13 & 3,5 \\
3 & 9 & 7 \\
4 & 8 & 5 \\
5 & 6 & $4,9,7$ \\
6 & 4 & $2,9,25,7$ \\
7 & 3 & 25 \\
\hline
\end{tabular}

In cases $1-4$, an element $t$ of order 5 or 7 fixes a nonzero vector, and generates $\hat{L}$ with any Sylow $p$-subgroup, giving a contradiction as usual.

Consider case 5. For $p=7$, we argue as before with an element of order 5. For $p=3$, the Brauer character shows that an element $t$ of order 5 in $\hat{L}=2 . \mathrm{A}_{7}$ fixes a nonzero vector $v$, and so $v$ is fixed by $\langle t, P\rangle$ for some Sylow 3 -subgroup $P$ of $\hat{L}$. This contains 2 . $\mathrm{A}_{6}$, which is irreducible on $V$, a contradiction. Finally for $p=2$, we have $\hat{L}=3 . \mathrm{A}_{7}<3 . M_{22}<S U_{6}(2)<$ $S L(V)$ (see [8, p.39]). From [8] we see that $M_{22}$ is transitive on the set of 672 non-isotropic 1-spaces in $V$, and $\mathrm{A}_{7}$ has 3 orbits on these, so that one orbit must have even size, contrary to $p$-exceptionality.

Now consider case 6 . For $p=3$ or 5 , we argue as usual with an element of order 7 which fixes a vector. For $p=2$ we have $\hat{L}=\mathrm{A}_{7}<S L_{4}(2)=S L(V)$ and $\hat{L}$ is transitive on $V^{\sharp}$, contrary to assumption. And for $p=7$ we have $\hat{L}=2 . \mathrm{A}_{7}<S L_{4}(7)$; by [30, Appendix 2], $\hat{L}$ has two orbits on nonzero vectors, one of which has size divisible by 7 .

Finally, in case 7 the Brauer character shows that there is an element $t$ of order 3 fixing a nonzero vector $v$, so $v$ is fixed by $\langle t, P\rangle$ for some Sylow 5 -subgroup $P$, and this contains $\mathrm{A}_{5}$; but a subgroup $\mathrm{A}_{5}$ must be irreducible on $V$, a contradiction.

Lemma 9.7. We have $c=5, d=2, q=9$ and $\hat{L}=S L_{2}(5)<S L_{2}(9)=S L(V)$, with orbit sizes 40,40 on nonzero vectors.

Proof We know that $c=5$ or 6 . Recall that $p=3$ when $c=5$, and $p \neq 3$ when $c=6$. Hence [24] and (6) show that $d, q$ are as in the following table of possibilities:

\begin{tabular}{|l|l|l|l|}
\hline Case & $c$ & $d$ & $q$ \\
\hline 1 & 6 & 4 & 2,5 \\
2 & 6 & 3 & 4,25 \\
3 & 5 & 6 & 3 \\
4 & 5 & 3 & 9 \\
5 & 5 & 2 & 9 \\
\hline
\end{tabular}

Consider case 1. If $p=2$ then $\hat{L}<S L_{4}(2)$ is transitive on nonzero vectors. And if $p=5$, the Brauer character shows that there is an element $t$ of order 3 fixing a nonzero vector $v$, so the stabiliser of $v$ contains $\langle t, P\rangle$ for some Sylow 5 -subgroup $P$ of $\hat{L}=2 . \mathrm{A}_{6}$. So this stabiliser contains 2. $\mathrm{A}_{5}$; but a subgroup 2. $\mathrm{A}_{5}$ is irreducible on $V$, a contradiction. The same argument deals with case 2 for $p=5$; and in case 2 for $p=2$ we have $\hat{L}=3 . \mathrm{A}_{6}<S L_{3}(4)=S L(V)$, and [30] shows that $\hat{L}$ has 2 orbits on nonzero vectors, one of which has even size.

In cases 3 and 4 we observe that an element of order 5 in $\hat{L}$ fixes a vector and generates $\hat{L}$ with any Sylow 3 -subgroup. Finally, case 5 gives the example in the conclusion.

This completes the proof of Theorem 9.1.

\section{0. $\mathcal{C}_{9}$ CASE III: Sporadic groups}

In this section we deal with the case where the simple group $L=\operatorname{soc}(G / Z)$ is a sporadic group. We prove 
Theorem 10.1. Let $G \leq \Gamma L(V)=\Gamma L_{d}(q)\left(q=p^{a}\right)$ be such that $G / Z$ is almost simple with socle $L$ a sporadic group, and suppose the perfect preimage $\hat{L}$ of $L$ in $G$ is absolutely irreducible on $V$ and realisable over no proper subfield of $\mathbb{F}_{q}$. Assume $G$ is p-exceptional on $V$. Then one of the following holds:

(i) $M_{11} \triangleleft G<G L_{5}(3)$, orbit sizes $1,22,220$;

(ii) $M_{23}=G<G L_{11}(2)$, orbit sizes $1,23,253,1771$.

We shall need the following result, analogous to Proposition 9.2.

Proposition 10.2. Let $T$ be a sporadic simple group and $p$ a prime dividing $T$. Then there exists Sylow p-subgroups $S_{1}, S_{2}$ such that $T=\left\langle S_{1}, S_{2}\right\rangle$.

Proof For all sporadic simple groups except for $T h, J_{4}, L y, B$ and $M$, permutation representations exist in MAGMA [4] (for the larger ones using the generators given in the online Atlas [46]). This allows a Sylow $p$-subgroup to be constructed and a conjugate that generates $T$ can then be found.

For the remaining five sporadics we use three strategies. Let $H$ be a maximal subgroup of $T$ such that $p$ divides $|T: H|$ and suppose that $H$ has Sylow $p$-subgroups $S_{1}$ and $S_{2}$ such that $\left\langle S_{1}, S_{2}\right\rangle=H$ or a normal subgroup of index coprime to $p$. Then for each $i \in\{1,2\}$ we can find a Sylow $p$-subgroup $S_{i}^{\prime}$ of $T$ properly containing $S_{i}$ and since $H$ is maximal in $T$ it follows that $\left\langle S_{1}^{\prime}, S_{2}^{\prime}\right\rangle=T$. Here we list the sporadic groups, primes $p$ and maximal subgroups $H$ for which we used this method:

\begin{tabular}{|l|l|l|}
\hline T & $p$ & $H$ \\
\hline Th & $3,5,7$ & $2^{5} \cdot L_{5}(2)$ \\
& 2 & $\mathrm{~S}_{5}$ \\
$J_{4}$ & $2,3,11$ & $L_{2}(32): 5$ \\
$L y$ & 2,3 & $5^{3} \cdot L_{3}(5)$ \\
& 5 & $2 \mathrm{~A}_{11}$ \\
$B$ & $2,3,5$ & $T h$ \\
& 7 & $F i_{23}$ \\
$M$ & $2,3,5,7,11,13$ & $2 B$ \\
\hline
\end{tabular}

Next, if the only maximal subgroup of $T$ containing a Sylow $p$-subgroup $S$ normalises $S$ then $T$ will be generated by any pair of Sylow $p$-subgroups. This is true for $(T, p)=\left(J_{4}, 43\right)$, $(L y, 67)$, and $(B, 47)$. Finally, if the order of a Sylow $p$-subgroup of $T$ is $p$ and there are two elements of order $p$ whose product has order a prime $r$ such that there are no maximal subgroups of $T$ with order divisible by $p r$ then $T$ is generated by these two elements of order $p$. The existence of such primes can be checked by either doing random searches using the matrix representations available in the online Atlas [46] or by doing character table calculations in GAP [15]. This method was used for the groups and primes listed below.

\begin{tabular}{|l|l|l|}
\hline$T$ & $p$ & $r$ \\
\hline Th & 13,19 & 31 \\
& 31 & 19 \\
$J_{4}$ & $5,23,29,31,37$ & 43 \\
& 7 & 37 \\
Ly & $7,31,37$ & 67 \\
& 11 & 37 \\
$B$ & $11,13,17,19,31$ & 47 \\
& 23 & 31 \\
$M$ & $17,19,23,29,31,41,47,59$ & 71 \\
& 71 & 59 \\
\hline
\end{tabular}

Now we embark upon the proof of Theorem 10.1. Let $G \leq \Gamma L(V)=\Gamma L_{d}(q)\left(q=p^{a}\right)$ be as in the hypothesis, with $L=\operatorname{soc}(G / Z)$ a sporadic group. Note that $|\operatorname{Out}(L)| \leq 2$, so 
as in the previous section we may assume that $G \leq G L(V)$. By Lemma 2.4, together with Proposition 10.2, we have

$$
d=\operatorname{dim} V \leq 2 \log _{q}\left|G: N_{G}(P)\right|,
$$

where $P \in \operatorname{Syl}_{p}(G)$.

Lemma 10.3. $L$ is not $M, B M, F i_{24}, F i_{23}, T h, L y, H N$ or $O^{\prime} N$.

Proof Suppose $L$ is one of these groups other than $M$. Then (7) implies that $d<250$, whence $L$ and $V$ are in the list in [24]. We check that for all possibilities, (7) fails. And if $L=M$ then (7) gives $d<360$, whereas any nontrivial representation of $M$ has dimension greater than this (see $[27,5.3 .8]$ ).

Lemma 10.4. $L$ is not $J_{4}, \mathrm{Fi}_{22}, \mathrm{Co}_{1}, \mathrm{Co}_{2}, \mathrm{Co}_{3}, \mathrm{Suz}, \mathrm{Ru}$ or $\mathrm{He}$.

Proof Suppose $L$ is one of these groups. Then $d<250$ by (7), so [24] together with (7) imply that $L, d, p$ are as in the following table:

\begin{tabular}{|l|l|l|}
\hline$L$ & $d$ & $p$ \\
\hline$J_{4}$ & 112 & 2 \\
$F i_{22}$ & 27,78 & 2 \\
$C o_{1}$ & 24 & any \\
$C o_{2}$ & 22 & 2 \\
& 23 & $p>2$ \\
$C o_{3}$ & 22 & 2,3 \\
& 23 & $p>3$ \\
$S u z$ & 12 & any \\
$R u$ & 28 & 2 \\
$H e$ & 51 & 2 \\
\hline
\end{tabular}

We deal with each of these in turn.

Let $L=J_{4}$. From [8, p.190] we see that there is a maximal subgroup $H=2^{10} . S L_{5}(2)$ fixing a nonzero vector $v \in V$. Hence $v$ is stabilised by the subgroup generated by $H$ and a Sylow 2-subgroup of $L$, which is $L$, a contradiction.

Now let $L=F i_{22}$. Here $(d, q)=(27,4)$ or $(78,2)$. Since $q^{d}-1$ is not divisible by 13 , there is an element $t$ of order 13 fixing a vector, and $L$ is generated by $t$ together with any Sylow 2-subgroup.

Next consider $L=C o_{1}$. A subgroup $H=\mathrm{Co}_{2}$ of $L$ fixes a nonzero vector $v$, and together with any Sylow $p$-subgroup generates $L$, provided $p \neq 11$ or 23 . For $p=11,23$ consider $V \downarrow M_{24}$. Using [26] we see that this is $V_{1} \oplus V_{23}$, where $V_{23}$ is the deleted permutation module for $M_{24}$. Hence for an element $t \in L$ of order $p$ we have $\operatorname{dim} C_{V}(t)=4$ or 2 according as $p=11$ or 23. Now Lemma 2.4(ii) gives a contradiction.

Now let $L=\mathrm{Co}_{2}$. If $p=2$ we see from [8, p.154] that there is a vector stabilised by $U_{6}(2) .2$, and this generates $L$ with any Sylow 2 -subgroup. If $p=3$ or 5 then there is a vector stabilised by an element of order 23, and this generates $L$ with any Sylow 2 -subgroup. Finally, for $p \geq 7$, consideration of $V \downarrow M_{23}$ shows that $\operatorname{dim} C_{V}(t) \leq 5$ for an element $t$ of order $p$, and now Lemma 2.4 gives a contradiction.

Now consider $L=\mathrm{Co}_{3}$. For $p=2$, there is a cyclic subgroup $H=C_{11}$ fixing a vector; and for $p=3,5$ subgroups $H=M c L, M_{23}$, respectively, fix a vector. Now observe that in each case $H$ generates $L$ with any Sylow $p$-subgroup. And for $p \geq 7$ we argue just as for $\mathrm{Co}_{2}$.

Next let $L=S u z$. For $p \neq 3,11$, there is an element of order 11 fixing a vector, and this generates $L$ with any Sylow $p$-subgroup. For $p=3$ there is a subgroup $U_{5}(2)$ fixing a vector and generating $L$ with any Sylow 3-subgroup. Finally, if $p=11$ then $q=121$ and (7) fails.

Now let $L=R u$. Here a 13-element fixes a vector and generates $L$ with any Sylow 2-subgroup. 
Finally, for $L=H e$, a 17-element fixes a vector and generates $L$ with any Sylow 2subgroup.

Lemma 10.5. $L$ is not $M c L, H S, J_{1}, J_{2}$ or $J_{3}$.

Proof Suppose $L$ is one of these groups. Then [24] together with (7) imply that $L, d, p$ are as in the following table:

\begin{tabular}{|l|l|l|}
\hline$L$ & $d$ & $p$ \\
\hline$M c L$ & 21 & 3,5 \\
& 22 & 2,7 \\
$H S$ & 20 & 2 \\
& 21 & 5 \\
& 22 & 3 \\
$J_{3}$ & 9 & 2 \\
& 18 & 2 \\
$J_{2}$ & 6 & any \\
& 14 & any \\
& 36 & 2 \\
$J_{1}$ & 7 & 11 \\
& 20 & 2 \\
\hline
\end{tabular}

Let $L=M c L$. For each $p$ we produce a subgroup $H$ of $L$ stabilizing a nonzero vector: for $p=2,3$, take $H=5^{1+2}$ (as 5 is coprime to $\left|V^{\sharp}\right|$ ); for $p=5$ take $H=C_{11}$; and for $p=7$ take $H=M_{11}$, noting that from [26] the Brauer character of $L$ on $V$ restricts to $M_{11}$ as $1+\chi_{10}+\chi_{11}$. From [8, p.100], we check that each of these subgroups $H$ generates $L$ together with any Sylow $p$-subgroup, which is a contradiction.

Now consider $L=H S$. For $p=2$, we see from [26] that the value of the Brauer character of $L$ on elements in classes $5 B, 5 C$ is 0 , and hence such elements fix a nonzero vector in $V$. By [8, p.80], $L$ has only two classes of maximal subgroups of odd index, and between them they meet only one class of elements of order 5 . This is a contradiction. For $p=3$ observe that the restriction of $V$ to a subgroup $M_{22}$ is $V_{1}+V_{21}$ where $V_{21}$ is the deleted permutation module. Now $M_{22}$ contains a Sylow 3-subgroup of $L$, and if $t \in M_{22}$ is an element of order 3 acting on 22 points with cycle-type $\left(3^{6}, 1^{4}\right)$, then $\operatorname{dim} C_{V}(t)=10$. This leads to a contradiction via Lemma 2.4(ii). Finally for $p=5, V$ restricts to $M_{22}$ as the deleted permutation module, and hence $M_{21}=L_{3}(4)$ fixes a vector; but $L_{3}(4)$ generates $L$ with any Sylow 5-subgroup.

Next let $L=J_{3}$. Here an element of order 17 fixes a vector, and generates $L$ with any Sylow 2-subgroup.

Now let $L=J_{2}$. For $d=14$ or 36 we produce a subgroup $H$ of $L$ fixing a vector and generating $L$ with any Sylow $p$-subgroup, as follows: if $p=2$ or $5, H=C_{7}$ (if $p=2$ use the Brauer character value); if $p=3, H=5^{2}$; and if $p=7, H=C_{5}$ generated by a $5 A$-element (which fixes a vector by consideration of its Brauer character value). For $d=6$ we have $L<P S p_{6}(q)$ for $q=4,5,9$ or 49 . In the first three cases the orbit sizes of $\hat{L}$ on vectors can be found in [30] $(q=4,5)$ and [3, Table 5] $(q=9)$, and in each case there is a size divisible by $p$, a contradiction. And for $q=49$ a $5 A$-element fixes a vector and generates $L$ with any Sylow 7-subgroup.

Finally let $L=J_{1}$. Here an element of order 19 fixes a vector in both modules, and generates $L$ with any Sylow $p$-subgroup.

Lemma 10.6. If $L$ is a Mathieu group then one of the following holds:

(i) $M_{11} \triangleleft G<G L_{5}(3)$, orbit sizes $1,22,220$;

(ii) $M_{23}=G<G L_{11}(2)$, orbit sizes $1,23,253,1771$. 
Proof Here (7) and [24] imply that the possibilities for $L, p, d$ are as follows:

\begin{tabular}{|l|l|l|}
\hline$L$ & $d$ & $p$ \\
\hline$M_{24}$ & 11,44 & 2 \\
& 22 & 3 \\
& 23 & 5 \\
$M_{23}$ & 11,44 & 2 \\
& 22 & 3 \\
$M_{22}$ & 10 & any \\
& $6,15,34$ & 2 \\
& 21 & 3 \\
$M_{12}$ & 10 & 2 \\
& $6,10,15$ & 3 \\
& 11 & 5 \\
$M_{11}$ & 10 & 2 \\
& 5,10 & 3 \\
\hline
\end{tabular}

Let $L=M_{24}$. For $p=2$, an element of order 11 fixes a vector and generates $L$ with any Sylow 2-subgroup. And for $p=3$ or $5, V$ is the deleted permutation module and the orbits of the vectors $\left(1,1,-1,-1,0^{20}\right)(p=3)$ and $\left(1,1,2,2,-1,0^{19}\right)(p=5)$ have size divisible by $p$.

Now consider $L=M_{23}$. For $p=2$ and $d=11$ there are two possible modules. In both cases the orbit sizes are given in [3, p.170], and one of them gives the 2-exceptional example in conclusion (ii) of the lemma. For $d=44$ we argue as follows. The group $L$ has one class of involutions, and can be generated by three of them; hence $\operatorname{dim} C_{V}(t) \leq \frac{2}{3} \operatorname{dim} V<30$. Now Lemma 2.4(ii) gives a contradiction. Finally, for $p=3, V$ is the deleted permutation module and the orbit of the vector $\left(1,1,-1,-1,0^{19}\right)$ has size divisible by 3 .

Next let $L=M_{22}$. Consider $p=2$. For $d=10,15$ or 34 , an element of order 7 fixes a vector and generates $L$ with any Sylow 2-subgroup. And for $d=6$ we have $\hat{L}=3 . M_{22}<$ $S U_{6}(2)$ and from [8, p.39] we see that $\hat{L}$ is transitive on the 672 non-isotropic vectors in $V$, contrary to 2-exceptionality. For $p=3$ and $d=21, V$ is the deleted permutation module, dealt with in the usual way. It remains to consider $d=10$ in characteristics $3,5,7$ and 11 . For $p=11$, an element of order 7 fixes a vector and generates $L$ with any Sylow $p$-subgroup. And for $p=3,5,7$ we have $\hat{L}=2 . M_{22}<S L_{10}(q)$ with $q=9,25,7$ respectively, and a Magma computation reveals the existence of orbits of size divisible by $p$.

Now let $L=M_{12}$. For $p=2$ or $5, V$ is the deleted permutation module, dealt with in the usual way. Next let $p=3$. For $d=10$ or 15 , an element of order 5 fixes a vector and generates $L$ with any Sylow 3 -subgroup. And for $d=6$ we have $\hat{L}=2 . M_{12}<S L_{6}(3)$ and $[3$, p.170] shows that there is an orbit size divisible by 3 .

Finally, let $L=M_{11}$. For $p=2, V$ is the deleted permutation module. For $p=3$ and $d=5$ the orbit sizes are given by [30], giving the example in part (i) of the lemma. And for $p=3, d=10$ there are three possible modules $V$, and a MAGMA computation shows that for each of these there is an orbit size divisible by 3 .

This completes the proof of Theorem 10.1.

\section{1. $\mathcal{C}_{9}$ CASE IV: $\operatorname{Lie}\left(p^{\prime}\right)$}

In this section we deal with the case where the simple group $L=\operatorname{soc}(G / Z)$ is a simple group of Lie type in $p^{\prime}$-characteristic. We prove

Theorem 11.1. Let $G \leq \Gamma L(V)=\Gamma L_{d}(q)\left(q=p^{a}\right)$ be such that $G / Z$ is almost simple with socle $L$ a simple group of Lie type in $p^{\prime}$-characteristic, and $L$ is not isomorphic to an alternating group. Suppose the perfect preimage $\hat{L}$ of $L$ in $G$ is absolutely irreducible on $V$ and realisable over no proper subfield of $\mathbb{F}_{q}$. Assume $G$ is p-exceptional on $V$ and is not transitive on $V^{\sharp}$. Then $L_{2}(11) \triangleleft G<G L_{5}(3)$, with orbit sizes $1,22,110,110$. 
As in previous sections, for the proof it is useful to know that the group $L$ is generated by any two of its Sylow $p$-subgroups. For $p=2$ this is true by [21]. It is presumably true for all primes, but we do not prove this here, and just cover the following groups for which we need the result.

Lemma 11.2. Let $L$ be one of the following simple groups:

$$
\begin{aligned}
& L_{n}(r): n=2, r \leq 37 ; \text { or } n=3, r \leq 5 ; \text { or } n \leq 5, r=3 ; \text { or } n \leq 8, r=2 \\
& P S p_{2 n}(r): n=2, r \leq 11 ; \text { or } n=3, r \leq 7 ; \text { or } n=4, r=5 ; \text { or } n \leq 6, r \leq 3 \\
& U_{n}(r): n=3, r \leq 5 ; \text { or } n=4, r \leq 4 ; \text { or } n=5, r \leq 3 ; \text { or } n \leq 11, r=2 \\
& \text { and } \Omega_{7}(3), \Omega_{8}^{ \pm}(2), \Omega_{10}^{ \pm}(2), F_{4}(2),{ }^{2} F_{4}(2)^{\prime},{ }^{3} D_{4}(2), G_{2}(3), G_{2}(4),{ }^{2} B_{2}(8) .
\end{aligned}
$$

Let $X$ be a group such that $L \leq X \leq \operatorname{Aut}(L)$. If $p$ is a prime dividing $|X|$, then $X$ is generated by two of its Sylow p-subgroups.

Proof This was verified computationally using Magma.

Now we embark upon the proof of Theorem 9.1. Let $G \leq \Gamma L(V)=\Gamma L_{d}(q)\left(q=p^{a}\right)$ be as in the hypothesis, with $L=\operatorname{soc}(G / Z)$ a simple group of Lie type in $p^{\prime}$-characteristic not isomorphic to an alternating group. We assume now that $G \leq G L(V)$; we shall handle the case where $G \leq \Gamma L(V)$ at the end of the proof.

Lemma 11.3. The simple group $L$ is one of the following:

$$
\begin{aligned}
& L_{2}(r)(r \leq 37), L_{3}(4), L_{3}(5), L_{4}(3), \\
& P S p_{4}(r)(r \leq 9, r \neq 8), P S p_{6}(r)(r=2,3,5), P S p_{8}(3), P S p_{10}(3), \\
& U_{3}(r)(r=3,4,5), U_{4}(2), U_{5}(2), U_{6}(2), \\
& \Omega_{7}(3), \Omega_{8}^{ \pm}(2), \\
& F_{4}(2),{ }^{2} F_{4}(2),{ }^{3} D_{4}(2), G_{2}(3), G_{2}(4),{ }^{2} B_{2}(8) .
\end{aligned}
$$

Proof By Lemma 2.4(i) we have $d=\operatorname{dim} V \leq r_{p} \log _{q}\left|G: N_{G}(P)\right|$, where $r_{p}$ is the minimal number of Sylow $p$-subgroups required to generate $O^{p^{\prime}}(G)$. We have $r_{2}=2$ by [21], while upper bounds for $r_{p}$ with $p$ odd are provided by [22, Sections 3,4,5]. On the other hand, lower bounds for $d$ are given in $[29,40]$. These, together with the above bound, imply that $L$ is one of the groups listed in Lemma 11.2. By that lemma, we have $r_{p}=2$ for these groups, and so we now have the bound

$$
d=\operatorname{dim} V \leq 2 \log _{q}\left|G: N_{G}(P)\right| .
$$

Applying this with the above-mentioned lower bounds for $d$ eliminates many of the groups in the list, and leaves just the groups in the conclusion.

Lemma 11.4. If $L=L_{2}(r)$, then $r=11$ and $L_{2}(11) \triangleleft G<G L_{5}(3)$, with orbit sizes $1,22,110,110$.

Proof Assume $L=L_{2}(r)$. By Lemma 11.3, $r \leq 37$, so certainly $\operatorname{dim} V<250$ and we can use [24] together with (8) to identify the possibilities for $L, d, q$ :

\begin{tabular}{|l|l|l|}
\hline$L$ & $d$ & $q$ \\
\hline$L_{2}(31)$ & 15 & 2 \\
$L_{2}(25)$ & 12 & 2 \\
$L_{2}(23)$ & 11 & 2,3 \\
$L_{2}(17)$ & 8 & 2 \\
$L_{2}(13)$ & 6 & 4,3 \\
& 7 & 3 \\
$L_{2}(11)$ & 10 & 2 \\
& 5 & $4,3,5$ \\
& 6 & 3 \\
$L_{2}(7)$ & 3 & 9 \\
\hline
\end{tabular}

The case where $r=11$ and $(d, q)=(5,3)$ gives the example in the conclusion; the orbit sizes are given in [7]. (Note that there are two 5 -dimensional representations of $L$ over $\mathbb{F}_{3}$, 
but they are quasiequivalent, hence have the same orbit sizes.) Also, when $r=13$ and $(d, q)=(6,3), \hat{L}=S L_{2}(13)$ is transitive on nonzero vectors (see [30, Appendix 1]), contrary to our assumption in Theorem 11.1 .

When $r=11,13,17,23$ or 25 and $(d, q)=(5,5),(6,4),(8,2),(11,2)$ or $(12,2)$, the orbit sizes are given by [7, Section 5] and [30, Appendix 2]; there is an orbit size divisible by $p$ in all cases.

Now consider the cases where $r=11,13,23$ or 31 and $(d, q)=(6,3),(7,3),(11,3)$ or $(15,2)$. For these, we observe that there is a nonzero vector fixed by a subgroup $H$ of order $11,7,11$ or 5 respectively, and $H$ generates $L$ together with any Sylow $p$-subgroup, a contradiction.

This leaves the cases where $r=7$ or 11 and $(d, q)=(3,9),(5,4)$ or $(10,2)$. For these cases a MAGMA computation shows that there is an orbit of size divisible by $p$.

Lemma 11.5. $L$ is not $L_{3}(4), L_{3}(5)$ or $L_{4}(3)$.

Proof Suppose $L$ is one of these groups. By [24] and (8), the possibilities for $L, d, q$ are:

\begin{tabular}{|l|l|l|}
\hline$L$ & $d$ & $q$ \\
\hline$L_{3}(4)$ & 4 & 9 \\
& 6 & 3,7 \\
& 8 & 5 \\
$L_{4}(3)$ & 26 & 2 \\
\hline
\end{tabular}

Consider the case where $L=L_{3}(4)$ and $(d, q)=(6,3)$. Here $L<P \Omega_{6}^{-}(3)$ and we see from [8, p.52] that $L$ is transitive on the 126 non-isotropic points in $V$, contrary to $p$-exceptionality.

For the remaining cases $(d, q)=(4,9),(8,5),(6,7),(26,2)$, there is a nonzero vector fixed by a subgroup $H$ of order 7, 7, 5 or 13 respectively, and $H$ generates $L$ together with any Sylow $p$-subgroup, a contradiction.

Lemma 11.6. $L$ is not $P S p_{2 m}(r)$ for $m \geq 2$.

Proof Suppose $L=P S p_{2 m}(r)$, so that $L$ is as in Lemma 11.3. Using [24] and (8), we see that the possibilities for $L, d, q$ are:

\begin{tabular}{|l|l|l|}
\hline$L$ & $d$ & $q$ \\
\hline$P S p_{4}(3)$ & 4 & 25 \\
& 6 & 5 \\
$P S p_{4}(5)$ & 12 & 4 \\
$P S p_{4}(7)$ & 24 & 2 \\
$P S p_{4}(9)$ & 40 & 2 \\
$P S p_{6}(3)$ & 13 & 4,7 \\
& 14 & 7 \\
$P S p_{8}(3)$ & 40 & 4 \\
$\operatorname{Sp}_{4}(4)$ & 18 & 3 \\
$S p_{6}(2)$ & 7 & $3,5,7$ \\
& 8 & $3,5,7$ \\
& 14 & 3 \\
\hline
\end{tabular}

The three cases $P S p_{4}(3)<L_{6}(5), S p_{6}(2)<L_{7}(5)$ and $S p_{6}(2)<L_{8}(7)$ were handled using a Magma computation, and in each case there is an orbit size divisible by $p$.

Now consider all the other cases in the table with $r$ odd. In each case $r^{2}$ does not divide $q^{d}-1$, so there is a subgroup $H$ fixing a nonzero vector, where $H$ is of index $r$ in a Sylow $r$ subgroup of $L$ (replacing $r$ by 3 when $L=P S p_{4}(9)$ ). However, one checks that $H$ generates $L$ with any Sylow $p$-subgroup, contradicting $p$-exceptionality (use [8], or [32] for $p=2$ ).

If $L=S p_{4}(4)$, an element of order 17 fixes a vector and generates $L$ with any Sylow 3-subgroup.

Finally let $L=S p_{6}(2)$. For the $p=3$ cases, an element of order 7 in $L$ fixes a vector and generates $L$ with any Sylow 3 -subgroup. And when $(d, q)=(7,7)$ or $(8,5)$ there are 
subgroups $\Omega_{6}^{-}(2)$ or $U_{3}(3)$, respectively, fixing a vector, and these generate $L$ with any Sylow $p$-subgroup.

Lemma 11.7. $L$ is not $U_{n}(r)$ for $n \geq 3$.

Proof Suppose $L=U_{n}(r)$ is as in Lemma 11.3. By [24] and (8), the possibilities are:

\begin{tabular}{|l|l|l|}
\hline$L$ & $d$ & $q$ \\
\hline$U_{3}(3)$ & 6 & 2 \\
& 14 & 2 \\
$U_{3}(4)$ & 12 & 3 \\
$U_{3}(5)$ & 20 & 2 \\
$U_{5}(2)$ & 10 & $3,5,11$ \\
$U_{6}(2)$ & 21 & 3 \\
& 22 & 5 \\
\hline
\end{tabular}

The group $U_{3}(3)<L_{6}(2)$ is transitive on nonzero vectors (see [30, Appendix 1]), contrary to assumption. For the cases $(d, q)=(14,2),(20,2),(10,3),(10,11)$ and $(21,3)$, there is a subgroup $H$ or order $7,7,2^{7}, 2^{7}, 11$, respectively, fixing a vector and generating $L$ with any Sylow $p$-subgroup. In the remaining three cases with $(d, q)=(12,3),(10,5)$ and $(22,5)$, a MAGMA computation shows that there is an orbit of size divisible by $p$.

Lemma 11.8. $L$ is not an orthogonal group in Lemma 11.3.

Proof Suppose $L=\Omega_{7}(3)$ or $\Omega_{8}^{ \pm}(2)$. Using [24] and (8), we see that $L=\Omega_{8}^{+}(2), d=8$ and $q=3,5$ or 7 . Here $\hat{L}=2 . L$ and these 8 -dimensional representations arise from the action of the Weyl group of $E_{8}$ on the root lattice. This is transitive on the 240 root vectors, and hence there is an orbit of size 120 on 1 -spaces. Hence $\hat{L}$ is not $p$-exceptional for $p=3,5$. For $p=7$, the total number of 1 -spaces in $V$ is not divisible by 3 , so there is a 1 -space fixed by a Sylow 3 -subgroup of $L$, which generates $L$ with any Sylow 7-subgroup of $L$.

Lemma 11.9. $L$ is not an exceptional group in Lemma 11.3.

Proof Suppose $L$ is an exceptional group. By [24] and (8), the possibilities are:

\begin{tabular}{|l|l|l|}
\hline$L$ & $d$ & $q$ \\
\hline$G_{2}(3)$ & 14 & 2 \\
$G_{2}(4)$ & 12 & $3,5,7$ \\
${ }^{2} B_{2}(8)$ & 8 & 5 \\
\hline
\end{tabular}

For $(d, q)=(14,2),(12,3),(12,5),(12,7)$, there is a subgroup $H$ of $L$ of order $3^{5}, 2^{9}, 2^{10}, 2^{8}$, respectively, fixing a 1 -space, and $H$ generates $L$ with any Sylow $p$-subgroup, a contradiction. For the remaining case $(d, q)=(8,5)$, a MAGMA computation gives an orbit of size divisible by 5 .

We now complete the proof of Theorem 11.1 by handling the case where the $p$-exceptional group $G$ in the hypothesis lies in $\Gamma L(V)$ and not in $G L(V)$, where $V=V_{d}(q)$. Let $G_{0}=$ $G \cap G L(V)$. If $p$ divides $\left|G_{0}\right|$ then $G_{0}$ is $p$-exceptional, hence is given by the linear case of the theorem which we have already proved; but this implies that $G L(V)=G L_{5}(3)$, in which case $G L(V)=\Gamma L(V)$.

Hence $p$ does not divide $\left|G_{0}\right|$, and by Lemma $2.3, G$ has a $p$-exceptional normal subgroup $G_{0}\langle\sigma\rangle$ (hence also $\hat{L}\langle\sigma\rangle$ ), where $\sigma \in \Gamma L(V) \backslash G L(V)$ is a field automorphism of order $p$. Hence $q=p^{k p}$ for some integer $k$. Since $G_{0}$ is a $p^{\prime}$-group, we have $p>2$. Moreover, $\sigma$ induces an automorphism of order $p$ of the simple $p^{\prime}$-group $L$, which must be a field automorphism, so $L=L\left(r^{p}\right)$ is a group of Lie type over $\mathbb{F}_{r^{p}}$ for some $r$.

Let $\ell$ be the untwisted Lie rank of $L$. Then $d \geq \frac{1}{2}\left(r^{p \ell}-1\right)$ by [29], and also $|\hat{L}|<r^{4 p \ell^{2}}$ and $\left|C_{V}(\sigma)\right|=q^{d\left(1-\frac{1}{p}\right)}$. By Lemma 2.4(ii), $\left|V: C_{V}(\sigma)\right|<\left|\sigma^{L}\right|$, which implies

$$
q^{\frac{1}{2}\left(r^{p \ell}-1\right) \cdot\left(1-\frac{1}{p}\right)}<r^{4 p \ell^{2}}
$$


This cannot hold.

Hence the case where $G$ lies in $\Gamma L(V)$ and not in $G L(V)$ does not occur. This completes the proof of Theorem 11.1.

\section{Proof of Theorem 1}

Let $G$ be an irreducible subgroup of $G L_{n}(p)$ with $p$ prime, and suppose that $G$ acts primitively on $V_{n}(p)$. Suppose also that $G$ is $p$-exceptional, so that $p$ divides $|G|$ and every orbit of $G$ on $V$ has size coprime to $p$.

Choose $q=p^{k}$ maximal such that $G \leq \Gamma L_{d}(q) \leq G L_{n}(p)$, where $n=d k$. Write $V=$ $V_{d}(q), G_{0}=G \cap G L_{d}(q)$ and $Z=G_{0} \cap \mathbb{F}_{q}^{*}$, the group of scalar multiples of the identity in $G_{0}$. Note that $G_{0} \triangleleft G$ and $G / G_{0}$ is cyclic. Write $K=\mathbb{F}_{q}$.

If $d=1$ then $G \leq \Gamma L_{1}(q)$, as in Theorem 1(ii). So assume that $d \geq 2$.

Lemma 12.1. $G_{0}$ is absolutely irreducible on $V=V_{d}(q)$.

Proof We have $G \leq N_{G L_{n}(p)}(K)=\Gamma L_{d}(q)$. So $G_{0}=C_{H}(K) \leq G L_{d}(q)$.

Let $E=\operatorname{End}_{G}(V)=\mathbb{F}_{r} \subseteq K$, and write $q=p^{k}=r^{b}$. Viewing $V$ as $V_{b d}(r)$, it is an absolutely irreducible $\mathbb{F}_{r} G$-module. Now view $V$ as an $\mathbb{F}_{q} G_{0}$-module. Then $U:=V \otimes_{\mathbb{F}_{r}} \mathbb{F}_{q}$, as an $\mathbb{F}_{q} G_{0}$-module, is the sum of $b$ Frobenius twists of $V$. However $G / G_{0}$ is cyclic of order at most $b$, so if $V \downarrow G_{0}$ were reducible, then $U \downarrow G$ would be reducible. But $G$ is absolutely irreducible, so this is a contradiction.

Hence $V \downarrow G_{0}$ is irreducible. As $C_{\operatorname{End}(V)}\left(G_{0}\right)$ is a field extension of $K$, the choice of $K$ implies that $C_{\operatorname{End}(V)}\left(G_{0}\right)=K$, and so $V$ is an absolutely irreducible $K G_{0}$-module.

If $G$ preserves a tensor product decomposition $V=U \otimes W$ over $\mathbb{F}_{q}$, where $\operatorname{dim} U \geq$ $\operatorname{dim} W \geq 2$ (i.e. $G \leq \operatorname{Stab}_{\Gamma L(V)}(U \otimes W)$ ), then Theorems 4.1 and 4.2 (together with Lemma 2.3) give a contradiction. So assume that $G$ does not preserve a nontrivial tensor decomposition of $V$.

Lemma 12.2. Let $N$ be a normal subgroup of $G$ such that $N \leq G_{0}$ and $N \not Z Z$. Then $V \downarrow N$ is absolutely irreducible.

Proof By Clifford's Theorem $V \downarrow N$ is a direct sum of homogeneous components; these are permuted by $G$, and hence by the primitivity of $G, V \downarrow N$ is homogeneous. Say $V \downarrow N \cong W \oplus \cdots \oplus W$, a direct sum of $k$ copies of an irreducible $K N$-module $W$. Let $K_{0}=C_{\operatorname{End}(W)}(N)$, a field extension of $K$. By the first few lines of the proof of $[1,5.7], K_{0}^{*}$ can be identified with $Z\left(C_{G L(V)}(N)\right)$ and $G \leq N_{\Gamma L(V)}\left(K_{0}\right)$, so $K_{0}=K$ by choice of $K$. Hence $W$ is an absolutely irreducible $K N$-module. At this point $[1,3.13]$ shows that there is a $K$-space $A$ such that $V$ can be identified with $W \otimes A$ in such a way that $N \leq G L(W) \otimes 1_{A}$, $G_{0} \leq G L(W) \otimes G L(A)$ and $G \leq N_{\Gamma L(V)}(G L(W) \otimes G L(A))$. By our assumption that $G$ preserves no nontrivial tensor decomposition, this implies that $W=V$, completing the proof.

Now let $S$ be the socle of $G / Z$, and write $S=M_{1} \times \cdots \times M_{k}$ where each $M_{i}$ is a minimal normal subgroup of $G / Z$. Let $R$ be the full preimage of $S$ in $G$, and $P_{i}$ the preimage of $M_{i}$, so that $R=P_{1} \ldots P_{k}$, a commuting product. If some $P_{i}$, say $P_{k}$, is not contained in $G_{0}$, then $M_{k}$ is generated by a field automorphism $\phi$ of prime order, and so $G / Z \leq C_{P \Gamma L(V)}(\phi)=P G L_{d}\left(q_{0}\right)\langle\psi\rangle$, where $\psi$ generates the Galois group of $\mathbb{F}_{q} / \mathbb{F}_{p}$ and $\mathbb{F}_{q_{0}}$ is a proper subfield of $\mathbb{F}_{q}$. This contradicts Theorem 6.1 .

Hence $R=P_{1} \ldots P_{k} \leq G_{0}$. As $P_{1} \triangleleft G$, Lemma 12.2 implies that $V \downarrow P_{1}$ is absolutely irreducible, hence $C_{G_{0}}\left(P_{1}\right)=Z$. It follows that $k=1$ and $R=P_{1}$. Also $G$ is not realised (modulo scalars) over a proper subfield of $\mathbb{F}_{q}$, by Theorem 6.1.

Suppose first that $M_{1}=R / Z$ is an elementary abelian $r$-group for some prime $r$, and replace $R$ by a minimal preimage of $M_{1}$ in $G$. By Lemma 12.2 and since $d \geq 2$, any maximal 
abelian characteristic subgroup of $R$ must be contained in $Z$. Hence $R$ is of symplectic type, and now we argue as in $[1,11.8]$ that $R$ can be taken to be as in Section 7 . Since $G$ is primitive, Theorem 7.1 now gives a contradiction.

Now suppose $M_{1}=R / Z$ is non-abelian, so $M_{1} \cong T^{l}$ for some non-abelian simple group $T$, and $R=R_{1} \ldots R_{l}$ where $R_{i} / Z \cong T$ and the factors are permuted transitively by $G$. If $l>1$ then $[1,3.16,3.17]$ implies that $R$ preserves a tensor decomposition $V=V_{1} \otimes \cdots \otimes V_{l}$ with $\operatorname{dim} V_{i}$ independent of $i$, and $G \leq N_{\Gamma L(V)}\left(\otimes G L\left(V_{i}\right)\right)$; then Theorems 5.1 and 5.2 give a contradiction.

It remains to consider the case where $l=1$, so that $M_{1}=\operatorname{Soc}(G / Z)$ is simple. Then $G / Z$ is almost simple, and has socle absolutely irreducible on $V$ and not realisable over a proper subfield of $\mathbb{F}_{q}$. In other words $G$ is in the class $\mathcal{C}_{9}$, and so $G$ is given by the results in Sections 8-11.

This completes the proof of Theorem 1.

\section{Deduction of Theorems 4, 5 And 6}

Proof of Theorem 4 Let $G \leq G L_{d}(p)=G L(V)$ be irreducible and $p$-exceptional, and let $G_{0}=O^{p^{\prime}}(G)$. Then $G_{0}$ is also $p$-exceptional by Lemma 2.1. By Clifford's Theorem, $V \downarrow G_{0}=V_{1} \oplus \cdots \oplus V_{t}$, where the $V_{i}$ are irreducible $G_{0}$-modules, conjugate under $G$. Note that $O^{p^{\prime}}\left(G_{0}\right)=G_{0}$ and $p$ divides $\left|G_{0}^{V_{1}}\right|$, so $G_{0}^{V_{1}}$ is $p$-exceptional. If $G_{0}^{V_{1}}$ is primitive, it is given by Theorem 1 , and if it is imprimitive it is given by Theorem 3 .

We now claim that the $V_{i}$ are pairwise non-isomorphic $G_{0^{-}}$-modules. Suppose false, and let $W=V_{1} \oplus \cdots \oplus V_{k}$ be a homogeneous component for $G_{0}$ with $k>1$. Then $G_{0}^{W} \leq$ $G L\left(V_{1}\right) \otimes 1 \leq G L\left(V_{1}\right) \otimes G L_{k}(p)$. Since $G_{0}$ is $p$-exceptional on $V$, it is also $p$-exceptional on $W$. We now apply Theorem 4.1, which classifies $p$-exceptional groups which preserve tensor product decompositions. From this theorem, it is clear that such a group cannot act just as scalars on one of the tensor factors, which is what $G_{0}^{W}$ does. This is a contradiction, proving the claim.

By the claim, $G$ permutes the summands $V_{i}$. Finally, the kernel $K$ of the action of $G$ on the set of summands $\left\{V_{1}, \ldots, V_{t}\right\}$ contains $G_{0}$, so $G / K$ is a $p^{\prime}$-group.

Proof of Theorem 5 Let $G$ be a finite group and let $p>2$ be a prime. Assume that $G=O^{p^{\prime}}(G)=O^{p}(G)$, that $G$ has abelian Sylow $p$-subgroups, and that $V$ is a faithful irreducible $\mathbb{F}_{p} G$-module such that every orbit of $G$ on $V$ has length coprime to $p$. These assumptions imply that $G=G^{\prime}$ and also that $p$ divides $|G|$.

Suppose first that $G$ acts primitively on $V$. Then $G$ is given by Theorem 1 . As $G$ is insoluble, it is either transitive on $V^{\sharp}$ or one of the examples in (iii) of the theorem. In the first case we refer to the list of transitive linear groups in [30, Appendix]: the only examples where $G=G^{\prime}$ and $G$ has abelian Sylow $p$-subgroups occur in conclusions (i) and (iii) of Theorem 5; and the examples in Theorem 1(iii) with $p>2$ are also in conclusion (iii).

Now suppose $G$ is imprimitive on $V$. As $G=O^{p^{\prime}}(G), G$ is given by Theorem 3. The assumptions that $p>2$ and $G$ has abelian Sylow $p$-subgroups now force $G$ to be as in conclusion (ii) of Theorem 5. This completes the proof of the corollary.

Proof of Theorem 6 Let $G \leq G L_{d}(p)$ be a $\frac{1}{2}$-transitive linear group of order divisible by $p$, and write $V=V_{d}(p)$. Let $H=V G \leq A G L_{d}(p)$, the corresponding affine permutation group acting on $V$. Since $G$ has order divisible by $p$, it does not act semiregularly on $V^{\sharp}$, and so $H$ is $\frac{3}{2}$-transitive on $V$, and hence is a primitive permutation group on $V$ by [45, 10.4]. This implies that $G$ acts irreducibly on $V$.

Since $G$ is $\frac{1}{2}$-transitive and has order divisible by $p$, it is $p$-exceptional. So if $G$ acts primitively as a linear group on $V$, then it is given by Theorem 1 . For $G=A_{c}$ or $S_{c}$ as in (iii)(a) of the theorem, the orbit sizes are given in the proof of Lemma 9.4, and we see that the only $\frac{1}{2}$-transitive example is for $c=6$ with $(d, p)=(4,2)$, in which case $G$ is transitive 
on $V^{\sharp}$. Hence $G$ is as in the conclusion of Theorem 6. Finally, if $G$ acts imprimitively on $V$ then it is given by [39, Theorem 1.1] (which determines all imprimitive $\frac{1}{2}$-transitive linear groups). The only example of order divisible by $p$ is $D_{18}<\Gamma L_{1}\left(2^{6}\right)<G L_{6}(2)$, as in (ii) of Theorem 6 .

\section{REFERENCES}

[1] M. Aschbacher, On the maximal subgroups of the finite classical groups, Invent. Math. 76 (1984), $469-514$.

[2] J. Bamberg, M. Giudici, M.W. Liebeck, C.E. Praeger and J. Saxl, The classification of almost simple $\frac{3}{2}$-transitive groups, Trans. Amer. Math. Soc., to appear.

[3] J. van Bon, A.A. Ivanov and J. Saxl, Affine distance-transitive graphs with sporadic stabilizer, Europ. J. Comb. 20 (1999), 163-177.

[4] W. Bosma, J. Cannon and C. Playoust, The Magma algebra system I: The user language, J. Symbolic Comput. 24 (1997) 235-265.

[5] P.J. Cameron, P.M. Neumann and J. Saxl, On groups with no regular orbits on the set of subsets, Arch. Math. 43 (1984), 295-296.

[6] R.W. Carter, Finite groups of Lie type. Conjugacy classes and complex characters, Wiley, Chichester, 1993.

[7] A.M. Cohen, K. Magaard and S. Shpectorov, Affine distance-transitive graphs: the cross characteristic case, Europ. J. Comb. 20 (1999), 351-373.

[8] J. H. Conway, R. T. Curtis, S. P. Norton, R. A. Parker, and R. A. Wilson, Atlas of Finite Groups, Clarendon Press, Oxford, 1985.

[9] C.W. Curtis, Modular representations of finite groups with split ( $B, N)$-pairs, in Seminar on Algebraic Groups and Related Finite Groups, Springer Lecture Notes 131, pp.57-95, Springer, Berlin, 1970.

[10] L. E. Dickson, Linear Groups With an Exposition of the Galois Field Theory, Dover, New York, 1958.

[11] S. Dolfi, R. Guralnick, C.E. Praeger and P. Spiga, Coprime subdegrees for primitive permutation groups and completely reducible linear groups, Israel J. Math., to appear.

[12] L. Dornhoff, Group representation theory, Part B: Modular representation theory, Pure and Applied Mathematics, Vol.7, Marcel Dekker, Inc., New York, 1972.

[13] W. Feit, The representation theory of finite groups, North-Holland, Amsterdam, 1982.

[14] A. Gambini Weigel and T. S. Weigel, On the orders of primitive linear $p^{\prime}$-groups. Bull. Austral. Math. Soc. 48 (1993), 495-521.

[15] The GAP group, 'GAP - groups, algorithms, and programming', Version 4.4, 2004, http://www.gapsystem.org.

[16] D. Gluck, K. Magaard, U. Riese and P. Schmid, The solution of the $k(G V)$ problem, J. Algebra 279 (2004), 694-719.

[17] D. Gluck and T. Wolf, Defect groups and character heights in blocks of solvable groups. II, J. Algebra 87 (1984), 222-246.

[18] D. Gluck and T. Wolf, Brauer's height conjecture for p-solvable groups, Trans. Amer. Math. Soc. 282 (1984), 137-152.

[19] D. Goodwin, Regular orbits of linear groups with an application to the $k(G V)$-problem I, II, J. Algebra 227 (2000), 395-432 and 433-473.

[20] D. Gorenstein, R. Lyons and R. Solomon, The classification of the finite simple groups. Number 3. Part I. Chapter A. Almost simple K-groups. Mathematical Surveys and Monographs, 40.3. American Mathematical Society, Providence, RI, 1998.

[21] R. Guralnick, Generation of simple groups, J. Algebra 103 (1986), 381-401.

[22] R.M. Guralnick and J. Saxl, Generation of finite almost simple groups by conjugates, J. Algebra 268 (2003), 519-571.

[23] C. Hering, Transitive linear groups and linear groups which contain irreducible subgroups of prime order II, J. Algebra 93 (1985), 151-164.

[24] G. Hiss and G. Malle, Low-dimensional representations of quasi-simple groups (Corrigenda), LMS J. Comput. Math. 5 (2002), 95-126.

[25] G.D. James, On the minimal dimensions of irreducible representations of symmetric groups, Math. Proc. Camb. Phil. Soc. 94 (1983), 417-424.

[26] C. Jansen, K. Lux, R. A. Parker, and R. A. Wilson, An Atlas of Brauer Characters, Oxford University Press, Oxford, 1995.

[27] P. B. Kleidman and M. W. Liebeck, The Subgroup Structure of the Finite Classical Groups, London Math. Soc. Lecture Note Ser. no. 129, Cambridge University Press, 1990.

[28] C. Köhler and H. Pahlings, Regular orbits and the $k(G V)$-problem, in Groups and computation, III (Columbus, OH, 1999), pp.209-228, Ohio State Univ. Math. Res. Inst. Publ., 8, de Gruyter, Berlin, 2001.

[29] V. Landazuri and G.M. Seitz, On the minimal degrees of projective representations of the finite Chevalley groups, J. Algebra 32 (1974), 418-443.

[30] M.W. Liebeck, The affine permutation groups of rank three, Proc. London Math. Soc., 54 (1987), $477-516$. 
[31] M. W. Liebeck, C. E. Praeger, J. Saxl, Regular subgroups of primitive permutation groups. Mem. Amer. Math. Soc. 203 (2010), no. 952, vi+74 pp.

[32] M.W. Liebeck and J. Saxl, The primitive permutation groups of odd degree, J. London Math. Soc. 31 (1985), 250-264.

[33] G. Malle, Fast-einfache Gruppen mit langen Bahnen in absolut irreduzibler Operation, J. Algebra 300 (2006), 655-672.

[34] M. Murai, On Brauer's height zero conjecture, preprint.

[35] G. Navarro and B. Späth, A reduction theorem for the Brauer height zero conjecture, in preparation.

[36] G. Navarro and P. H. Tiep, Brauer's height zero conjecture for the 2-blocks of maximal defect, J. Reine Angew. Math. (to appear).

[37] G. Navarro and P.H. Tiep, Characters of $p^{\prime}$-degree over normal subgroups, in preparation.

[38] D. Passman, Solvable $\frac{3}{2}$-transitive group, J. Algebra 7 (1967), 192-207.

[39] D.S. Passman, Exceptional $\frac{3}{2}$-transitive permutation groups, Pacific J. Math. 29 (1969), 669-713.

[40] G.M. Seitz and A.E. Zalesskii, On the minimal degrees of projective representations of the finite Chevalley groups II, J. Algebra 158 (1993), 233-243.

[41] A. Seress, Primitive groups with no regular orbits on the set of subsets, J. London Math. Soc. 29 (1997), 697-704.

[42] P. H. Tiep and A. E. Zalesskii, Unipotent elements of finite groups of Lie type and realization fields of their complex representations, J. Algebra 271 (2004), 327-390.

[43] A. Wagner, An observation on the degrees of projective representations of the symmetric and alternating groups over an arbitrary field, Arch. Math. 29 (1977), 583-589.

[44] H.N. Ward, Representations of symplectic groups, J. Algebra 20 (1972), 182-195.

[45] H. Wielandt, Finite Permutation Groups, Academic Press, New York, 1964.

[46] R.A. Wilson, et al., A World-Wide-Web Atlas of finite group representations, http://brauer.maths.qmul.ac.uk/Atlas/v3/.

[47] H. Zassenhaus, Über endliche Fastkörper, Abh. Math. Semin. Hamb. Univ. 11 (1935), pp.187-220.

E-mail address: michael.giudici@uwa.edu.au

E-mail address: m.liebeck@imperial.ac.uk

E-mail address: Cheryl.Praeger@uwa.edu.au

E-mail address: saxl@dpmms.cam.ac.uk

E-mail address: tiep@math.arizona.edu

(Michael Giudici and Cheryl E. Praeger) Centre for the Mathematics of Symmetry and Computation, School of Mathematics and Statistics, University of Western Australia, 35 Stirling Highway, Crawley, Western Australia 6009

(Martin W. Liebeck) Department of Mathematics, Imperial College, London SW7 2BZ, England

(Jan Saxl) DPMmS, CMS, University of Cambridge, Wilberforce Road, Cambridge CB3 0WB, ENGLAND

(Pham Huu Tiep) Department of Mathematics, University of Arizona, 617 N. Santa Rita Ave., Tucson, AZ 85721-0089 